Supporting Information

\title{
Access to a Chiral Phosphine-NHC Palladium(II) Complex via the Asymmetric Hydrophosphination of Achiral Vinyl Azoles
}

\author{
Jeffery Wee Kiong Seah, Yongxin Li, Sumod A. Pullarkat, and Pak-Hing Leung* \\ Division of Chemistry \& Biological Chemistry, School of Physical \& Mathematical Sciences, \\ Nanyang Technological University, Singapore 637371, Singapore
}

Email: pakhing@ntu.edu.sg 


\section{Content Page}

A. General information $\quad$ S3

B. General synthesis of substrates $\mathbf{1 a}-\mathbf{1 h} \quad$ S4 -6

$\begin{array}{ll}\text { C. Characterisation of substrates } \mathbf{1 a}-\mathbf{1 h} & \text { S7 - 17 }\end{array}$

D. General Procedure for asymmetric hydrophosphination $\quad$ S18

E. Characterization of enantioenriched products $\mathbf{3 a}, \mathbf{b}, \mathbf{d}, \mathbf{e}, \mathbf{f}$ and $\mathbf{g} \quad$ S19- 39

F. General synthesis of complex $(S)-\mathbf{5 g} \quad S 40$

$\begin{array}{ll}\text { G. Characterization of complex }(S)-5 g & \text { S40 - } 41\end{array}$

$\begin{array}{lr}\text { H. Crystallographic information } & \text { S42 - 52 }\end{array}$

I. Complete optimizations of 1a using $(R)-\mathbf{C 3} \quad$ S53 


\section{$\underline{\text { General information }}$}

Unless otherwise stated, all reactions were carried out under a positive pressure of nitrogen using standard Schlenk techniques. Solvents were purchased from their respective companies (MeOH: Fulltime Reagent, DCM, EA: Fisher Chemicals, Toluene, n-hexane: Avantor, THF: Schedelco, Acetone: VWR Chemicals) and used as supplied. Prior to use, all solvents intended for air sensitive reactions were dried and distilled or degassed where necessary. A PSL-1820 Low Temp Pairstirrer was used for conducting low temperature reactions. Thin layer chromatography (TLC) was done using Merck silica gel 60 F254 aluminium supported plates. Flash column chromatography was performed using Merck silica gel 60. NMR spectra were obtained using Bruker AV $300\left({ }^{1} \mathrm{H}\right.$ at $300 \mathrm{MHz},{ }^{13} \mathrm{C}\left\{{ }^{1} \mathrm{H}\right\}$ at $75 \mathrm{MHz},{ }^{31} \mathrm{P}\left\{{ }^{1} \mathrm{H}\right\}$ at $\left.121 \mathrm{MHz}\right)$; AV $400\left({ }^{1} \mathrm{H}\right.$ at $400 \mathrm{MHz},{ }^{13} \mathrm{C}\left\{{ }^{1} \mathrm{H}\right\}$ at $100 \mathrm{MHz},{ }^{31} \mathrm{P}\left\{{ }^{1} \mathrm{H}\right\}$ at $\left.162 \mathrm{MHz}\right)$, AV $500\left({ }^{1} \mathrm{H}\right.$ at $500 \mathrm{MHz}$, ${ }^{13} \mathrm{C}\left\{{ }^{1} \mathrm{H}\right\}$ at $125 \mathrm{MHz},{ }^{31} \mathrm{P}\left\{{ }^{1} \mathrm{H}\right\}$ at $\left.202 \mathrm{MHz}\right)$ and $\mathrm{BBFO}\left({ }^{1} \mathrm{H}\right.$ at $400 \mathrm{MHz},{ }^{13} \mathrm{C}\left\{{ }^{1} \mathrm{H}\right\}$ at $100 \mathrm{MHz}$, ${ }^{31} \mathrm{P}\left\{{ }^{1} \mathrm{H}\right\}$ at $\left.161 \mathrm{MHz}\right)$ spectrometers. Chemical shifts were reported in ppm and referenced to an internal $\mathrm{SiMe}_{4}$ standard at $\delta 0 \mathrm{ppm}$, or to the residual proton signals of the respective deuterated solvents for ${ }^{1} \mathrm{H}$ and ${ }^{13} \mathrm{C}\left\{{ }^{1} \mathrm{H}\right\}$ NMR. Melting points were measured using an SRS Optimelt Automated Point System SRS MPA100m machine. Chiral high-performance liquid chromatography (HPLC) data were acquired using an Agilent Technologies 1200 Series HPLC machine with Daicel CHIRALPAK I-series columns. Optical rotations were measured with a JASCO P-1030 Polarimeter in the specified solvent and concentration in a $0.1 \mathrm{dm}$ cell at $25.0^{\circ} \mathrm{C}$ unless otherwise stated. 


\section{General synthesis of substrates 1a - f}

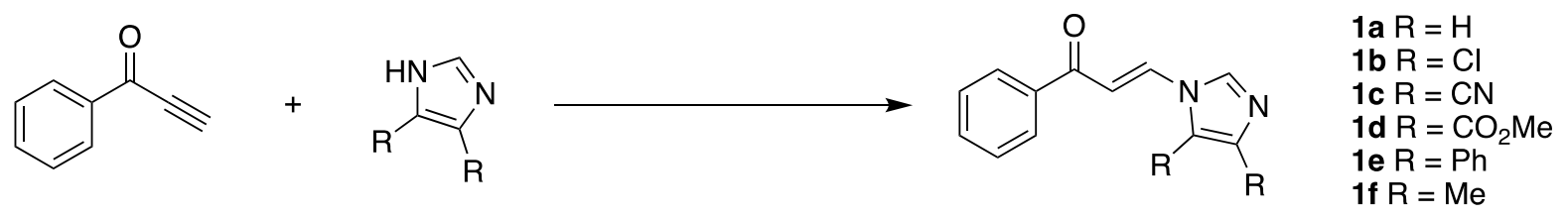

Scheme S1. Synthesis of 1a-f.

\section{Synthesis of 1a}

Adapted from literature and procedure was modified slightly. ${ }^{1}$ A $50 \mathrm{~mL}$ round-bottomed flask was charged with phenyl propargyl ketone $(0.5 \mathrm{~g}, 3.84 \mathrm{mmol})$ and THF $(30 \mathrm{~mL})$. After which, imidazole $(0.314 \mathrm{~g}, 4.61 \mathrm{mmol}, 1.2$ equiv.) was added. The reaction was heated under reflux overnight. Volatiles were removed under reduced pressure and the crude product was purified using silica gel chromatography (4 DCM:1 EA) to give pure 1a.

\section{Synthesis of $\mathbf{1 b}$}

A $50 \mathrm{~mL}$ round-bottomed flask was charged with phenyl propargyl ketone ( $0.5 \mathrm{~g}, 3.84 \mathrm{mmol})$ and THF (30 mL). After which, 4,5-dichloroimidazole $(0.631 \mathrm{~g}, 4.61 \mathrm{mmol}, 1.2$ equiv.) and triethylamine $(0.26 \mathrm{~mL}, 1.92 \mathrm{mmol}, 0.5$ equiv.) were added. The reaction was heated under reflux overnight. Volatiles were removed under reduced pressure and the crude product was purified using silica gel chromatography (4 DCM:1 EA) to give pure $\mathbf{1 b}$.

\section{Synthesis of 1c}

A $50 \mathrm{~mL}$ round-bottomed flask was charged with phenyl propargyl ketone ( $0.5 \mathrm{~g}, 3.84 \mathrm{mmol})$ and THF (30 mL). After which, 4,5-dicyano- $1 \mathrm{H}$-imidazole $(0.544 \mathrm{~g}, 4.61 \mathrm{mmol}, 1.2$ equiv.) and triethylamine $(0.26 \mathrm{~mL}, 1.92 \mathrm{mmol}, 0.5$ equiv.) were added. The reaction was heated under reflux overnight. Volatiles were removed under reduced pressure and the crude product was purified using silica gel chromatography (4 DCM:1 EA) to give pure 1c.

\section{Synthesis of 1d}

A $50 \mathrm{~mL}$ round-bottomed flask was charged with phenyl propargyl ketone (0.5 g, $3.84 \mathrm{mmol})$ and THF (30 mL). After which, dimethyl imidazole-4,5-dicarboxylate $(0.849 \mathrm{~g}, 4.61 \mathrm{mmol}$, 1.2 equiv. $)$ and triethylamine $(0.26 \mathrm{~mL}, 1.92 \mathrm{mmol}, 0.5$ equiv. $)$ were added. The reaction was heated under reflux overnight. Volatiles were removed under reduced pressure and the crude product was purified using silica gel chromatography (4 DCM:1 EA) to give pure 1d. 


\section{Synthesis of 1e}

A $50 \mathrm{~mL}$ round-bottomed flask was charged with phenyl propargyl ketone $(0.5 \mathrm{~g}, 3.84 \mathrm{mmol})$ and THF (30 mL). After which, 4,5-diphenyl-1H-imidazole (1.015 g, $4.61 \mathrm{mmol}, 1.2$ equiv.) was added. The reaction was heated under reflux overnight. Volatiles were removed under reduced pressure and the crude product was purified using silica gel chromatography (4 DCM:1 EA) to give pure $1 \mathbf{e}$.

\section{Synthesis of $1 \mathrm{f}$}

A $50 \mathrm{~mL}$ round-bottomed flask was charged with phenyl propargyl ketone $(0.5 \mathrm{~g}, 3.84 \mathrm{mmol})$ and THF (30 mL). After which, 4,5-dimethylimidazole (0.443 g, $4.61 \mathrm{mmol}, 1.2$ equiv.) was added. The reaction was heated under reflux overnight. Volatiles were removed under reduced pressure and the crude product was purified using silica gel chromatography (4 DCM:1 EA) to give pure 1f.

\section{Synthesis of substrates $1 \mathrm{~g}$}<smiles>C#CC(=O)c1ccccc1</smiles><smiles>c1ccc2[nH]cnc2c1</smiles><smiles>O=C(/C=C/n1cnc2ccccc21)c1ccccc1</smiles>

Scheme S2. Synthesis of $\mathbf{1 g}$.

A $50 \mathrm{~mL}$ round-bottomed flask was charged with phenyl propargyl ketone $(0.5 \mathrm{~g}, 3.84 \mathrm{mmol})$ and THF (30 mL). After which, benzimidazole (0.544 g, $4.61 \mathrm{mmol}, 1.2$ equiv.) and triethylamine $(0.26 \mathrm{~mL}, 1.92 \mathrm{mmol}, 0.5$ equiv. $)$ were added. The reaction was heated under reflux overnight. Volatiles were removed under reduced pressure and the crude product was purified using silica gel chromatography (4 DCM:1 EA) to give pure $\mathbf{1 g}$.

\section{Synthesis of substrates $1 \mathrm{~h}$}<smiles>C#CC(=O)c1ccccc1</smiles><smiles>c1nc[nH]n1</smiles><smiles>O=C(/C=C/n1cncn1)c1ccccc1</smiles>

$1 \mathrm{~h}$

Scheme S3. Synthesis of $\mathbf{1 h}$.

A $50 \mathrm{~mL}$ round-bottomed flask was charged with phenyl propargyl ketone (0.5 g, $3.84 \mathrm{mmol})$ and THF $(30 \mathrm{~mL})$. After which, 1,2,4-triazole $(0.381 \mathrm{~g}, 4.61 \mathrm{mmol}, 1.2$ equiv.) and 
triethylamine $(0.26 \mathrm{~mL}, 1.92 \mathrm{mmol}, 0.5$ equiv. $)$ were added. The reaction was heated under reflux overnight. Volatiles were removed under reduced pressure and the crude product was purified using silica gel chromatography (4 DCM:1 EA) to give pure $\mathbf{1 h}$. 


\section{Characterization of substrates 1a $-\mathbf{h}$}<smiles>O=C(/C=C/n1ccnc1)c1ccccc1</smiles>

Figure S1. Structure of 1 a

1a. Pale yellow solid. Yield: $0.457 \mathrm{~g}, 2.30 \mathrm{mmol}, 60 \% .{ }^{1} \mathrm{H} \mathrm{NMR}\left(\mathrm{CD}_{2} \mathrm{Cl}_{2}, 500 \mathrm{MHz}\right): \delta 7.17$, $7.21(\mathrm{~m}, 2 \mathrm{H}), 7.41(\mathrm{~s}, 1 \mathrm{H}), 7.53\left(\mathrm{t}, 2 \mathrm{H}, \mathrm{J}_{\mathrm{HH}}=7.4 \mathrm{~Hz}\right), 7.63\left(\mathrm{t}, 1 \mathrm{H}, \mathrm{J}_{\mathrm{HH}} 7.0=\mathrm{Hz}\right), 7.86(\mathrm{~s}, 1 \mathrm{H})$, $7.99\left(\mathrm{~d}, 2 \mathrm{H}, \mathrm{J}_{\mathrm{HH}}=7.4 \mathrm{~Hz}\right), 8.04\left(\mathrm{~d}, 1 \mathrm{H}, \mathrm{J}_{\mathrm{HH}}=14 \mathrm{~Hz}\right) ;{ }^{13} \mathrm{C}\left\{{ }^{1} \mathrm{H}\right\} \mathrm{NMR}\left(\mathrm{CDCl}_{3}, 75 \mathrm{MHz}\right): \delta 110.3$ (s), 116.3 (s), 128.3 (s), 128.8 (s), 132.0 (s), 133.4 (s), 136.7 (s), 137.6 (s), 138.4 (s), 188.9 (s); HRMS (+ESI) m/z: $(\mathrm{M}+\mathrm{H})+$ calcd for $\mathrm{C}_{12} \mathrm{H}_{11} \mathrm{~N}_{2} \mathrm{O}$, 199.0871; found, 199.0871. Anal. Calcd for $\mathrm{C}_{12} \mathrm{H}_{10} \mathrm{~N}_{2} \mathrm{O}$ : C, 72.71; H, 5.09; N, 14.13. Found: C, 72.53; H, 4.90 ; N, 14.23.

1H (CD2Cl2, $500 \mathrm{MHz}) \mathrm{H} 2$ substrate

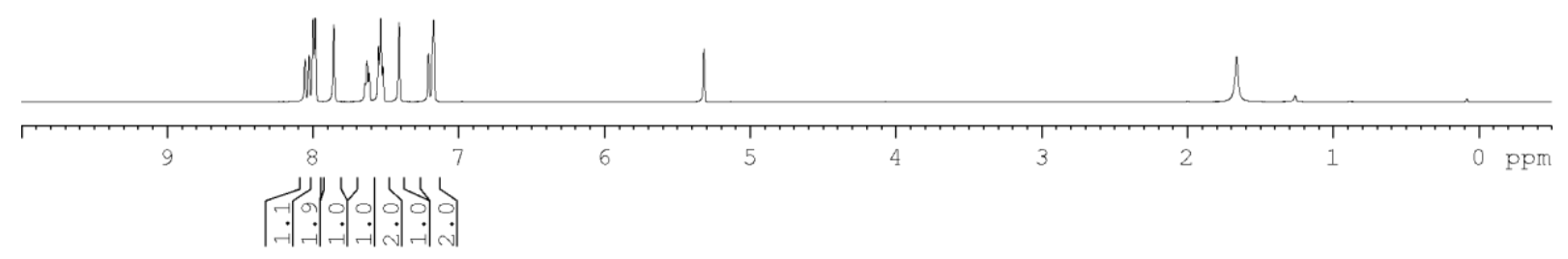

Figure S2. ${ }^{1} \mathrm{H}$ NMR spectrum of $\mathbf{1 a}$ 

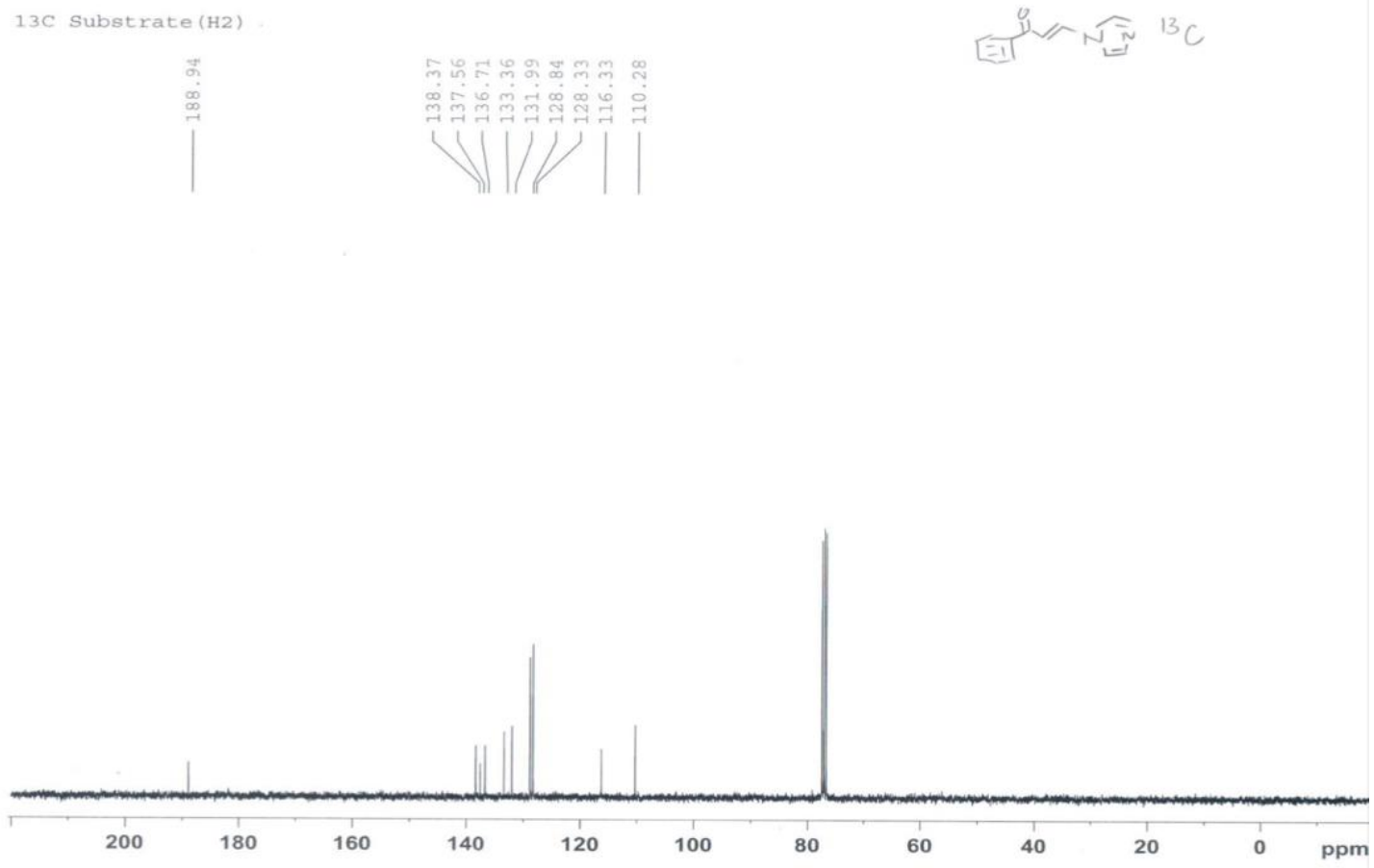

Figure S3. ${ }^{13} \mathrm{C}$ NMR spectrum of 1a<smiles>O=C(/C=C/n1cnc(Cl)c1Cl)c1ccccc1</smiles>

Figure S4. Structure of $\mathbf{1 b}$

1b. Off-white solid. Yield: $0.563 \mathrm{~g}, 2.11 \mathrm{mmol}, 55 \% . \% .{ }^{1} \mathrm{H} \mathrm{NMR}\left(\mathrm{CD}_{2} \mathrm{Cl}_{2}, 500 \mathrm{MHz}\right): \delta 7.44$ $\left(\mathrm{d}, 1 \mathrm{H}, \mathrm{J}_{\mathrm{HH}}=14 \mathrm{~Hz}\right), 7.55\left(\mathrm{t}, 2 \mathrm{H}, \mathrm{J}_{\mathrm{HH}}=7.3 \mathrm{~Hz}\right), 7.66\left(\mathrm{t}, 1 \mathrm{H}, \mathrm{J}_{\mathrm{HH}}=7.1 \mathrm{~Hz}\right), 7.92-7.96(\mathrm{~m}, 2 \mathrm{H})$, $8.01\left(\mathrm{~d}, 2 \mathrm{H}, \mathrm{J}_{\mathrm{HH}}=7.4 \mathrm{~Hz}\right) ;{ }^{13} \mathrm{C}\left\{{ }^{1} \mathrm{H}\right\} \mathrm{NMR}\left(\mathrm{CD}_{2} \mathrm{Cl}_{2}, 125 \mathrm{MHz}\right): \delta 113.1(\mathrm{~s}), 114.6(\mathrm{~s}), 128.7$ (s), 129.1 (s), 129.3 (s), 133.6 (s), 133.8 (s), 134.0 (s), 137.6 (s), 188.4 (s); HRMS (+ESI) m/z: $(\mathrm{M}+\mathrm{H})+$ calcd for $\mathrm{C}_{12} \mathrm{H}_{9} \mathrm{~N}_{2} \mathrm{OCl}_{2}, 267.0092$; found, 267.0093. Anal. Calcd for $\mathrm{C}_{12} \mathrm{H}_{8} \mathrm{~N}_{2} \mathrm{OCl}_{2}$ : C, 53.96; H, 3.02; N, 10.49. Found: C, 53.66; H, 2.45 ; N, 10.38. 


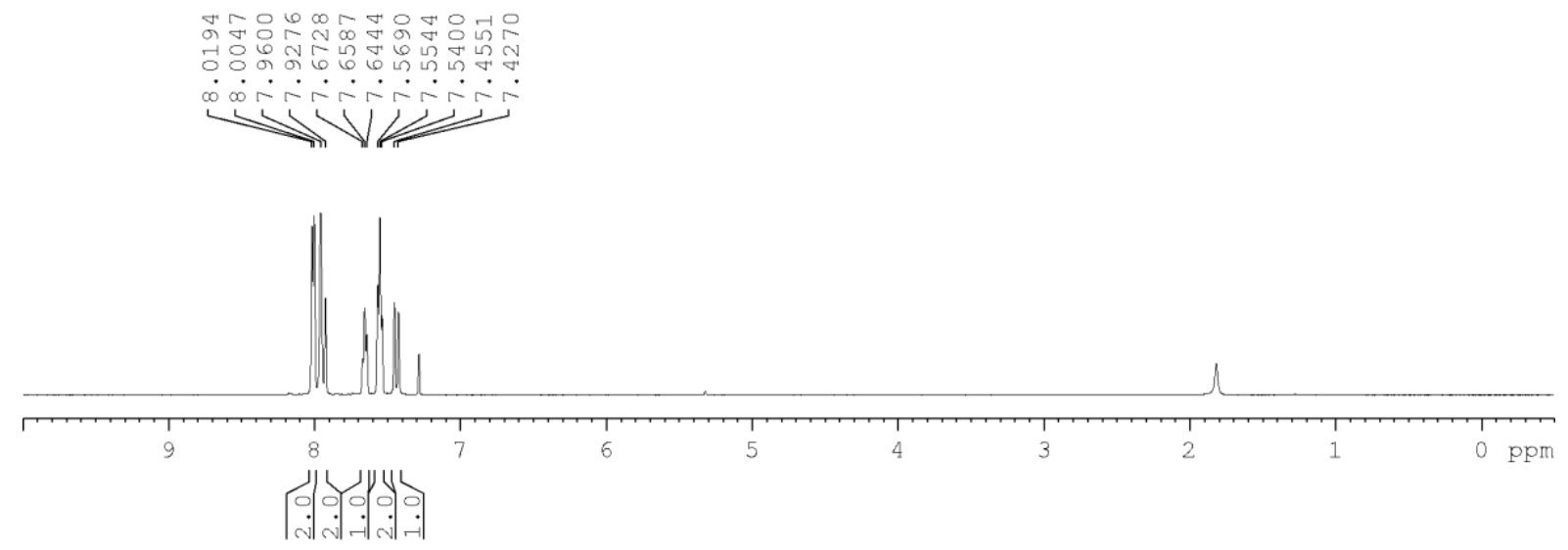

Figure S5. ${ }^{1} \mathrm{H}$ NMR spectrum of $\mathbf{1 b}$

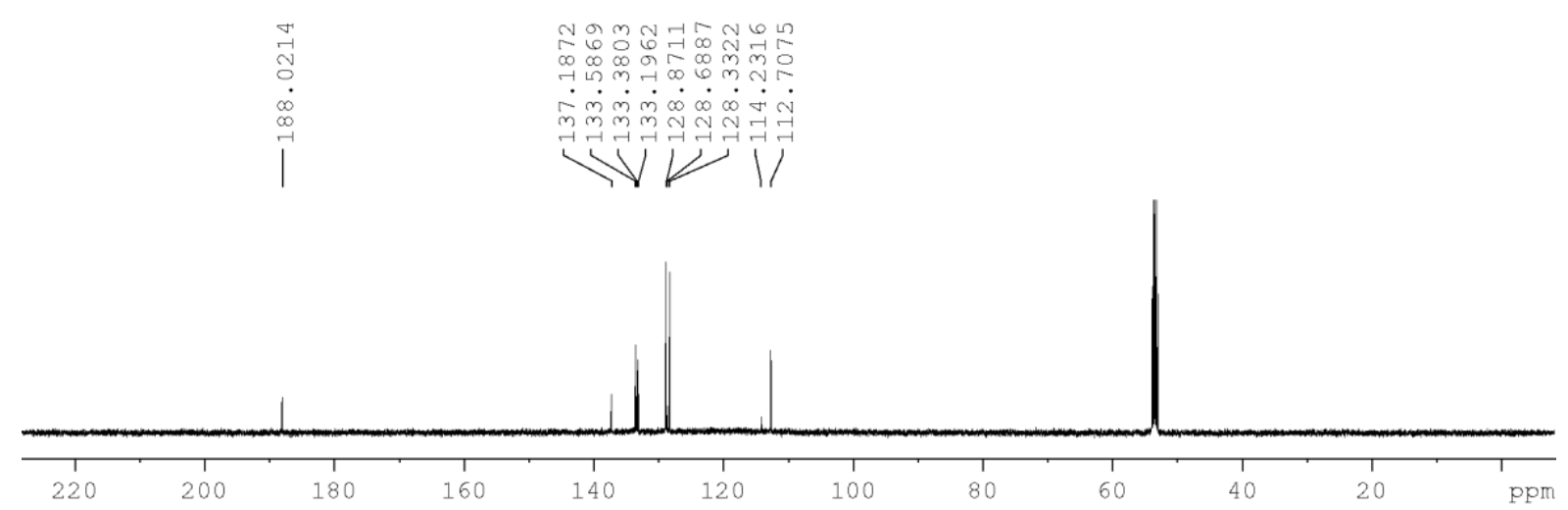

Figure S6. ${ }^{13} \mathrm{C}$ NMR spectrum of $\mathbf{1 b}$<smiles>N#Cc1ncn(/C=C/C(=O)c2ccccc2)c1C#N</smiles>

Figure S7. Structure of 1c

1c. Yellow solid. Yield: $0.315 \mathrm{~g}, 1.27 \mathrm{mmol}, 33 \% .{ }^{1} \mathrm{H} \mathrm{NMR}\left(\mathrm{CD}_{2} \mathrm{Cl}_{2}, 500 \mathrm{MHz}\right): \delta 7.58(\mathrm{t}, 2 \mathrm{H}$, $\left.\mathrm{J}_{\mathrm{HH}}=7.6 \mathrm{~Hz}\right), 7.69-7.76(\mathrm{~m}, 2 \mathrm{H}), 7.96-8.02(\mathrm{~m}, 3 \mathrm{H}), 8.14(\mathrm{~s}, 1 \mathrm{H}) ;{ }^{13} \mathrm{C}\left\{{ }^{1} \mathrm{H}\right\} \mathrm{NMR}\left(\mathrm{CDCl}_{3}\right.$, $75 \mathrm{MHz}$ ): $\delta 107.8$ (s), 110.7 (s), 116.9 (s), 125.6 (s), 128.6 (s), 129.2 (s), 132.5 (s), 134.5 (s), 136.3 (s), 140.4 (s), 140.7 (s), 186.9 (s); HRMS (+ESI) m/z: (M + H)+ calcd for $\mathrm{C}_{14} \mathrm{H}_{9} \mathrm{~N}_{4} \mathrm{O}$, 
249.0776; found, 249.0775. Anal. Calcd for $\mathrm{C}_{14} \mathrm{H}_{8} \mathrm{~N}_{4} \mathrm{O}: \mathrm{C}, 67.74 ; \mathrm{H}, 3.25 ; \mathrm{N}, 22.57$. Found: C, $67.19 ; \mathrm{H}, 3.02 ; \mathrm{N}, 22.29$.

1H (CD2C12, $500 \mathrm{MHz})$ CN2 substrate

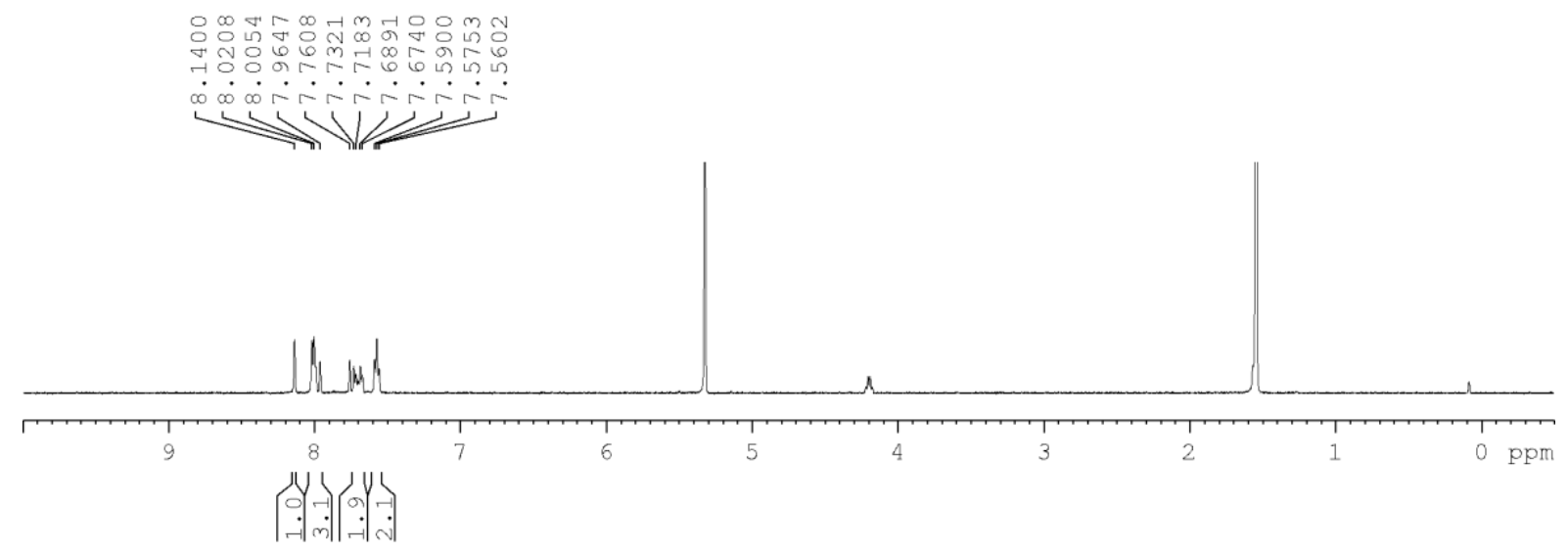

Figure S8. ${ }^{1} \mathrm{H}$ NMR spectrum of $1 \mathrm{c}$

$13 \mathrm{C}\left(\mathrm{CDCl}_{3}, 75 \mathrm{MHz}\right) \mathrm{CN} 2$ substrate

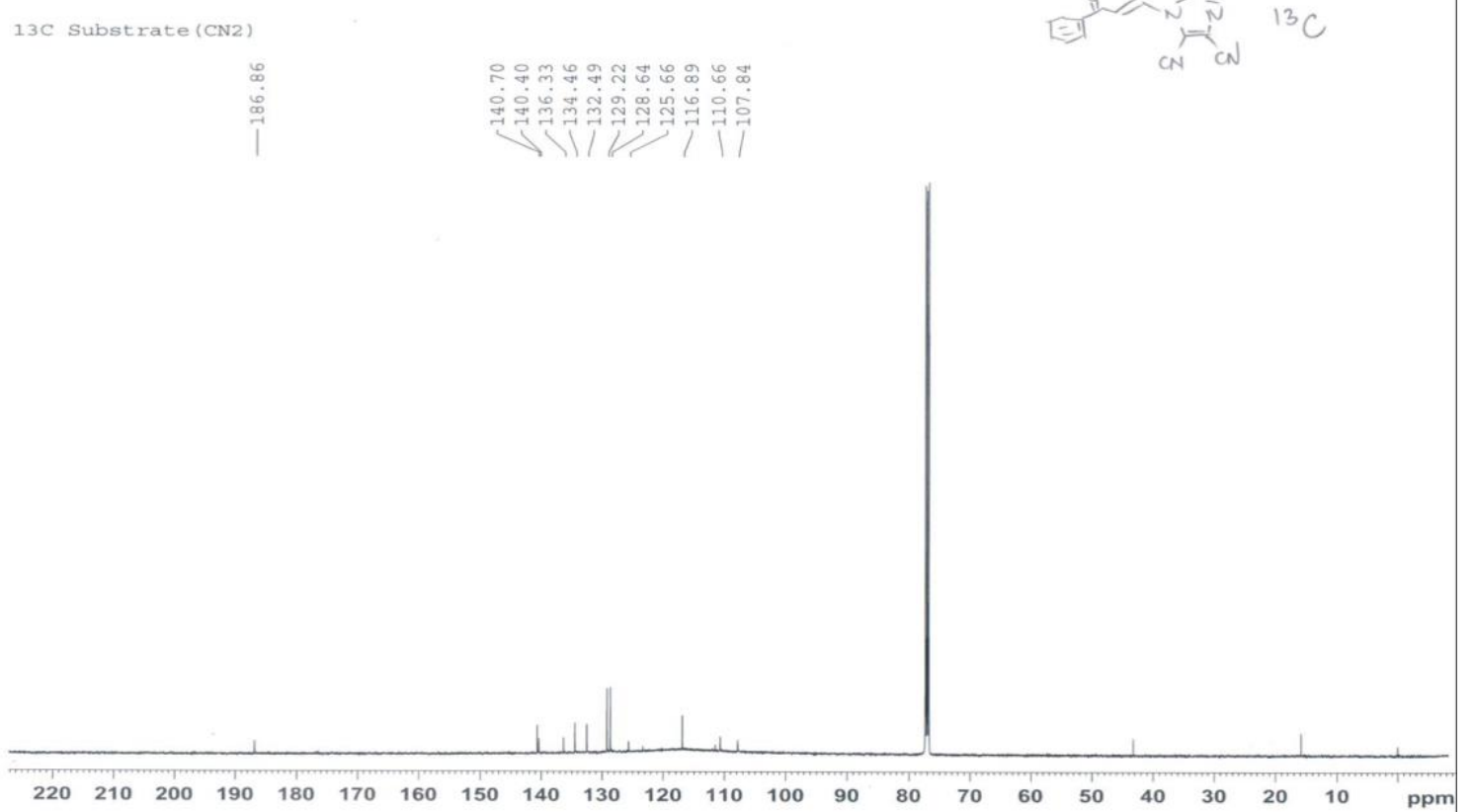

Figure S9. ${ }^{13} \mathrm{C}$ NMR spectrum of $1 \mathrm{c}$ 


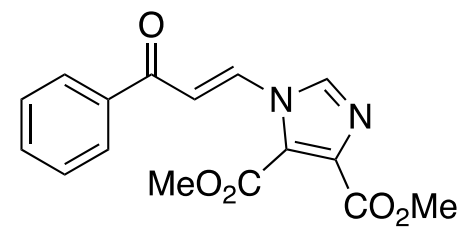

Figure S10. Structure of 1d

1d. White solid. Yield: $0.761 \mathrm{~g}, 2.42 \mathrm{mmol}, 63 \% .{ }^{1} \mathrm{H} \mathrm{NMR}\left(\mathrm{CD}_{2} \mathrm{Cl}_{2}, 500 \mathrm{MHz}\right): \delta 3.90(\mathrm{~s}, 3 \mathrm{H}$, $\mathrm{CO}_{2} \mathrm{Me}$ ), $3.95\left(\mathrm{~s}, 3 \mathrm{H}, \mathrm{CO}_{2} \mathrm{Me}\right), 7.33\left(\mathrm{~d}, 1 \mathrm{H}, \mathrm{J}_{\mathrm{HH}}=14.4 \mathrm{~Hz}\right), 7.55\left(\mathrm{t}, 2 \mathrm{H}, \mathrm{J}_{\mathrm{HH}}=7.4 \mathrm{~Hz}\right), 7.64(\mathrm{~d}$, $\left.1 \mathrm{H}, \mathrm{J}_{\mathrm{HH}}=6.8 \mathrm{~Hz}\right), 7.98\left(\mathrm{~d}, 2 \mathrm{H}, \mathrm{J}_{\mathrm{HH}}=7.5 \mathrm{~Hz}\right), 8.10(\mathrm{~s}, 1 \mathrm{H}), 8.29\left(\mathrm{~d}, 1 \mathrm{H}, \mathrm{J}_{\mathrm{HH}}=14.5 \mathrm{~Hz}\right) ;{ }^{13} \mathrm{C}\left\{{ }^{1} \mathrm{H}\right\}$ NMR ( $\left.\mathrm{CDCl}_{3}, 75 \mathrm{MHz}\right): \delta 52.6(\mathrm{~s}), 53.2(\mathrm{~s}), 115.7$ (s), 125.2 (s), 128.5 (s), $128.9(\mathrm{~s}), 133.7$ (s), 135.5 (s), 136.7 (s), 137.0 (s), 137.9 (s), 159.7 (s), 162.0 (s), 188.3 (s); HRMS (+ESI) m/z: (M $+\mathrm{H})+$ calcd for $\mathrm{C}_{16} \mathrm{H}_{15} \mathrm{~N}_{2} \mathrm{O}_{5}, 315.0981$; found, 315.0981. Anal. Calcd for $\mathrm{C}_{16} \mathrm{H}_{14} \mathrm{~N}_{2} \mathrm{O}_{5}$ : C, 61.14; H, 4.49; N, 8.91. Found: C, 61.09; H, 4.00 ; N, 8.88.

1H (CD2Cl2, $500 \mathrm{MHz})$ (CO2Me) 2 substrate

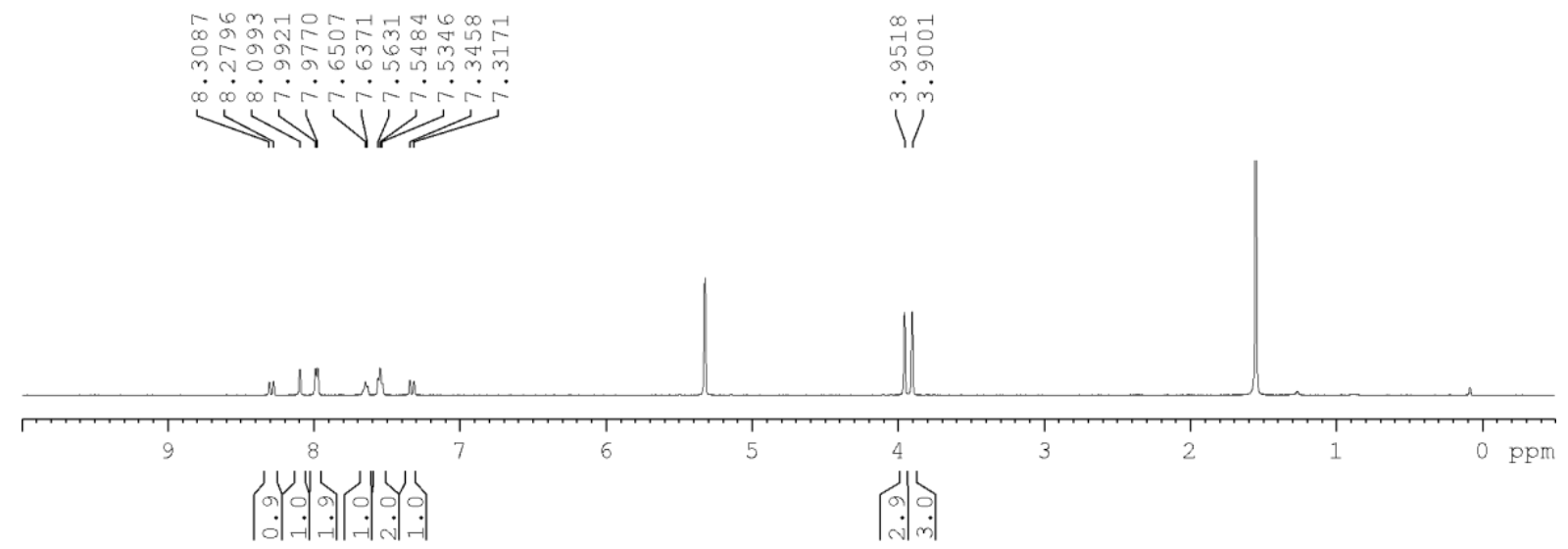

Figure S11. ${ }^{1} \mathrm{H}$ NMR spectrum of $1 d$ 


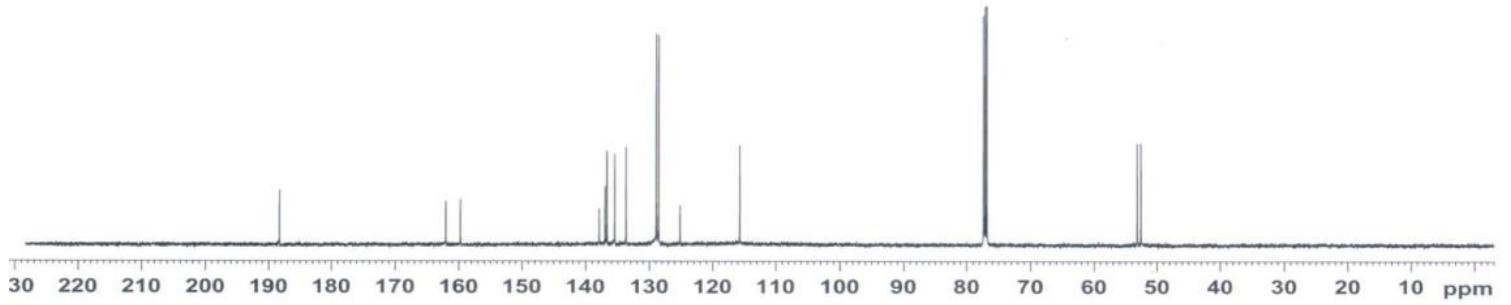

Figure S12. ${ }^{13} \mathrm{C}$ NMR spectrum of $\mathbf{1 d}$<smiles>O=C(/C=C/n1cnc(-c2ccccc2)c1-c1ccccc1)c1ccccc1</smiles>

Figure S13. Structure of $1 \mathrm{e}$

1e. White solid. Yield: $0.673 \mathrm{~g}, 1.92 \mathrm{mmol}, 50 \% .{ }^{1} \mathrm{H} \mathrm{NMR}\left(\mathrm{CD}_{2} \mathrm{Cl}_{2}, 500 \mathrm{MHz}\right): \delta 7.10(\mathrm{~d}, 1 \mathrm{H}$, $\left.\mathrm{J}_{\mathrm{HH}}=14 \mathrm{~Hz}\right), 7.11-7.42(\mathrm{~m}, 3 \mathrm{H}), 7.48-7.61(\mathrm{~m}, 10 \mathrm{H}), 7.70\left(\mathrm{~d}, 1 \mathrm{H}, \mathrm{J}_{\mathrm{HH}}=14 \mathrm{~Hz}\right), 7.86(\mathrm{~d}, 2 \mathrm{H}$, $\left.\mathrm{J}_{\mathrm{HH}}=7.6 \mathrm{~Hz}\right), 8.17(\mathrm{~s}, 1 \mathrm{H}) ;{ }^{13} \mathrm{C}\left\{{ }^{1} \mathrm{H}\right\} \mathrm{NMR}\left(\mathrm{CD}_{2} \mathrm{Cl}_{2}, 125 \mathrm{MHz}\right): \delta 110.8(\mathrm{~s}), 126.8(\mathrm{~s}), 127.1$ (s), 128.1 (s), 128.1 (s), 128.1 (s), 128.7 (s), 129.3 (s). 129.4 (s), 129.5 (s), 131.2 (s), 133.1 (s), 133.6 (s), 135.3 (s), 135.7 (s), 137.6 (s), 139.9 (s), 188.5 (s); HRMS (+ESI) m/z: (M + H)+ calcd for $\mathrm{C}_{24} \mathrm{H}_{19} \mathrm{~N}_{2} \mathrm{O}, 351.1491$; found, 351.1494. Anal. Calcd for $\mathrm{C}_{24} \mathrm{H}_{18} \mathrm{~N}_{2} \mathrm{O}: \mathrm{C}, 82.26 ; \mathrm{H}$, 5.18; N, 7.99. Found: C, 82.41; H, $5.33 ; \mathrm{N}, 8.10$. 


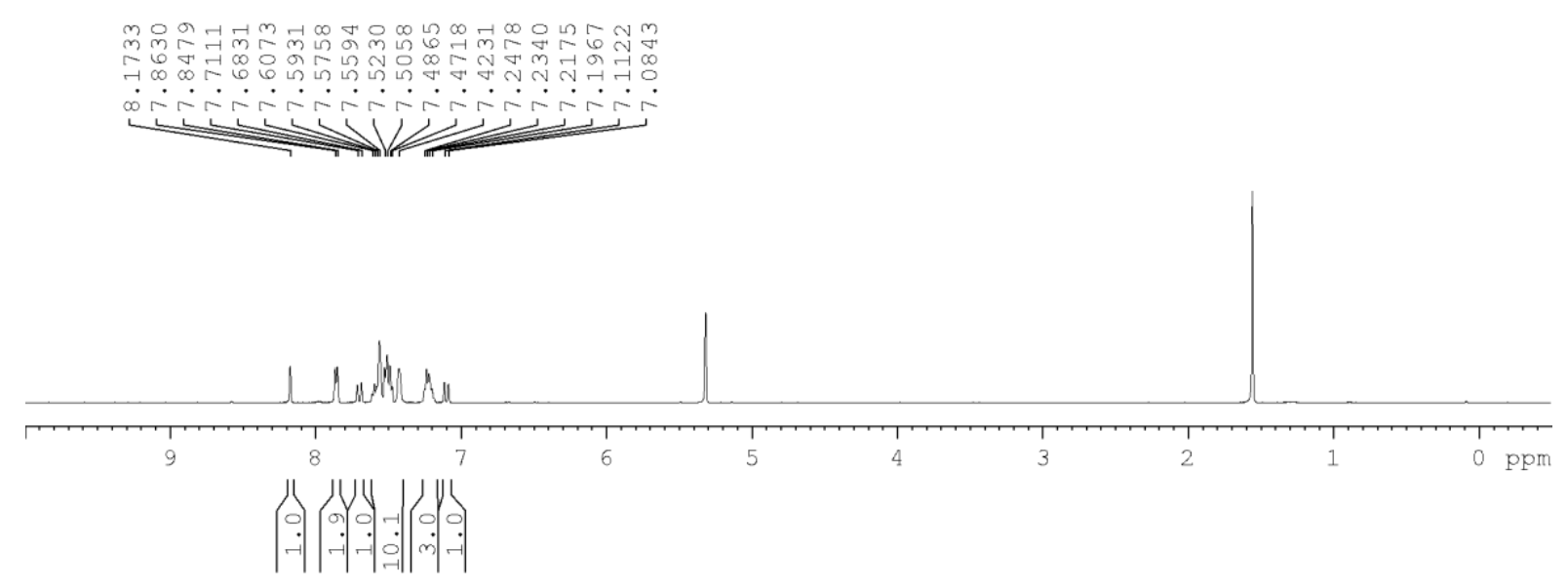

Figure S14. ${ }^{1} \mathrm{H}$ NMR spectrum of $1 \mathrm{e}$

13C (CD2C12, $500 \mathrm{MHz}$ ) substrate $\mathrm{Ph} 2$

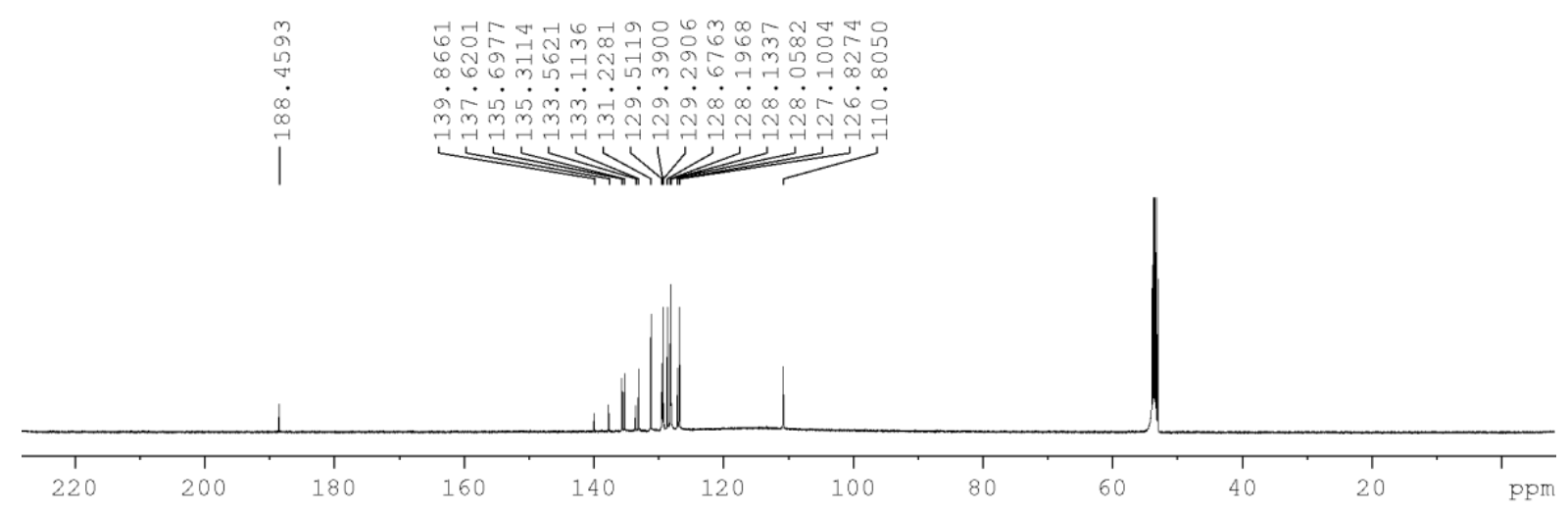

Figure S15: ${ }^{13} \mathrm{C}$ NMR spectrum of $1 \mathrm{e}$<smiles>Cc1ncn(/C=C/C(=O)c2ccccc2)c1C</smiles>

Figure S16. Structure of $\mathbf{1 f}$

1f. Yellow solid. Yield: $0.200 \mathrm{~g}, 0.883 \mathrm{mmol}, 23 \% .{ }^{1} \mathrm{H} \mathrm{NMR}\left(\mathrm{CD}_{2} \mathrm{Cl}_{2}, 500 \mathrm{MHz}\right): \delta 2.15$ (s, $3 \mathrm{H}, \mathrm{Me}), 2.28(\mathrm{~s}, 3 \mathrm{H}, \mathrm{Me}), 7.22\left(\mathrm{~d}, 1 \mathrm{H}, \mathrm{J}_{\mathrm{HH}}=14 \mathrm{~Hz}\right), 7.53\left(\mathrm{t}, 2 \mathrm{H}, \mathrm{J}_{\mathrm{HH}}=7.8 \mathrm{~Hz}\right), 7.62(\mathrm{~m}, 1 \mathrm{H})$, $7.90(\mathrm{~m}, 2 \mathrm{H}), 7.97\left(\mathrm{~d}, 2 \mathrm{H}, \mathrm{J}_{\mathrm{HH}}=7.5 \mathrm{~Hz}\right) ;{ }^{13} \mathrm{C}\left\{{ }^{1} \mathrm{H}\right\} \mathrm{NMR}\left(\mathrm{CDCl}_{3}, 75 \mathrm{MHz}\right): \delta 8.9(\mathrm{~s}), 12.6(\mathrm{~s})$, 109.1 (s), 122.8 (s), 128.3 (s), 128.8 (s), 133.2 (s), 133.5 (s), 135.9 (s), 136.4 (s), 137.8 (s), 
189.0 (s); HRMS (+ESI) m/z: $(\mathrm{M}+\mathrm{H})+$ calcd for $\mathrm{C}_{14} \mathrm{H}_{15} \mathrm{~N}_{2} \mathrm{O}, 227.1184$; found, 227.1177. Anal. Calcd for $\mathrm{C}_{14} \mathrm{H}_{14} \mathrm{~N}_{2} \mathrm{O}$ : C, 74.31; H, 6.24; N, 12.38. Found: C, 73.90; H, $6.25 ; \mathrm{N}, 12.15$.

1H (CD2C12, $500 \mathrm{MHz}) \mathrm{Me} 2$ substrate
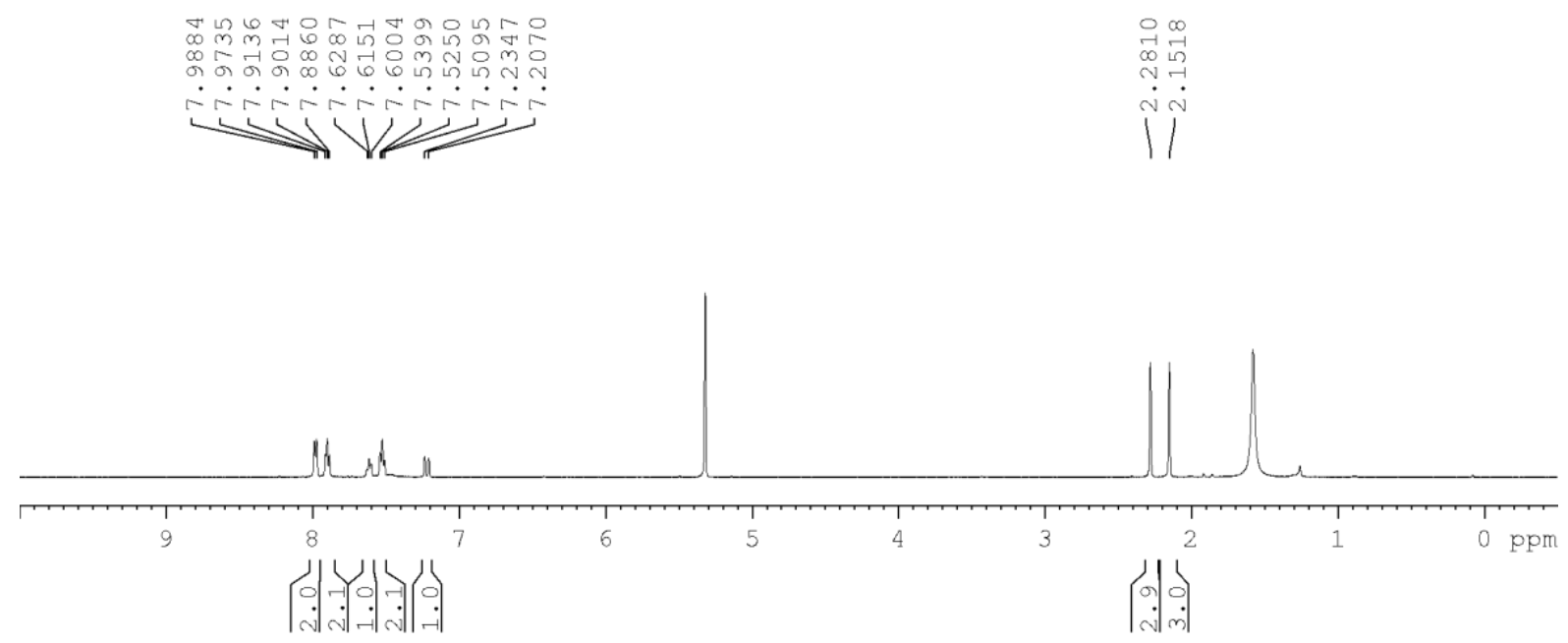

Figure S17: ${ }^{1} \mathrm{H}$ NMR spectrum of $\mathbf{1 f}$

$13 \mathrm{C}\left(\mathrm{CDCl}_{3}, 75 \mathrm{MHz}\right) \mathrm{Me} 2$ substrate

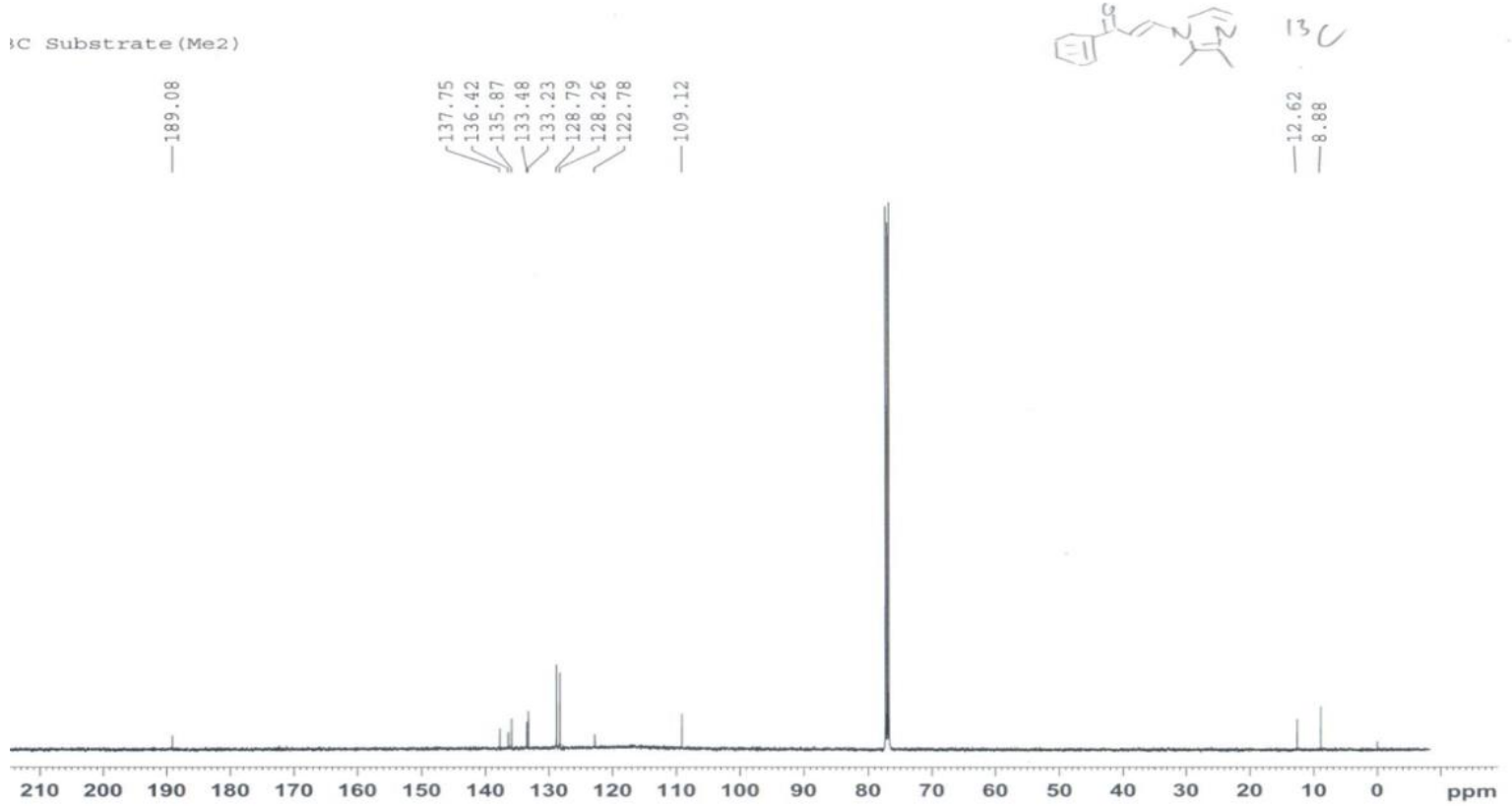

Figure S18: ${ }^{13} \mathrm{C}$ NMR spectrum of $\mathbf{1 f}$ 
<smiles>O=C(/C=C/n1cnc2ccccc21)c1ccccc1</smiles>

Figure S19. Structure of 19

1g. White solid. Yield: $0.467 \mathrm{~g}, 1.96 \mathrm{mmol}, 51 \% .{ }^{1} \mathrm{H} \mathrm{NMR}\left(\mathrm{CD}_{2} \mathrm{Cl}_{2}, 500 \mathrm{MHz}\right): \delta 7.40-7.47$ $(\mathrm{m}, 3 \mathrm{H}), 7.56\left(\mathrm{t}, 2 \mathrm{H}, \mathrm{J}_{\mathrm{HH}}=7.3 \mathrm{~Hz}\right), 7.64\left(\mathrm{t}, 1 \mathrm{H}, \mathrm{J}_{\mathrm{HH}}=6.8 \mathrm{~Hz}\right), 7.78\left(\mathrm{~d}, 1 \mathrm{H}, \mathrm{J}_{\mathrm{HH}}=8 \mathrm{~Hz}\right), 7.83$ $\left(\mathrm{d}, 1 \mathrm{H}, \mathrm{J}_{\mathrm{HH}}=8 \mathrm{~Hz}\right), 8.05\left(\mathrm{~d}, 2 \mathrm{H}, \mathrm{J}_{\mathrm{HH}}=7.4 \mathrm{~Hz}\right), 8.33\left(\mathrm{~d}, 2 \mathrm{H}, \mathrm{J}_{\mathrm{HH}}=12 \mathrm{~Hz}\right) ;{ }^{13} \mathrm{C}\left\{{ }^{1} \mathrm{H}\right\} \mathrm{NMR}$ ( $\mathrm{CDCl}_{3}, 75 \mathrm{MHz}$ ): $\delta 109.0$ (s), 111.3 (s), 121.3 (s), 124.5 (s), 125.1 (s), 128.3 (s), 128.9 (s), 132.4 (s), 133.3 (s), 135.7 (s), 137.8 (s), 141.7 (s), 144.7 (s), 189.0 (s); HRMS (+ESI) m/z: (M $+\mathrm{H})+$ calcd for $\mathrm{C}_{16} \mathrm{H}_{13} \mathrm{~N}_{2} \mathrm{O}, 249.1028$; found, 249.1036. Anal. Calcd for $\mathrm{C}_{16} \mathrm{H}_{12} \mathrm{~N}_{2} \mathrm{O}: \mathrm{C}, 77.40$; H, 4.87; N, 11.28. Found: C, 77.31; H, 4.47 ; N, 11.35.

$1 \mathrm{H}(\mathrm{CD} 2 \mathrm{Cl2}, 500 \mathrm{MHz}) \mathrm{Bn}$ substrate
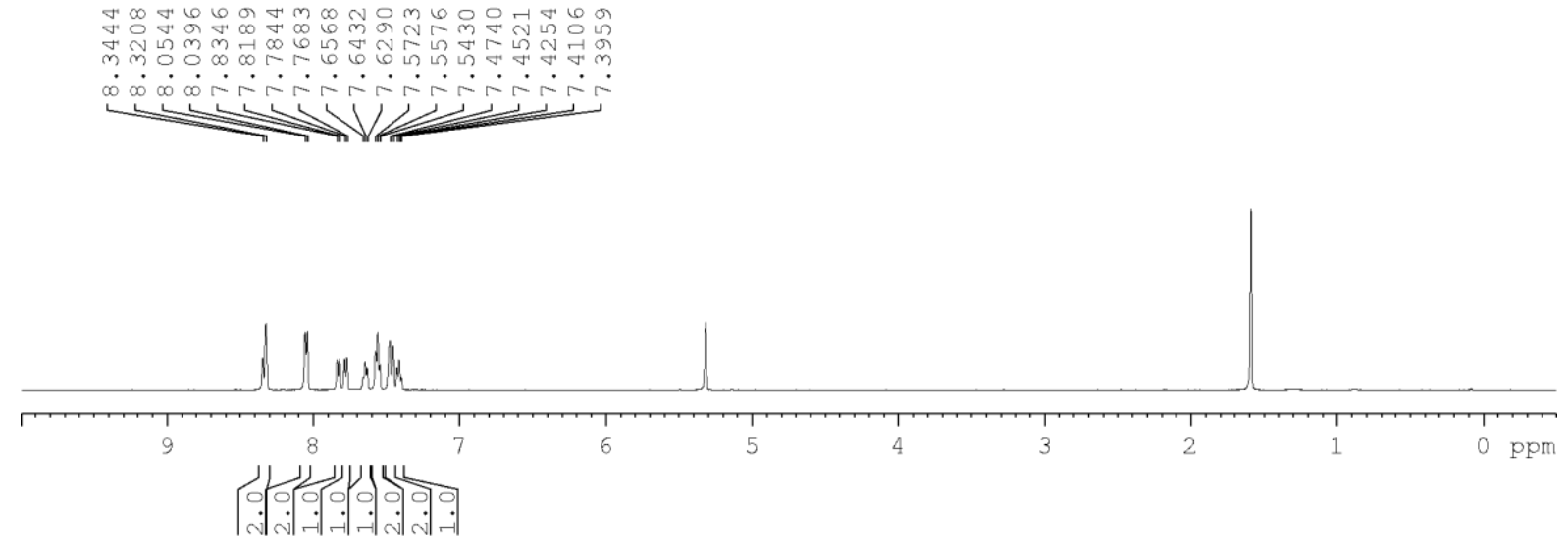

Figure S20: ${ }^{1} \mathrm{H}$ NMR spectrum of $\mathbf{1 g}$ 
$13 \mathrm{C}\left(\mathrm{CDCl}_{3}, 75 \mathrm{MHz}\right) \mathrm{Bn}$ substrate

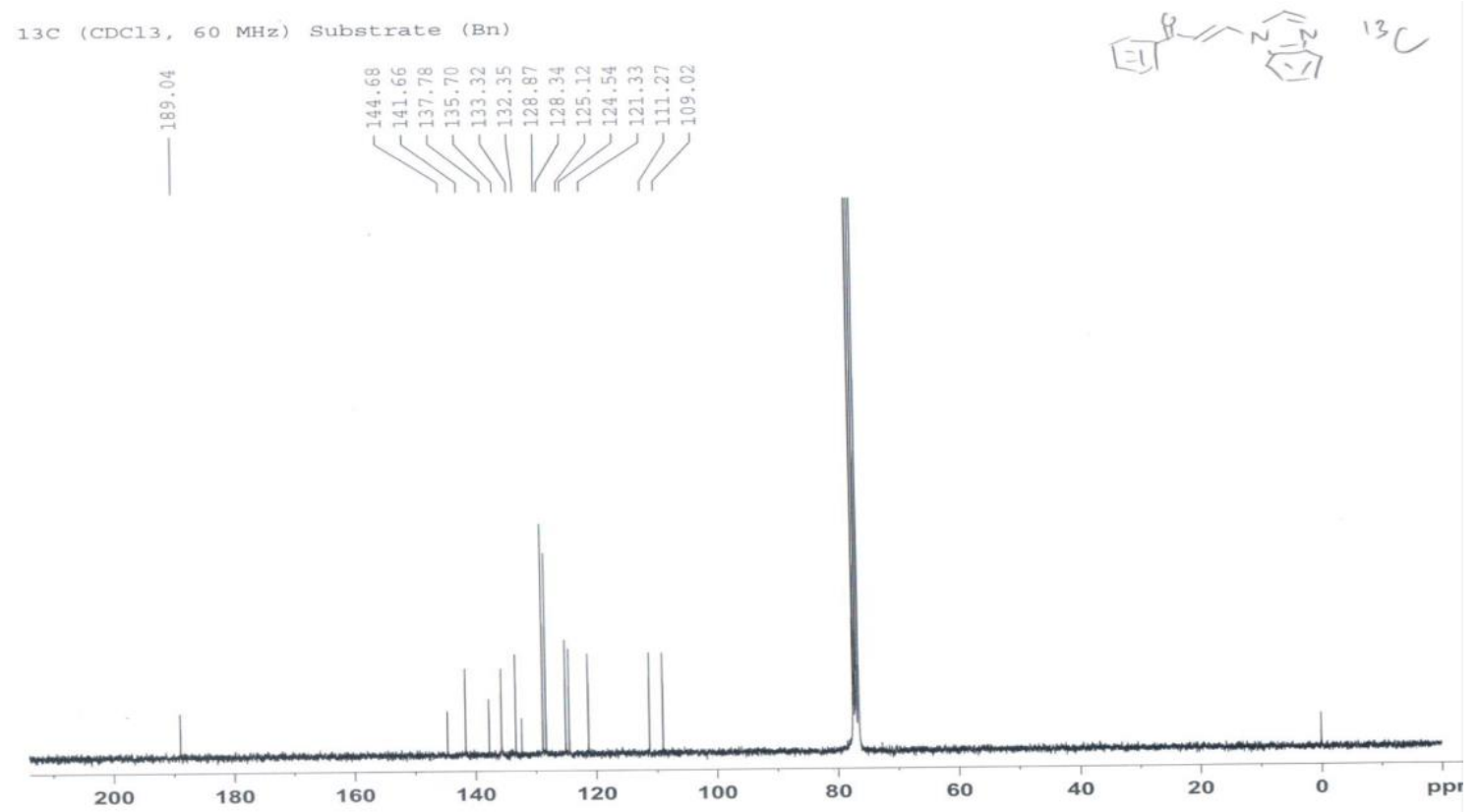

Figure S21: ${ }^{13} \mathrm{C}$ NMR spectrum of $\mathbf{1 g}$<smiles>O=C(/C=C/n1cncn1)c1ccccc1</smiles>

Figure S22. Structure of $\mathbf{1 h}$

1h. White solid. Yield: $0.275 \mathrm{~g}, 1.38 \mathrm{mmol}, 36 \% .{ }^{1} \mathrm{H}$ NMR $\left(\mathrm{CD}_{2} \mathrm{Cl}_{2}, 500 \mathrm{MHz}\right): \delta 7.54(\mathrm{t}, 2 \mathrm{H}$ $\left.\mathrm{J}_{\mathrm{HH}}=7.5 \mathrm{~Hz}\right), 7.63\left(\mathrm{t}, 1 \mathrm{H}, \mathrm{J}_{\mathrm{HH}}=7.2 \mathrm{~Hz}\right), 7.75\left(\mathrm{~d}, 1 \mathrm{H}, \mathrm{J}_{\mathrm{HH}}=13.5 \mathrm{~Hz}\right), 8.03-8.15(\mathrm{~m}, 4 \mathrm{H}), 8.43$ $(\mathrm{s}, 1 \mathrm{H}) ;{ }^{13} \mathrm{C}\left\{{ }^{1} \mathrm{H}\right\} \mathrm{NMR}\left(\mathrm{CDCl}_{3}, 75 \mathrm{MHz}\right): \delta 113.3$ (s), 128.6 (s), $128.9(\mathrm{~s}), 133.6(\mathrm{~s}), 134.8$ (s), 137.3 (s), 145.1 (s), 153.6 (s), 188.8 (s); HRMS (+ESI) m/z: (M + H)+ calcd for $\mathrm{C}_{11} \mathrm{H}_{10} \mathrm{~N}_{3} \mathrm{O}$, 200.0824; found, 200.0824. Anal. Calcd for $\mathrm{C}_{11} \mathrm{H}_{9} \mathrm{~N}_{3} \mathrm{O}: \mathrm{C}, 66.32 ; \mathrm{H}, 4.55 ; \mathrm{N}, 21.09$. Found: $\mathrm{C}$, $66.45 ; \mathrm{H}, 4.34$; N, 21.09. 


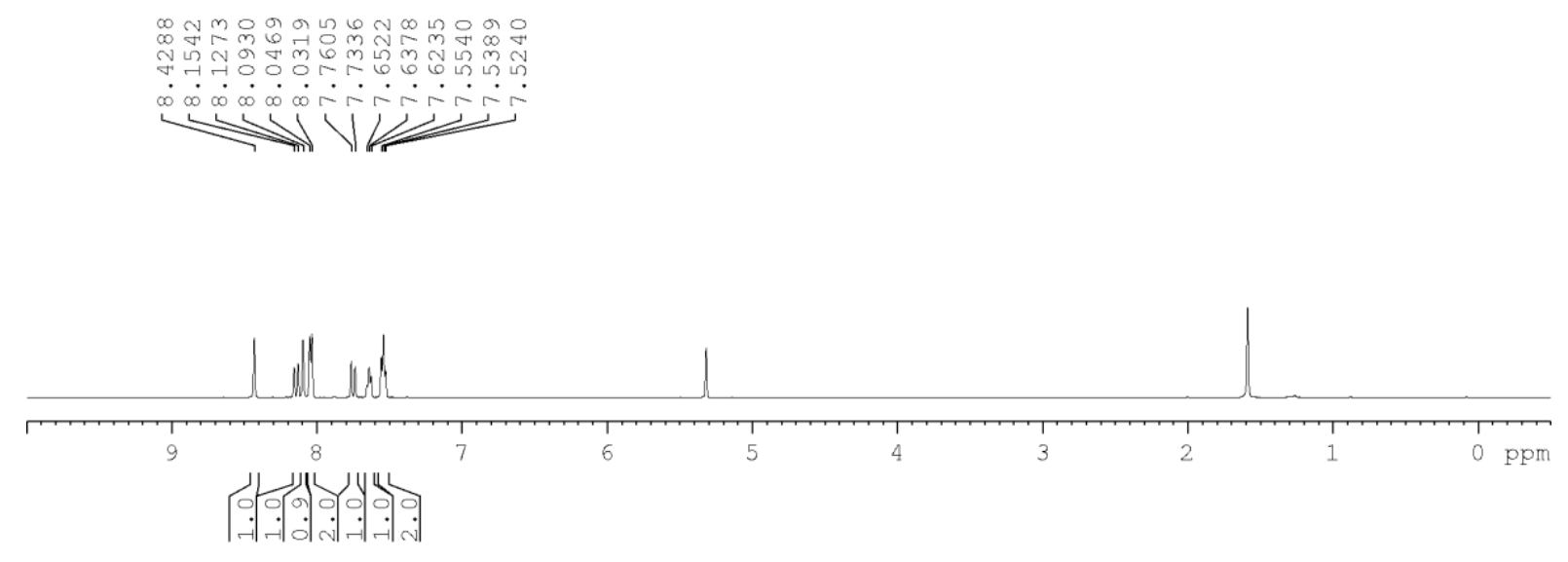

Figure S23: ${ }^{1} \mathrm{H}$ NMR spectrum of $\mathbf{1 h}$

13C $\left(\mathrm{CDCl}_{3}, 75 \mathrm{MHz}\right)$ Triazole substrate

$13 C$ Substrate (triazole)
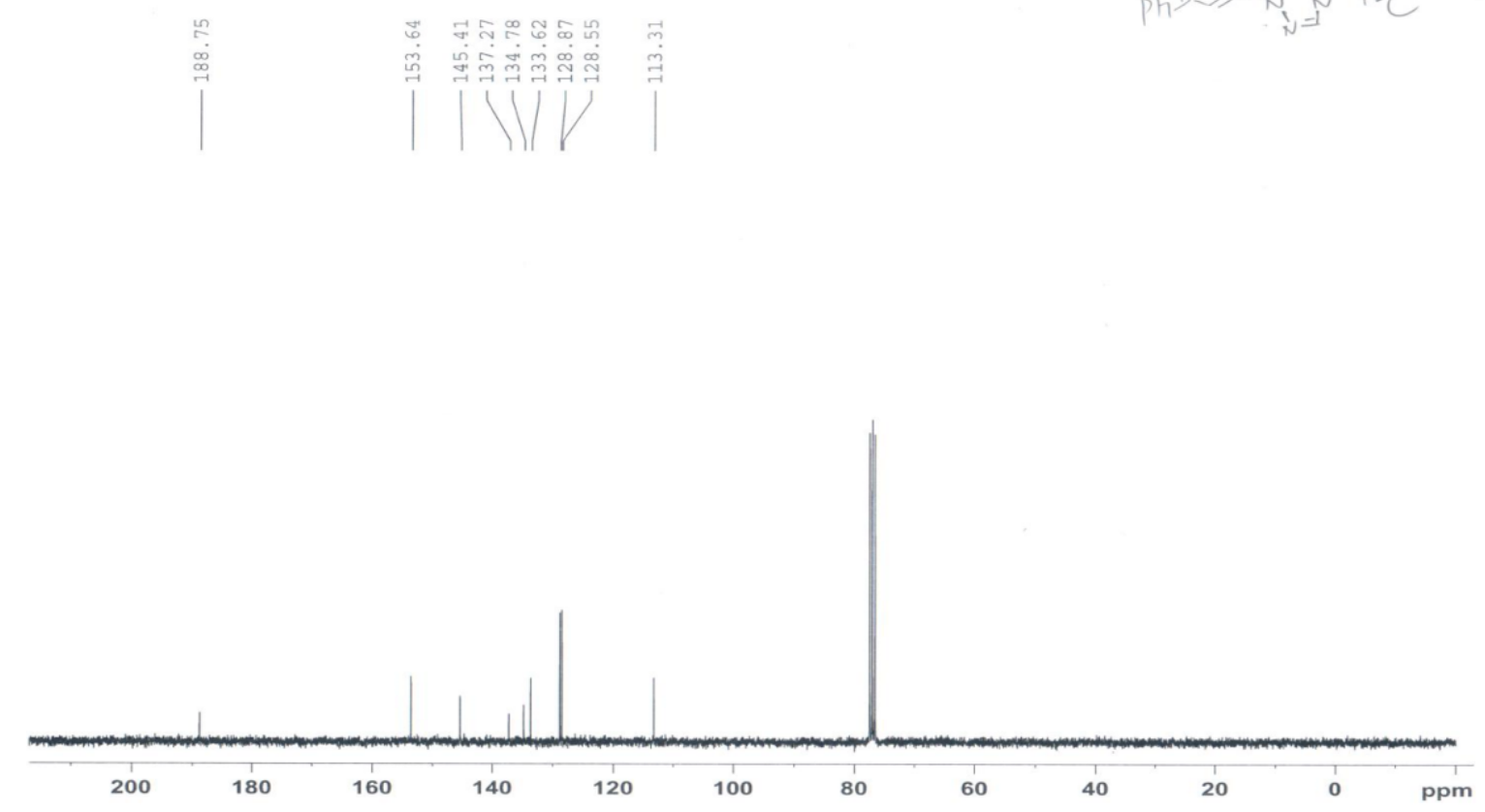

Figure S24: ${ }^{13} \mathrm{C}$ NMR spectrum of $\mathbf{1 h}$ 


\section{General Procedure for asymmetric hydrophosphination}

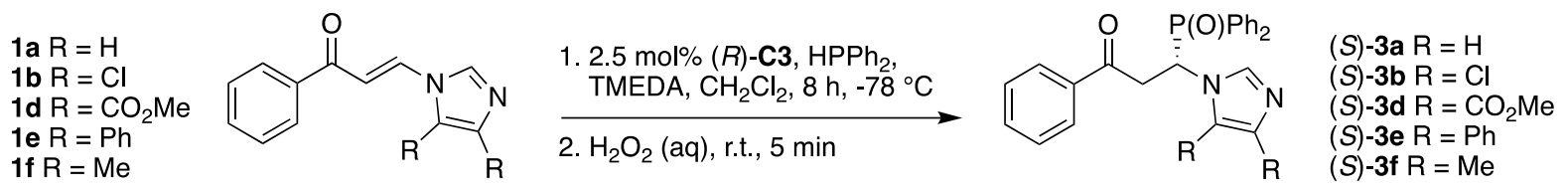

Scheme S4: Synthesis of $(S)-3 \mathbf{a},(S)-3 \mathbf{b},(S)-\mathbf{3 d},(S)-\mathbf{3 e}$ and $(S)-\mathbf{3 f}$.

Diphenylphosphine (10 uL, $0.0575 \mathrm{mmol}, 1$ equiv.), $\mathrm{CH}_{2} \mathrm{Cl}_{2}(575-1150 \mathrm{uL})$ and $(R)-\mathbf{C 3}$ $(0.0009 \mathrm{~g}, 2.5 \mathrm{~mol} \%)$ were added to an oven-dried $10 \mathrm{~mL}$ storage tube and cooled to $-78{ }^{\circ} \mathrm{C}$. Substrates 1 ( $0.0575 \mathrm{mmol}, 1$ equiv.) and tetramethylethylenediamine $(8.6 \mathrm{uL}, 1$ equiv.) were added sequentially into the mixture. The reaction mixture was then stirred at $-78{ }^{\circ} \mathrm{C}$ for the stipulated time. After which, $30 \% \mathrm{w} / \mathrm{w}$ aqueous hydrogen peroxide (1 drop) was added to the mixture and after 5 minutes, the reaction was allowed to warm to room temperature. Volatiles were then removed under reduced pressure and the crude mixture was purified via silica gel chromatography (90 EtOAc:10 MeOH) to afford the pure enantioenriched 3a, 3b, 3d, 3e and 3f.

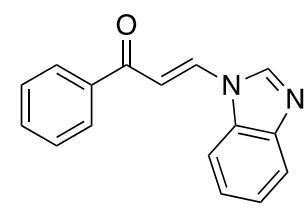

19

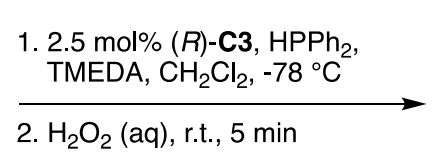

2. $\mathrm{H}_{2} \mathrm{O}_{2}(\mathrm{aq})$, r.t., $5 \mathrm{~min}$

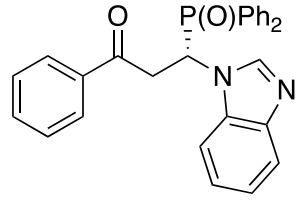

$(S)-\mathbf{3 g}$

Scheme S5: Synthesis of $(S)-3 g$.

Diphenylphosphine (10 uL, $0.0575 \mathrm{mmol}, 1$ equiv.), $\mathrm{CH}_{2} \mathrm{Cl}_{2}(1150 \mathrm{uL})$ and $(R)-\mathbf{C 3}(0.0009 \mathrm{~g}$, $2.5 \mathrm{~mol} \%$ ) were added to an oven-dried $10 \mathrm{~mL}$ storage tube and cooled to $-78{ }^{\circ} \mathrm{C}$. Substrate 1 g $(0.0575 \mathrm{mmol}, 1$ equiv.) and tetramethylethylenediamine ( $8.6 \mathrm{uL}, 1$ equiv.) were added sequentially into the mixture. The reaction mixture was then stirred at $-78{ }^{\circ} \mathrm{C}$ for the stipulated time. After which, $30 \%$ w/w aqueous hydrogen peroxide (1 drop) was added to the mixture and after 5 minutes, the reaction was allowed to warm to room temperature. Volatiles were then removed under reduced pressure and the crude mixture was purified via silica gel chromatography (90 EtOAc:10 $\mathrm{MeOH}$ ) to afford the pure enantioenriched $\mathbf{3 g}$. 


\section{Characterization of enantioenriched products $3 a, b, d, e, f$ and $g$}<smiles>O=C(CC(c1ccccc1)n1ccnc1)c1ccccc1</smiles>

Figure S25. Structure of $(S)-\mathbf{3 a}$

(S)-3a. White solid. Yield: $0.0212 \mathrm{~g}, 0.0529 \mathrm{mmol}, 92 \%$. $[\alpha]_{\mathrm{D}}\left(21{ }^{\circ} \mathrm{C}\right)=-57.2^{\circ} ;{ }^{1} \mathrm{H}$ NMR $\left(\mathrm{CD}_{2} \mathrm{Cl}_{2}, 500 \mathrm{MHz}\right): \delta 3.42\left(\mathrm{ddd}, 1 \mathrm{H}, \mathrm{J}_{\mathrm{HH}}=18.3 \mathrm{~Hz}, 2.4 \mathrm{~Hz}, \mathrm{~J}_{\mathrm{HP}}=7.2 \mathrm{~Hz}\right), 4.06\left(\mathrm{ddd}, 1 \mathrm{H}, \mathrm{J}_{\mathrm{HH}}\right.$ $\left.=18.3 \mathrm{~Hz}, 10.3 \mathrm{~Hz}, \mathrm{~J}_{\mathrm{HP}}=4.1 \mathrm{~Hz}\right), 5.80\left(\mathrm{ddd}, 1 \mathrm{H}, \mathrm{J}_{\mathrm{HH}}=10.4 \mathrm{~Hz}, 2.5 \mathrm{~Hz}, \mathrm{~J}_{\mathrm{HP}}=3.5 \mathrm{~Hz}\right), 6.85(\mathrm{~s}$, $1 \mathrm{H}), 7.26(\mathrm{~s}, 1 \mathrm{H}), 7.42-7.48(\mathrm{~m}, 4 \mathrm{H}), 7.53-7.66(\mathrm{~m}, 8 \mathrm{H}), 7.88-7.90(\mathrm{~m}, 2 \mathrm{H}), 7.96-8.00(\mathrm{~m}, 2 \mathrm{H})$; ${ }^{13} \mathrm{C}\left\{{ }^{1} \mathrm{H}\right\}$ NMR $\left(\mathrm{CD}_{2} \mathrm{Cl}_{2}, 125 \mathrm{MHz}\right): \delta 118.9,128.1,128.7,128.7,128.7,128.9(\mathrm{~d}, \mathrm{~J}=9.7 \mathrm{~Hz})$, 129.0, 129.2, 129.3, 129.7 (d, J = 13.9 Hz), 130.6 (d, J = 9.2 Hz), 131.1 (d, J = 9.0 Hz), 132.5 $(\mathrm{d}, \mathrm{J}=2.8 \mathrm{~Hz}), 132.8(\mathrm{~d}, \mathrm{~J}=2.6 \mathrm{~Hz}), 133.9,135.8,137.8,194.8(\mathrm{~d}, \mathrm{~J}=11.2 \mathrm{~Hz}) ;{ }^{31} \mathrm{P}\left\{{ }^{1} \mathrm{H}\right\} \mathrm{NMR}$ $\left(\mathrm{CH}_{2} \mathrm{Cl}_{2}, 121 \mathrm{MHz}\right): \delta 30.63$; HRMS (+ESI) m/z: $(\mathrm{M}+\mathrm{H})+$ calcd for $\mathrm{C}_{24} \mathrm{H}_{22} \mathrm{~N}_{2} \mathrm{O}_{2} \mathrm{P}, 401.1419$; found, 401.1420 .

1H(CD2C12, AV500) substrate (H2) $\mathrm{P}=\mathrm{O}$

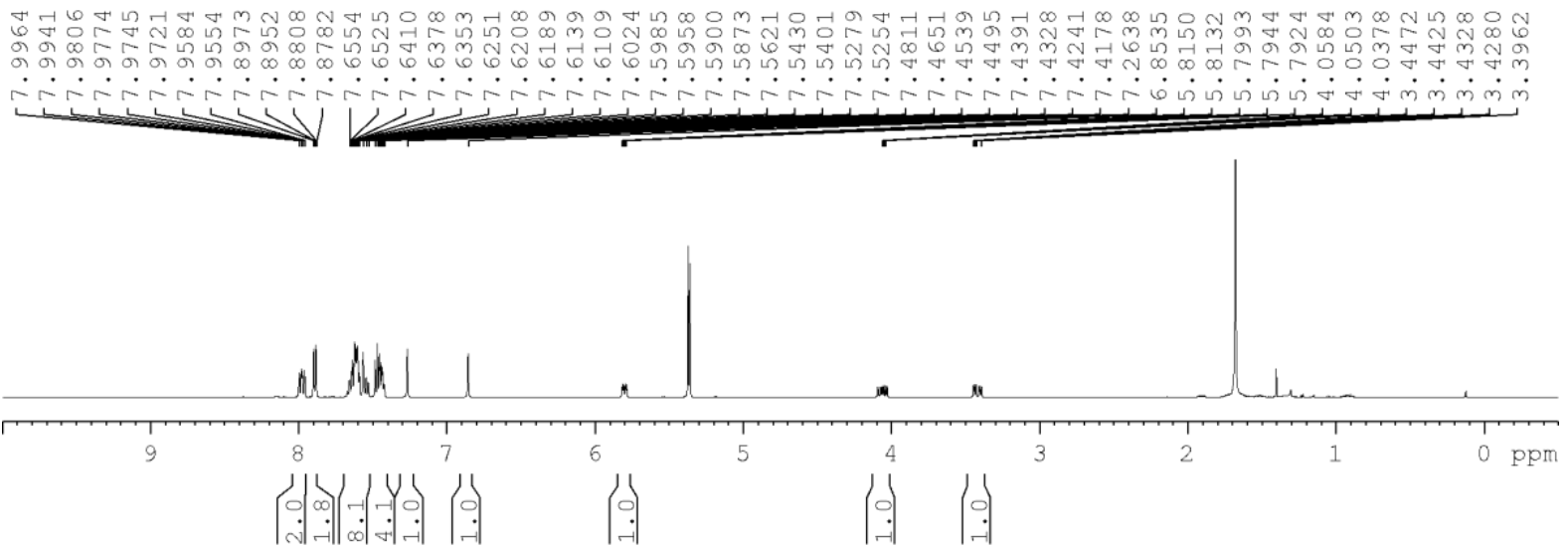

Figure S26: ${ }^{1} \mathrm{H}$ NMR spectrum of $\mathbf{3 a}$ 


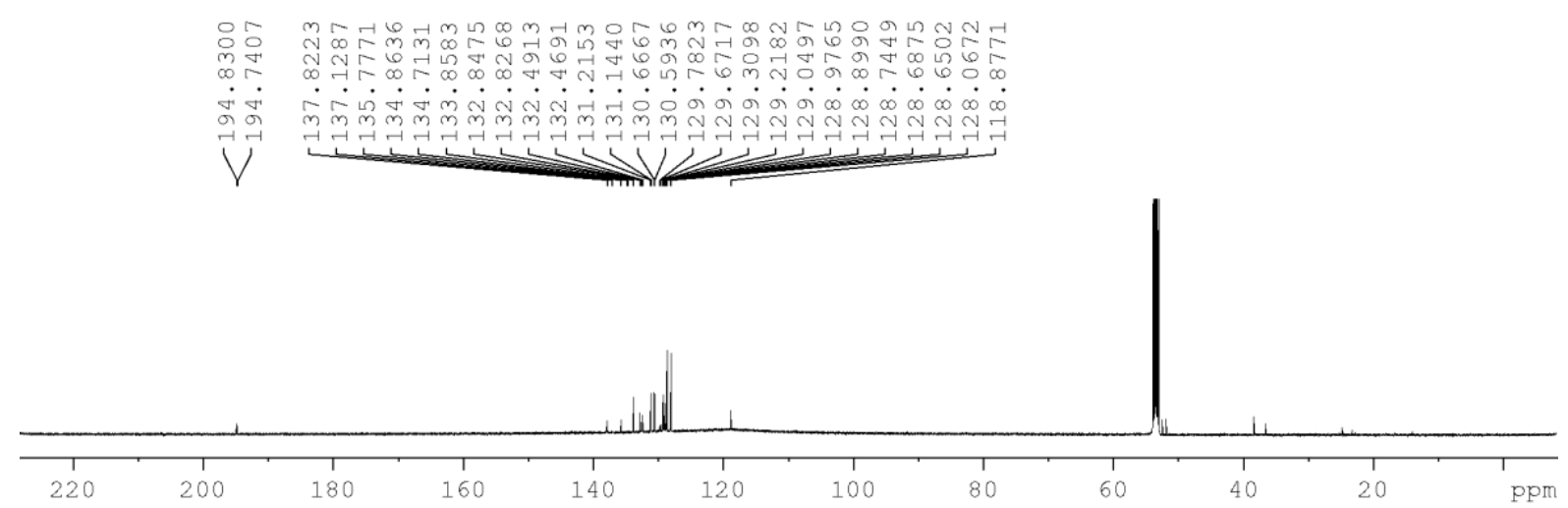

Figure S27: ${ }^{13} \mathrm{C}$ NMR spectrum of 3a

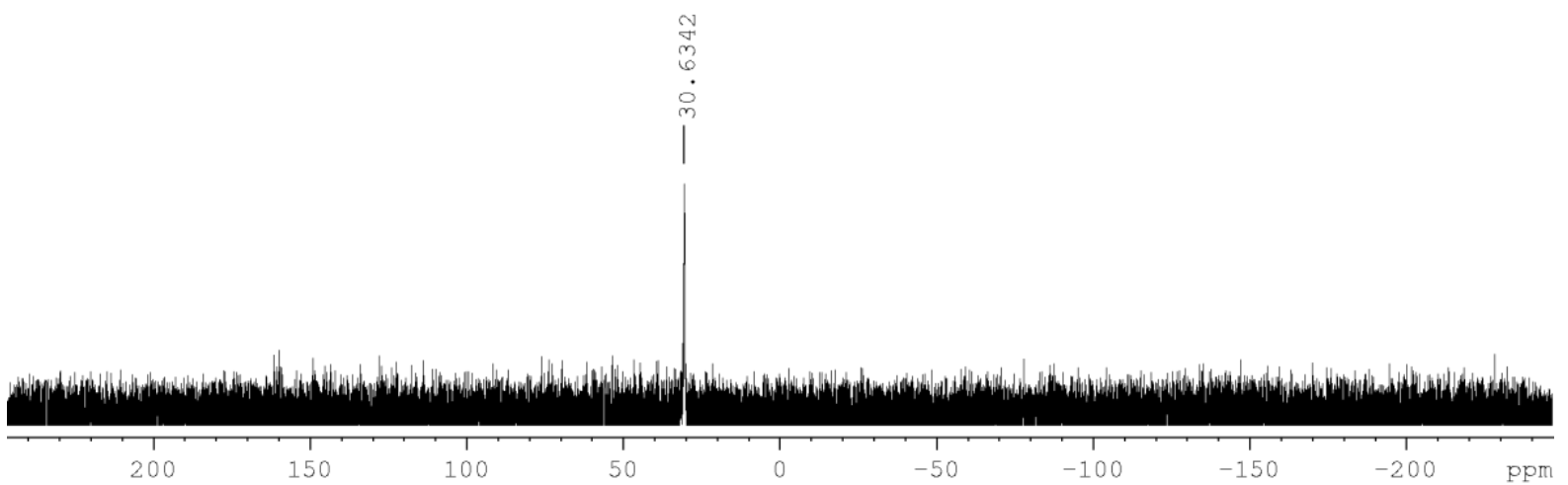

Figure S28: ${ }^{31} \mathrm{P}\left\{{ }^{1} \mathrm{H}\right\}$ NMR spectrum of 3a 
IC column, $1 \mathrm{~mL} / \mathrm{min}, 60$ Hexane: $40 \mathrm{i} \mathrm{PrOH}, 30^{\circ} \mathrm{C}$

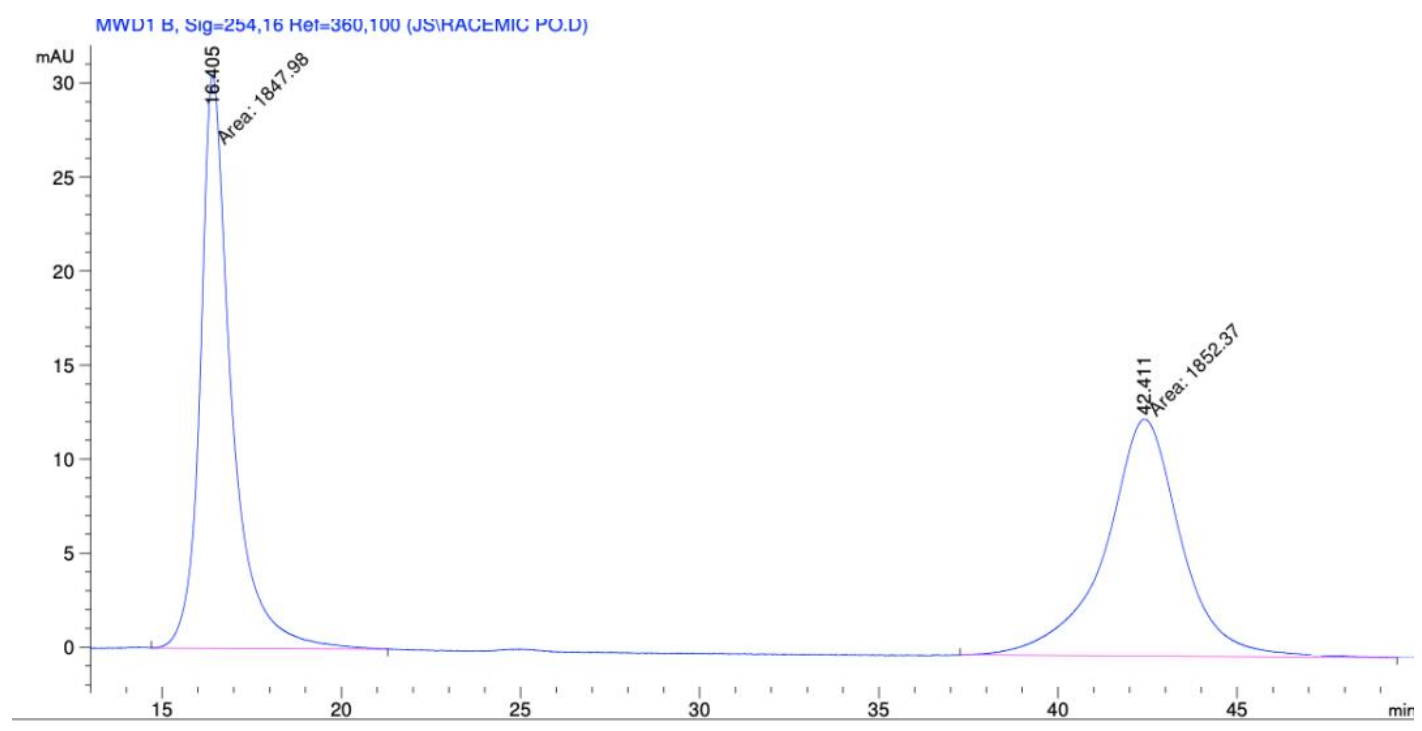

Signal 1: MWD1 B, Sig=254,16 Ref $=360,100$

\begin{tabular}{|c|c|c|c|c|c|c|}
\hline $\begin{array}{c}\text { Peak } \\
\quad \#\end{array}$ & $\begin{array}{l}\text { RetTime } \\
{[\text { min] }}\end{array}$ & Type & $\begin{array}{l}\text { Width } \\
{[\mathrm{min}]}\end{array}$ & $\begin{array}{c}\text { Area } \\
{\left[\mathrm{mAU}{ }^{*} \mathrm{~s}\right]}\end{array}$ & $\begin{array}{l}\text { Height } \\
\text { [MAU] }\end{array}$ & $\begin{array}{c}\text { Area } \\
\frac{\circ}{0}\end{array}$ \\
\hline 1 & 16.405 & MM T & 1.0098 & 1847.97974 & 30.50218 & 49.9407 \\
\hline 2 & 42.411 & MM T & 2.4551 & 1852.36938 & 12.57513 & 50.0593 \\
\hline Total & Is : & & & 3700.34912 & 43.07731 & \\
\hline
\end{tabular}

Figure S29: Chiral HPLC spectrum of racemic 3a 


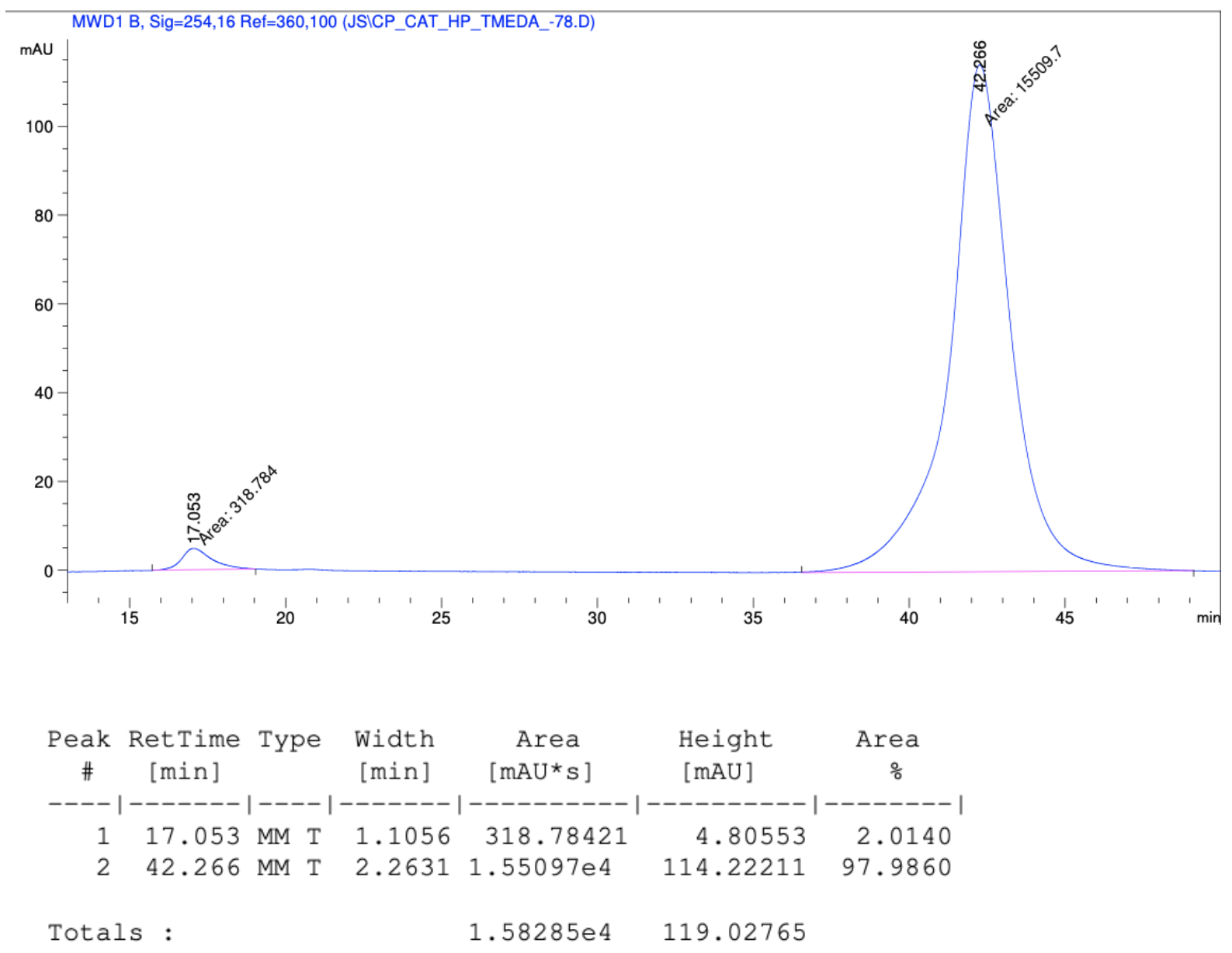

Figure S30: Chiral HPLC spectrum of enantioenriched 3a 
<smiles>O=C(CC(n1ccnc1)n1cnc(Cl)c1Cl)c1ccccc1</smiles>

Figure S31. Structure of $(S)-3 \mathbf{b}$

(S)-3b. White solid. Yield: $0.0211 \mathrm{~g}, 0.0449 \mathrm{mmol}, 78 \%$. $[\alpha]_{\mathrm{D}}\left(21{ }^{\circ} \mathrm{C}\right)=-169.2^{\circ} ;{ }^{1} \mathrm{H} \mathrm{NMR}$ $\left(\mathrm{CD}_{2} \mathrm{Cl}_{2}, 300 \mathrm{MHz}\right): \delta 3.50\left(\mathrm{ddd}, 1 \mathrm{H}, \mathrm{J}_{\mathrm{HH}}=18.5 \mathrm{~Hz}, 2.8 \mathrm{~Hz}, \mathrm{~J}_{\mathrm{HP}}=6.7 \mathrm{~Hz}\right), 4.06\left(\mathrm{ddd}, 1 \mathrm{H}, \mathrm{J}_{\mathrm{HH}}\right.$ $\left.=18.4 \mathrm{~Hz}, 10.3 \mathrm{~Hz}, \mathrm{~J}_{\mathrm{HP}}=5.0 \mathrm{~Hz}\right), 5.75\left(\mathrm{ddd}, 1 \mathrm{H}, \mathrm{J}_{\mathrm{HH}}=10.2 \mathrm{~Hz}, 2.7 \mathrm{~Hz}, \mathrm{~J}_{\mathrm{HP}}=3.0 \mathrm{~Hz}\right), 7.41-$ $7.65(\mathrm{~m}, 11 \mathrm{H}), 7.83-8.01(\mathrm{~m}, 5 \mathrm{H}) ;{ }^{13} \mathrm{C}\left\{{ }^{1} \mathrm{H}\right\} \mathrm{NMR}\left(\mathrm{CDCl}_{3}, 100 \mathrm{MHz}\right): \delta 38.6(\mathrm{~d}, \mathrm{~J}=4.9 \mathrm{~Hz})$, 50.6, 51.3, $114.6(\mathrm{~d}, \mathrm{~J}=2.0 \mathrm{~Hz}), 125.9,127.7(\mathrm{~d}, \mathrm{~J}=3.3 \mathrm{~Hz}), 128.3,128.7(\mathrm{~d}, \mathrm{~J}=8.3 \mathrm{~Hz})$, 129.0, $129.0(\mathrm{~d}, \mathrm{~J}=12.2 \mathrm{~Hz}), 129.6(\mathrm{~d}, \mathrm{~J}=11.7 \mathrm{~Hz}), 130.8(\mathrm{~d}, \mathrm{~J}=9.6 \mathrm{~Hz}), 131.6$ (d, J = 8.8 $\mathrm{Hz}), 133.3(\mathrm{dd}, \mathrm{J}=2.9 \mathrm{~Hz}, 23.1 \mathrm{~Hz}), 133.9,134.3,135.5,194.1(\mathrm{~d}, \mathrm{~J}=10.5) ;{ }^{31} \mathrm{P}\left\{{ }^{1} \mathrm{H}\right\} \mathrm{NMR}$ $\left(\mathrm{CH}_{2} \mathrm{Cl}_{2}, 121 \mathrm{MHz}\right): \delta$ 31.88; HRMS $(+\mathrm{ESI}) \mathrm{m} / \mathrm{z}$ : $(\mathrm{M}+\mathrm{H})+$ calcd for $\mathrm{C}_{24} \mathrm{H}_{20} \mathrm{~N}_{2} \mathrm{O}_{2} \mathrm{PCl}_{2}$, 469.0639; found, 469.0639 .

$1 \mathrm{H}(\mathrm{CD} 2 \mathrm{Cl2}, 300 \mathrm{MHz}) \mathrm{Cl} 2 \mathrm{P}=\mathrm{O}$
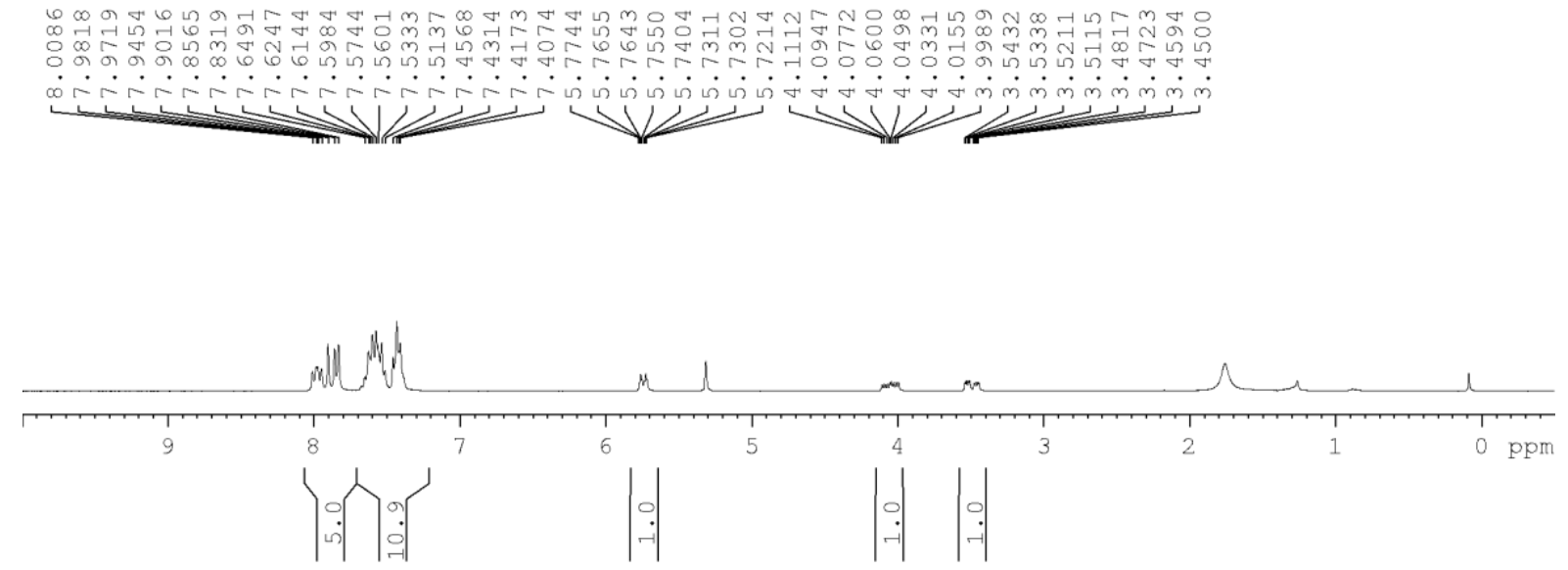

Figure S32: ${ }^{1} \mathrm{H}$ NMR spectrum of $\mathbf{3 b}$ 

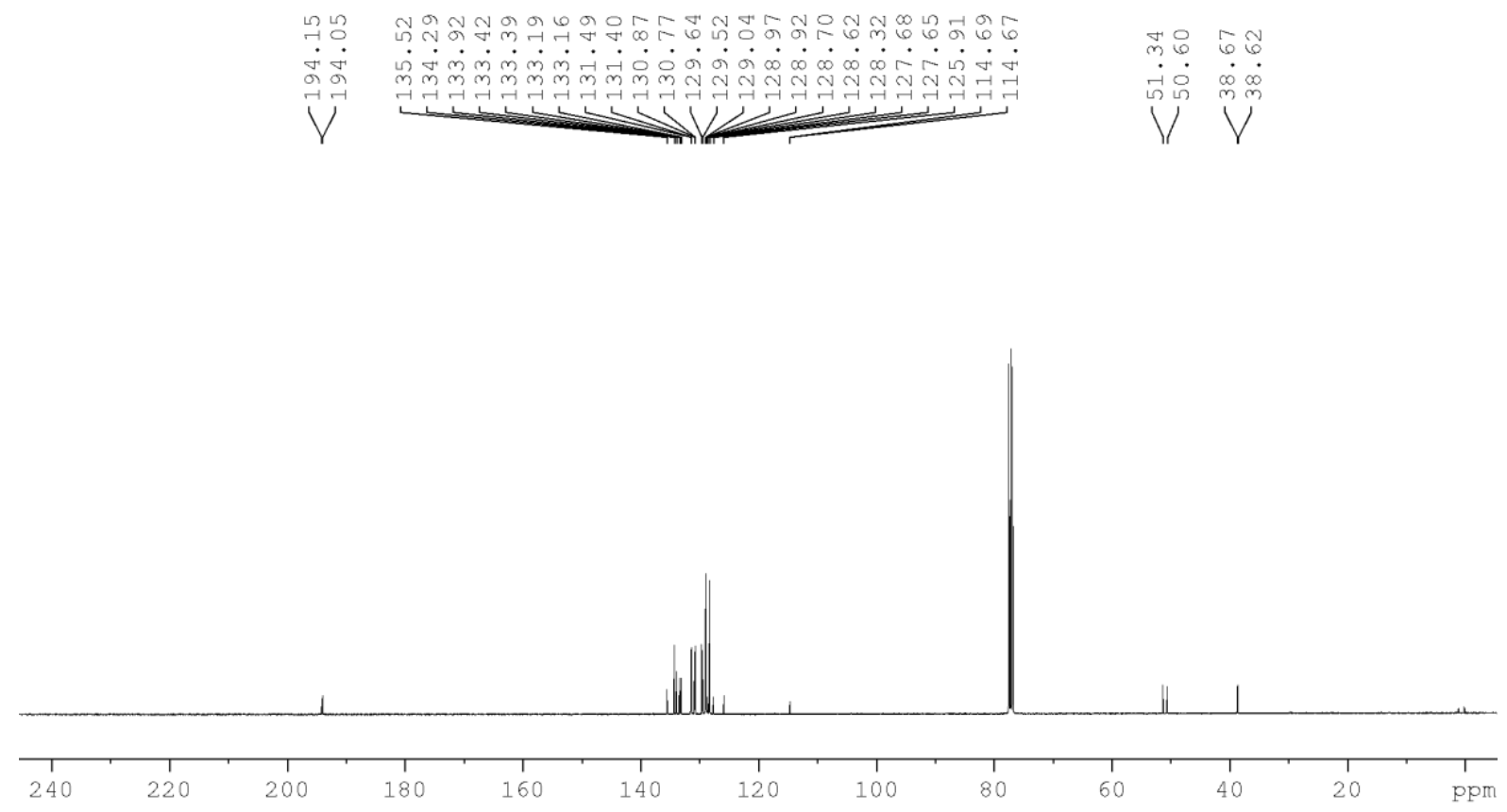

Figure S33: ${ }^{13} \mathrm{C}$ NMR spectrum of $\mathbf{3 b}$

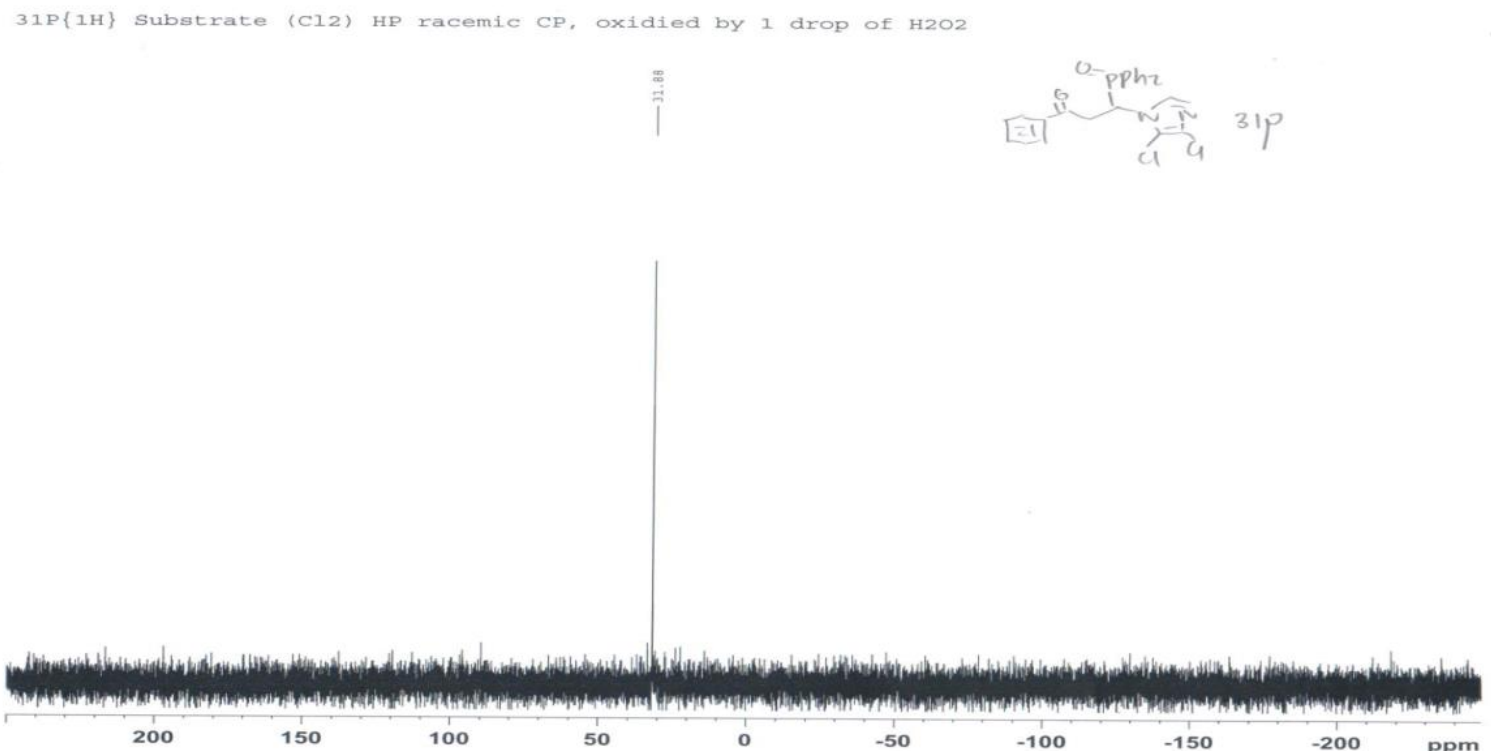

Figure S34: ${ }^{31} \mathrm{P}\left\{{ }^{1} \mathrm{H}\right\}$ NMR spectrum of $\mathbf{3 b}$ 
ID column, $1 \mathrm{~mL} / \mathrm{min}, 60$ Hexane: $40 \mathrm{i} \mathrm{PrOH}, 30^{\circ} \mathrm{C}$
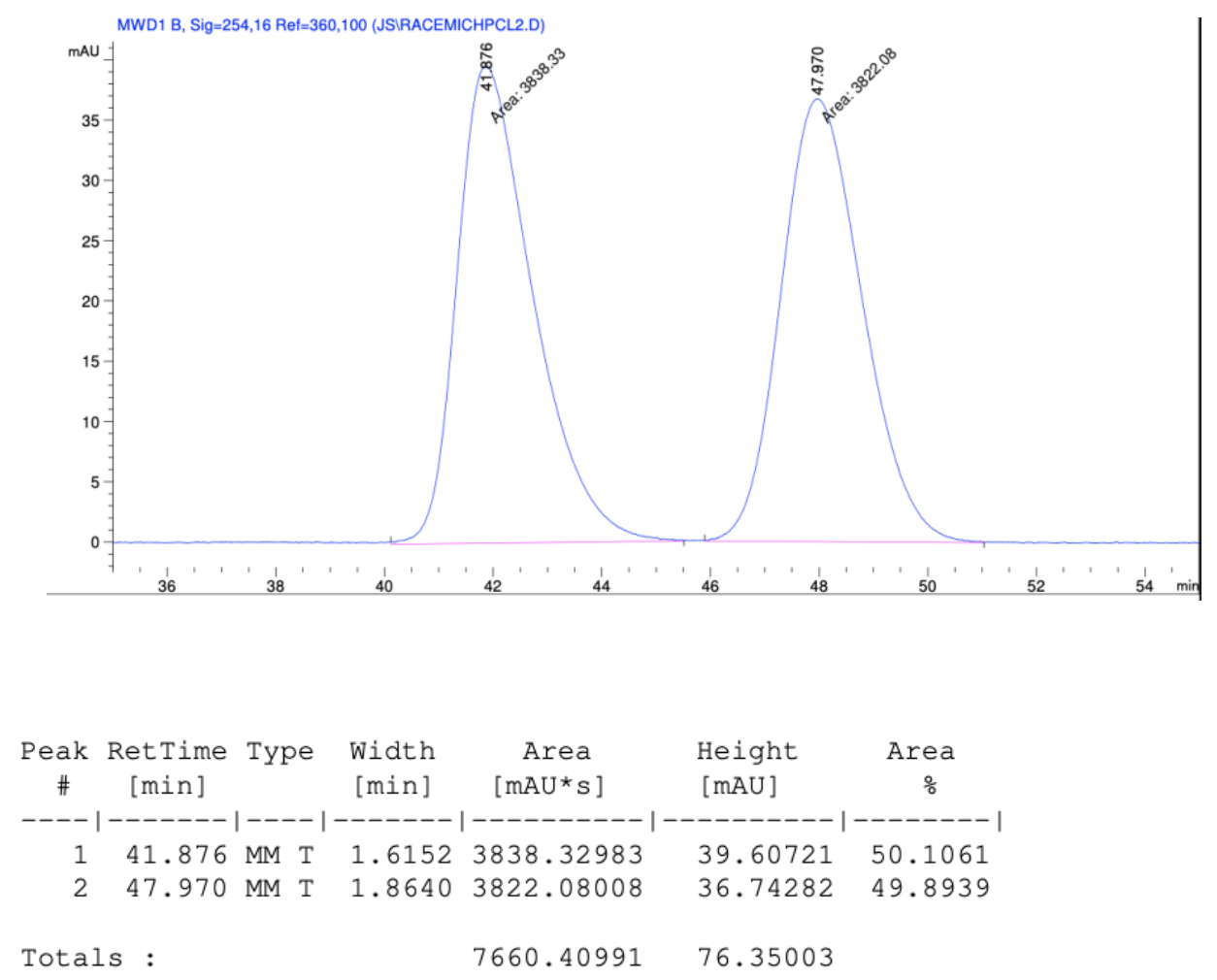

Figure S35: Chiral HPLC spectrum of racemic 3b
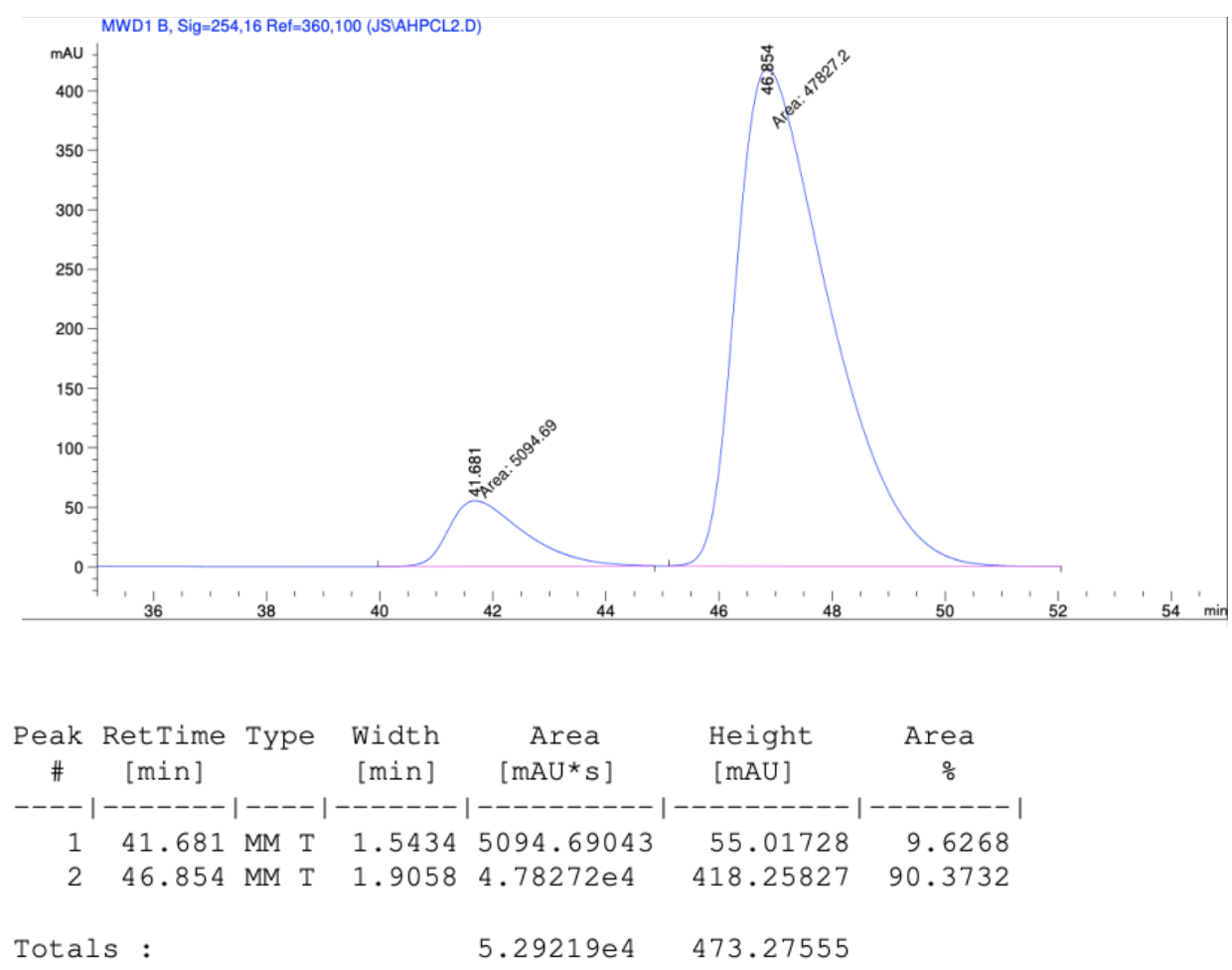

Figure S36: Chiral HPLC spectrum of enantioenriched 3b 
<smiles>COC(=O)c1ncn(C(CC(=O)c2ccccc2)c2ccccc2)c1C(C)=O</smiles>

Figure S37. Structure of $(S)$-3d

(S)-3d. White solid. Yield: $0.0226 \mathrm{~g}, 0.0437 \mathrm{mmol}, 76 \%$. $[\alpha]_{\mathrm{D}}\left(21{ }^{\circ} \mathrm{C}\right)=-131.8^{\circ} ;{ }^{1} \mathrm{H}$ NMR $\left(\mathrm{CD}_{2} \mathrm{Cl}_{2}, 300 \mathrm{MHz}\right): \delta 3.46\left(\mathrm{ddd}, 1 \mathrm{H}, \mathrm{J}_{\mathrm{HH}}=18.5 \mathrm{~Hz}, 2.6 \mathrm{~Hz}, \mathrm{~J}_{\mathrm{HP}}=5.9 \mathrm{~Hz}\right), 3.78(\mathrm{~s}, 3 \mathrm{H}, \mathrm{Me})$, $3.91(\mathrm{~s}, 3 \mathrm{H}, \mathrm{Me}), 4.08\left(\mathrm{ddd}, 1 \mathrm{H}, \mathrm{J}_{\mathrm{HH}}=18.4 \mathrm{~Hz}, 11.0 \mathrm{~Hz}, \mathrm{~J}_{\mathrm{HP}}=5.0 \mathrm{~Hz}\right), 6.67\left(\mathrm{ddd}, 1 \mathrm{H}, \mathrm{J}_{\mathrm{HH}}=\right.$ $\left.10.9 \mathrm{~Hz}, 2.6 \mathrm{~Hz}, \mathrm{~J}_{\mathrm{HP}}=3.9 \mathrm{~Hz}\right), 7.36-7.76(\mathrm{~m}, 13 \mathrm{H}), 8.00-8.06(\mathrm{~m}, 2 \mathrm{H}), 8.15(\mathrm{~s}, 1 \mathrm{H}) ;{ }^{13} \mathrm{C}\left\{{ }^{1} \mathrm{H}\right\}$ NMR $\left(\mathrm{CDCl}_{3}, 100 \mathrm{MHz}\right): \delta 39.3(\mathrm{~d}, \mathrm{~J}=4.2 \mathrm{~Hz}), 50.4,51.1,52.3,52.6,52.9,126.4,127.7$, 128.3, 128.4, 128.8, 128.9, 129.5 (d, J =11.8 Hz), 131.2 (dd, J = 26.4 Hz, 9.4 Hz), 133.0 (dd, $\mathrm{J}=34.0 \mathrm{~Hz}, 2.6 \mathrm{~Hz}), 134.1,135.6,135.7,138.4,161.5,162.4,194.5(\mathrm{~d}, \mathrm{~J}=11.5 \mathrm{~Hz}) ;{ }^{31} \mathrm{P}\left\{{ }^{1} \mathrm{H}\right\}$ NMR $\left(\mathrm{CH}_{2} \mathrm{Cl}_{2}, 121 \mathrm{MHz}\right): \delta 32.53$; HRMS (+ESI) m/z: $(\mathrm{M}+\mathrm{H})+$ calcd for $\mathrm{C}_{28} \mathrm{H}_{26} \mathrm{~N}_{2} \mathrm{O} 6 \mathrm{P}$, 517.1529; found, 517.1528.

$1 \mathrm{H}(\mathrm{CD} 2 \mathrm{Cl} 2,300 \mathrm{MHz})(\mathrm{CO} 2 \mathrm{Me}) 2 \mathrm{P}=0$

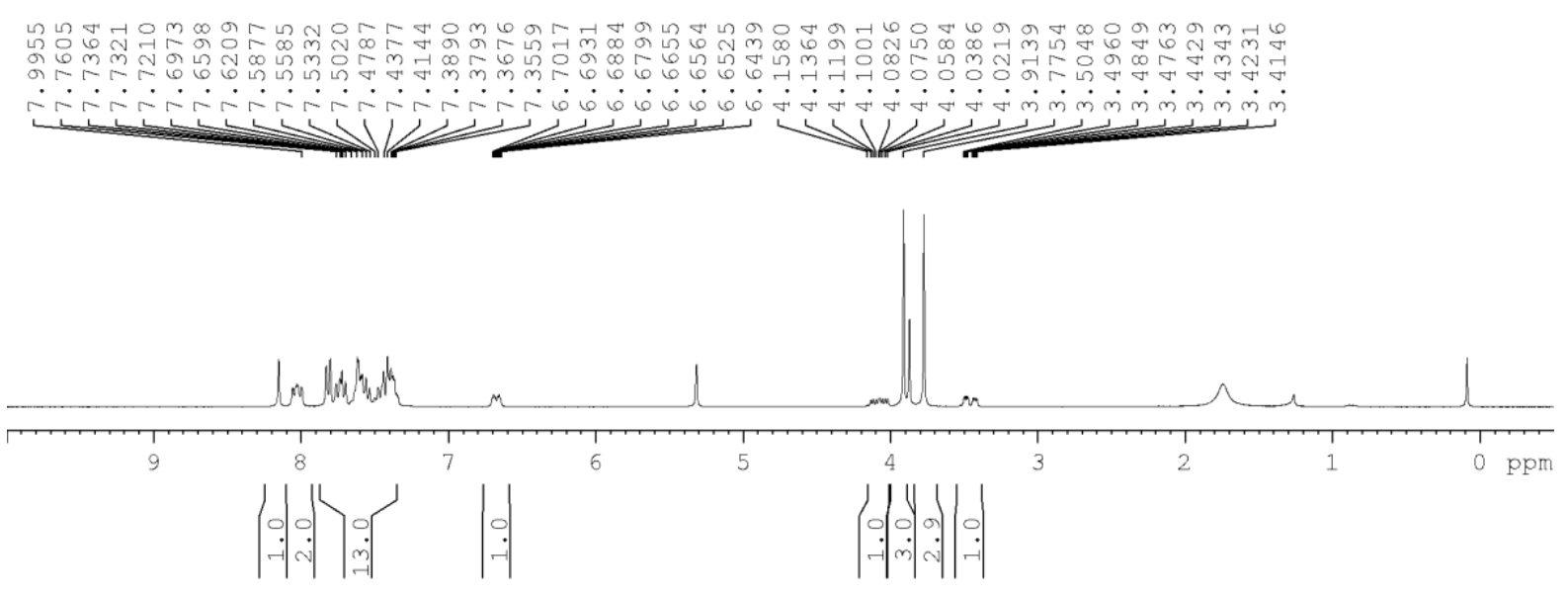

Figure S38: ${ }^{1} \mathrm{H}$ NMR spectrum of $\mathbf{3 d}$ 

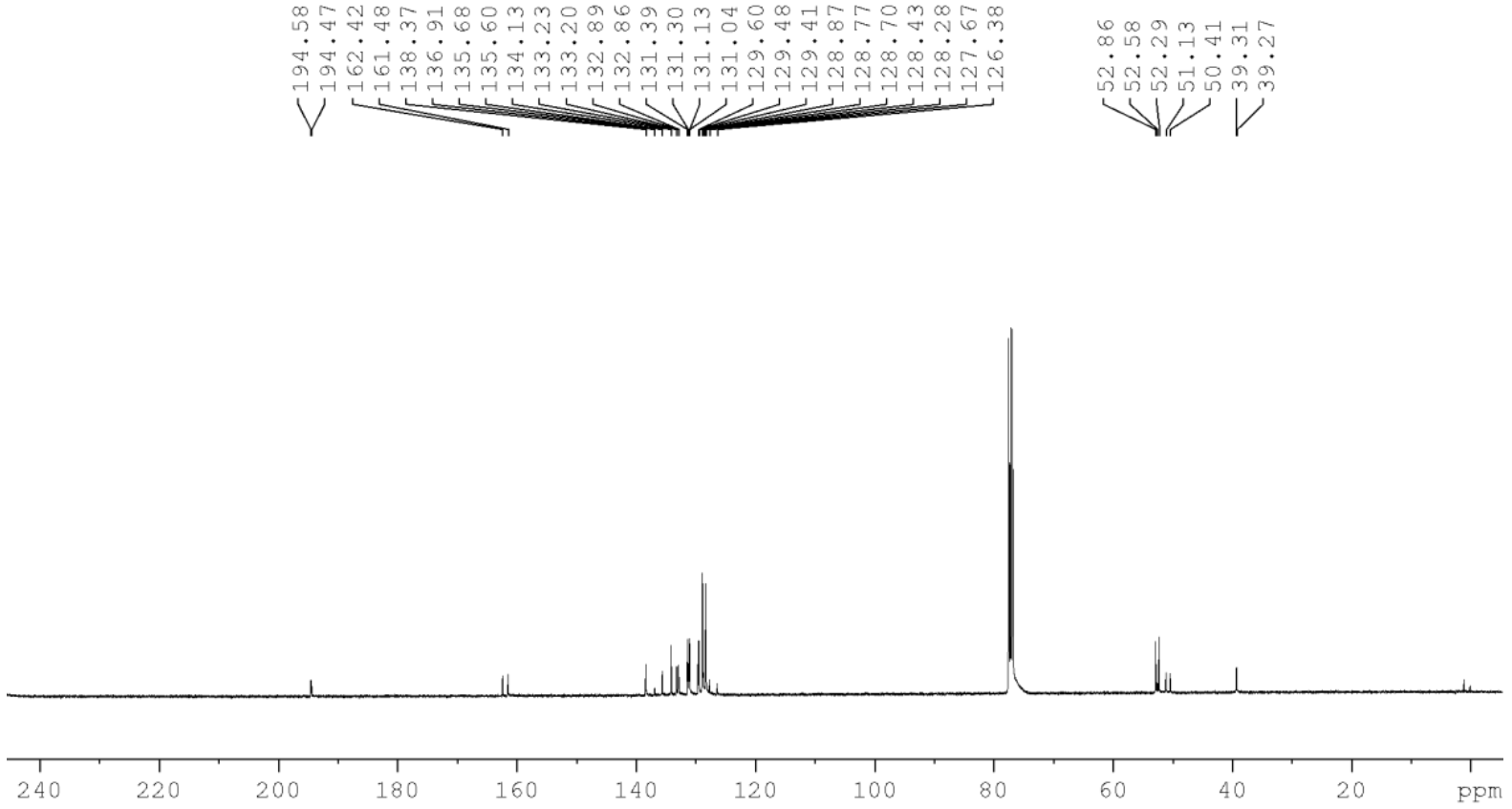

Figure S39: ${ }^{13} \mathrm{C}$ NMR spectrum of $\mathbf{3 d}$

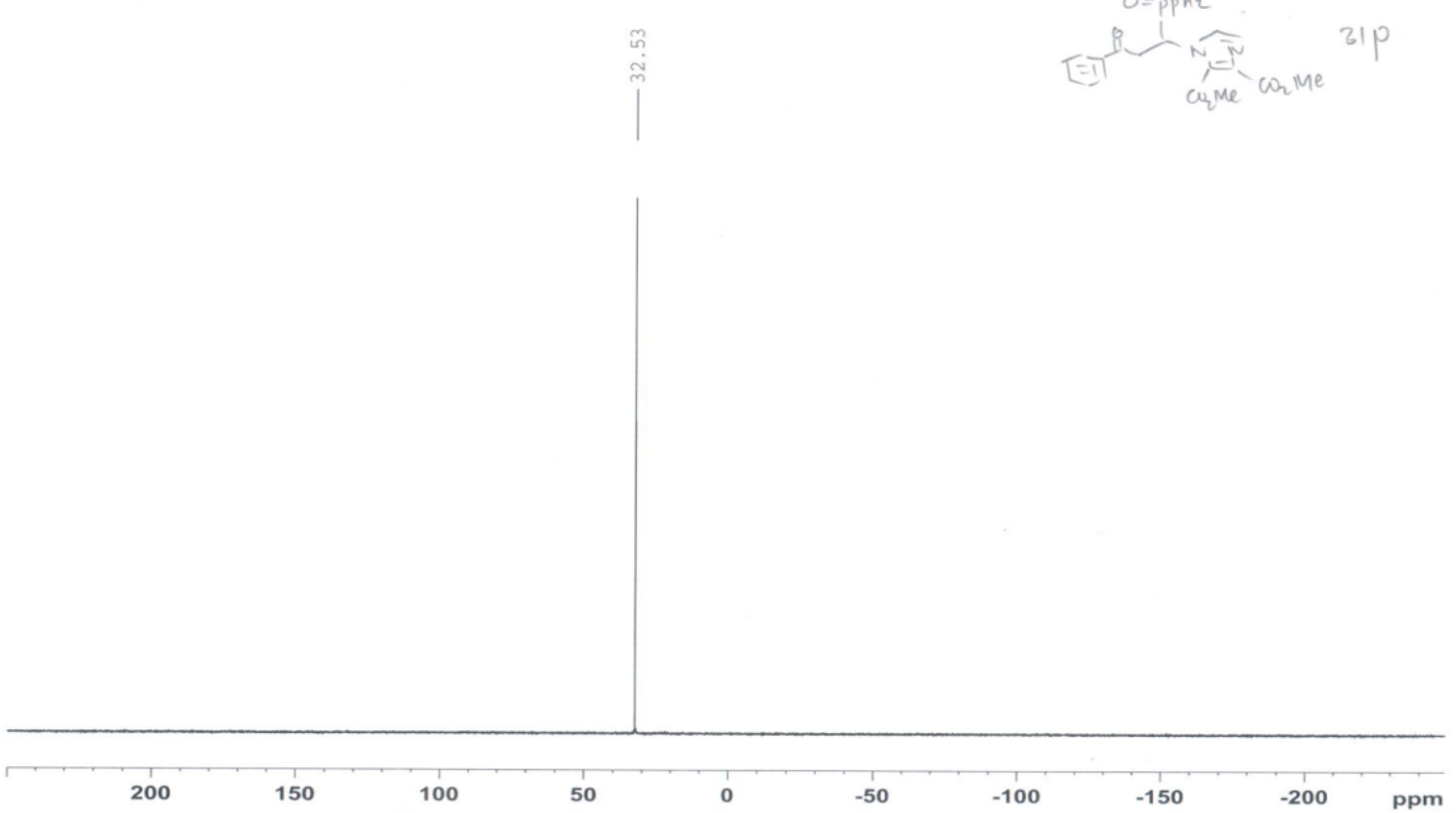

Figure S40: ${ }^{31} \mathrm{P}\left\{{ }^{1} \mathrm{H}\right\}$ NMR spectrum of $\mathbf{3 d}$ 
ID column, $1 \mathrm{~mL} / \mathrm{min}, 60$ Hexane: $40 \mathrm{i} \mathrm{PrOH}, 30^{\circ} \mathrm{C}$

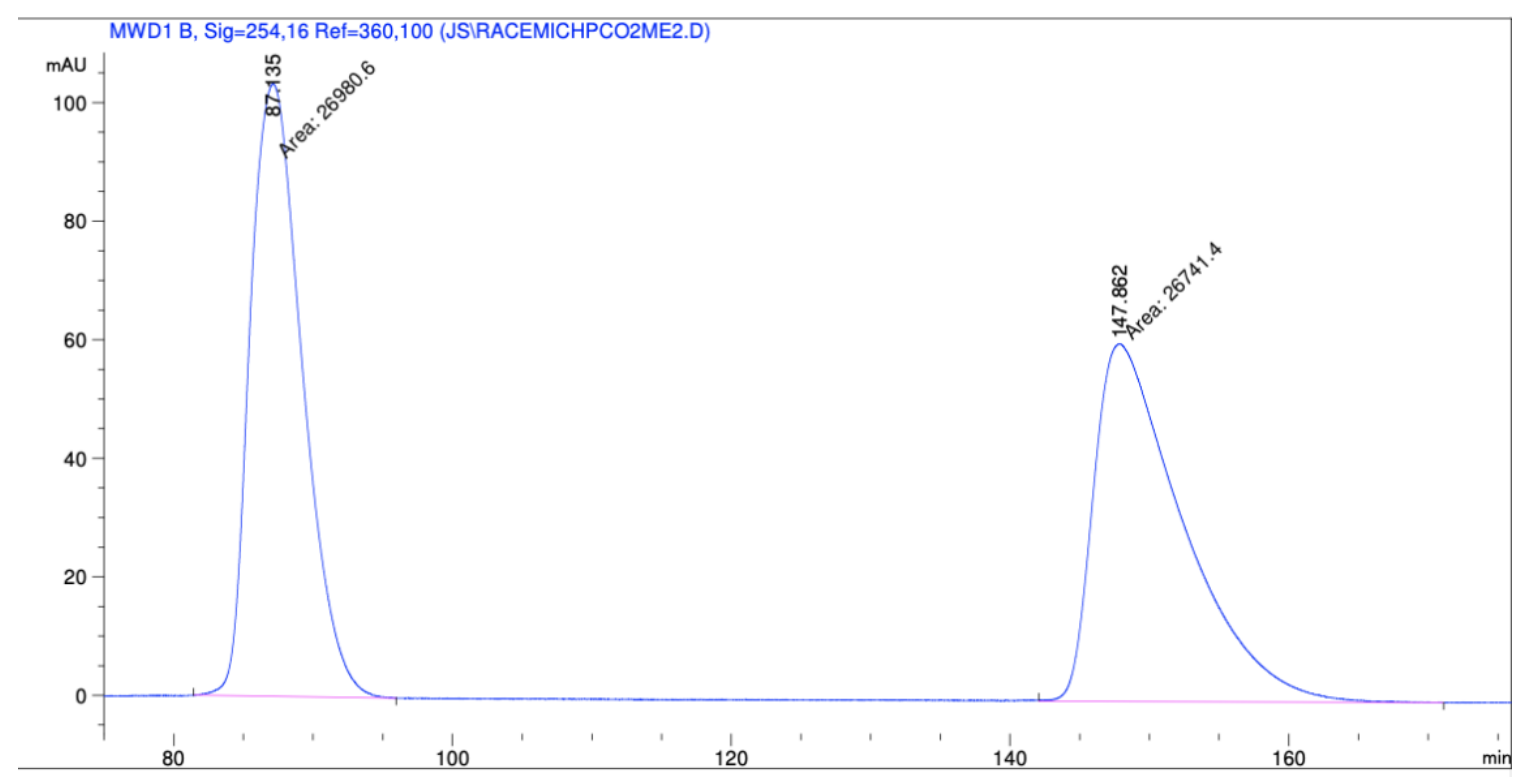

\begin{tabular}{|c|c|c|c|c|c|c|}
\hline $\begin{array}{c}\text { Peak } \\
\#\end{array}$ & $\begin{array}{c}\text { RetTime } \\
\text { [min] }\end{array}$ & Type & $\begin{array}{l}\text { Width } \\
\text { [min] }\end{array}$ & $\begin{array}{c}\text { Area } \\
{\left[\mathrm{mAU}^{\star} \mathrm{s}\right]}\end{array}$ & $\begin{array}{l}\text { Height } \\
\text { [mAU] }\end{array}$ & $\begin{array}{c}\text { Area } \\
\frac{\%}{\delta}\end{array}$ \\
\hline 1 & 87.135 & $\mathrm{MM} \mathrm{T}$ & 4.3515 & $2.69806 \mathrm{e} 4$ & 103.33842 & 50.2226 \\
\hline 2 & 147.862 & $\mathrm{MM} \mathrm{T}$ & 7.3865 & $2.67414 \mathrm{e} 4$ & 60.33841 & 49.7774 \\
\hline Tot & : & & & $5.37220 e^{4}$ & 163.67683 & \\
\hline
\end{tabular}

Figure S41: Chiral HPLC spectrum of racemic 3d 


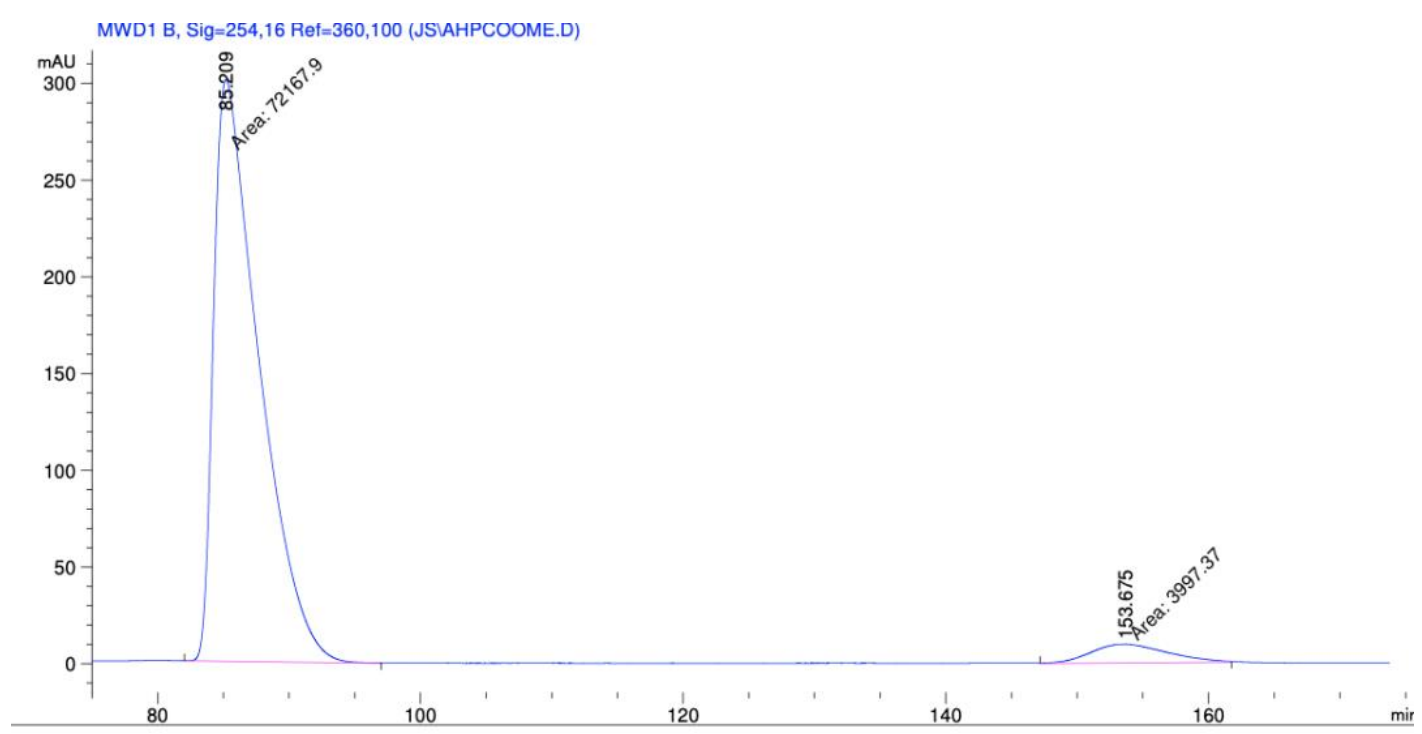

\begin{tabular}{|c|c|c|c|c|c|c|}
\hline $\begin{array}{c}\text { Peak } \\
\#\end{array}$ & $\begin{array}{c}\text { RetTime } \\
\text { [min] }\end{array}$ & Type & $\begin{array}{l}\text { Width } \\
\text { [min] }\end{array}$ & $\begin{array}{c}\text { Area } \\
{\left[\mathrm{mAU}^{*} \mathrm{~s}\right]}\end{array}$ & $\begin{array}{l}\text { Height } \\
{[\mathrm{mAU}]}\end{array}$ & $\begin{array}{c}\text { Area } \\
\%\end{array}$ \\
\hline & & & & & & \\
\hline $\begin{array}{l}1 \\
2\end{array}$ & $\begin{array}{r}85.209 \\
153.675\end{array}$ & $\begin{array}{ll}\text { MM } & 1 \\
\text { MM T } & \text { T }\end{array}$ & $\begin{array}{l}3.9992 \\
6.7913\end{array}$ & $\begin{array}{l}1.21619 e 4 \\
3997 \quad 36621\end{array}$ & 9.80996 & $\begin{array}{r}94.1511 \\
5.2483\end{array}$ \\
\hline ota. & : & & & $7.61653 \mathrm{e} 4$ & 310.57125 & \\
\hline
\end{tabular}

Figure S42: Chiral HPLC spectrum of enantioenriched 3d 
<smiles>O=C(C[C@H](n1cnc(-c2ccccc2)c1-c1ccccc1)P(=O)(O)c1ccccc1)c1ccccc1</smiles>

Figure S43. Structure of $(S)$-3e

(S)-3e. White solid. Yield: $0.0283 \mathrm{~g}, 0.0512 \mathrm{mmol}, 89 \%$. $[\alpha]_{\mathrm{D}}\left(21{ }^{\circ} \mathrm{C}\right)=-83.6^{\circ} ;{ }^{1} \mathrm{H} \mathrm{NMR}$ $\left(\mathrm{CD}_{2} \mathrm{Cl}_{2}, 300 \mathrm{MHz}\right): \delta 3.50\left(\mathrm{ddd}, 1 \mathrm{H}, \mathrm{J}_{\mathrm{HH}}=18.1 \mathrm{~Hz}, 3.6 \mathrm{~Hz}, \mathrm{~J}_{\mathrm{HP}}=8.1 \mathrm{~Hz}\right), 4.11\left(\mathrm{ddd}, 1 \mathrm{H}, \mathrm{J}_{\mathrm{HH}}\right.$ $\left.=18.0 \mathrm{~Hz}, 9.4 \mathrm{~Hz}, \mathrm{~J}_{\mathrm{HP}}=6.7 \mathrm{~Hz}\right), 5.46\left(\mathrm{ddd}, 1 \mathrm{H}, \mathrm{J}_{\mathrm{HH}}=9.2 \mathrm{~Hz}, 3.5 \mathrm{~Hz}, \mathrm{~J}_{\mathrm{HP}}=2.7 \mathrm{~Hz}\right), 7.10-7.85$ $(\mathrm{m}, 25 \mathrm{H}), 8.23(\mathrm{~s}, 1 \mathrm{H}) ;{ }^{13} \mathrm{C}\left\{{ }^{1} \mathrm{H}\right\} \mathrm{NMR}\left(\mathrm{CDCl}_{3}, 100 \mathrm{MHz}\right): \delta 39.2(\mathrm{~d}, \mathrm{~J}=5.0 \mathrm{~Hz}), 50.2,50.9$, 126.5, 126.8, 127.3, 127.9, 128.0, $128.2(\mathrm{~d}, \mathrm{~J}=23.5 \mathrm{~Hz}), 128.8(\mathrm{~d}, \mathrm{~J}=17.8 \mathrm{~Hz}), 128.8,128.9$, 129.0, 129.3 (d, J = 11.6 Hz), 129.4, 129.5, 129.6, 129.7, 131.5, 131.6 (q, J = 8.8 Hz), 132.9 (d, J = 2.8 Hz), 133.1 (d, J = 2.5 Hz), 134.0, 134.4, 135.9, 136.0, 137.8, 194.8 (d, J =10.3 Hz); ${ }^{31} \mathrm{P}\left\{{ }^{1} \mathrm{H}\right\}$ NMR $\left(\mathrm{CH}_{2} \mathrm{Cl}_{2}, 121 \mathrm{MHz}\right): \delta$ 31.44; HRMS (+ESI) $\mathrm{m} / \mathrm{z}:(\mathrm{M}+\mathrm{H})+$ calcd for $\mathrm{C}_{36} \mathrm{H}_{30} \mathrm{~N}_{2} \mathrm{O}_{2} \mathrm{P}, 553.2045$; found, 553.2045.

$1 \mathrm{H}(\mathrm{CD} 2 \mathrm{Cl2}, 300 \mathrm{MHz}) \mathrm{Ph} 2 \mathrm{P}=\mathrm{O}$
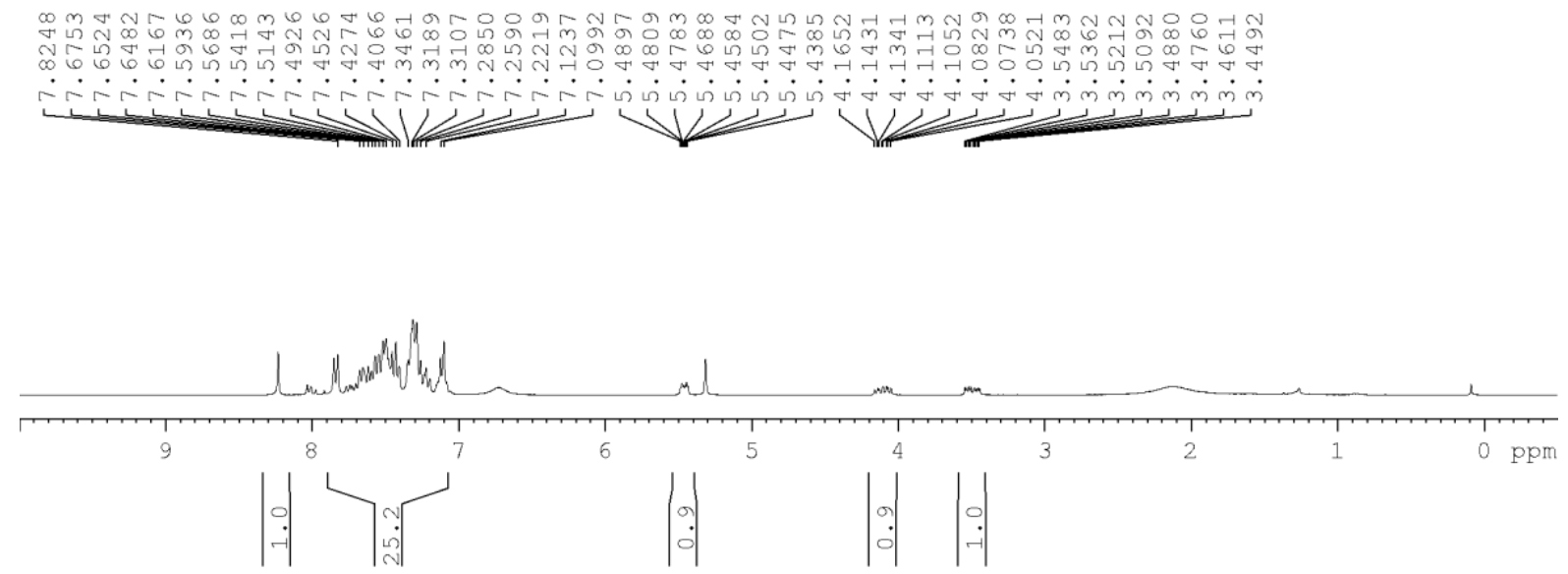

Figure S44: ${ }^{1} \mathrm{H}$ NMR spectrum of $3 \mathbf{e}$ 

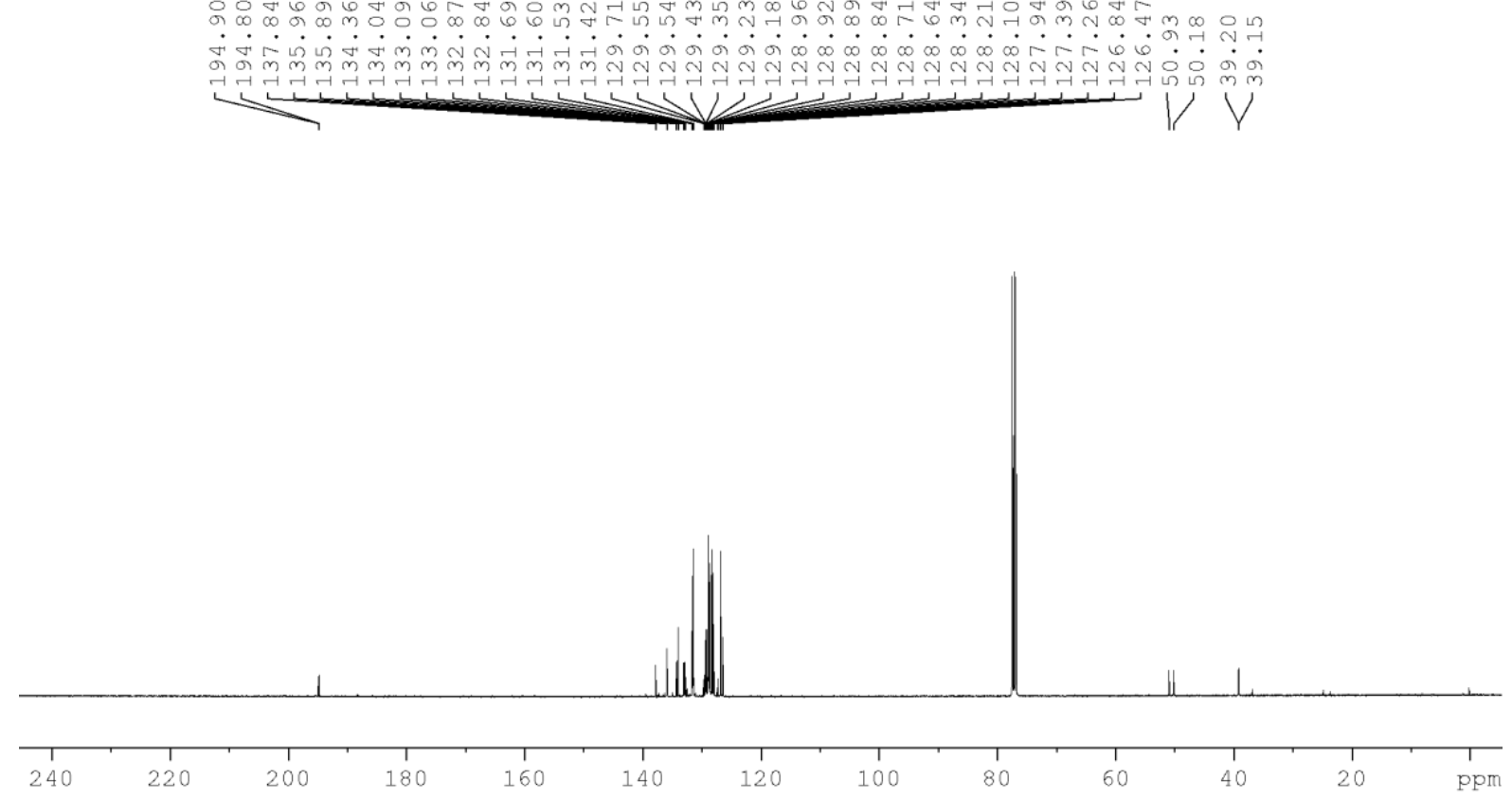

Figure S45: ${ }^{13} \mathrm{C}$ NMR spectrum of $\mathbf{3 e}$

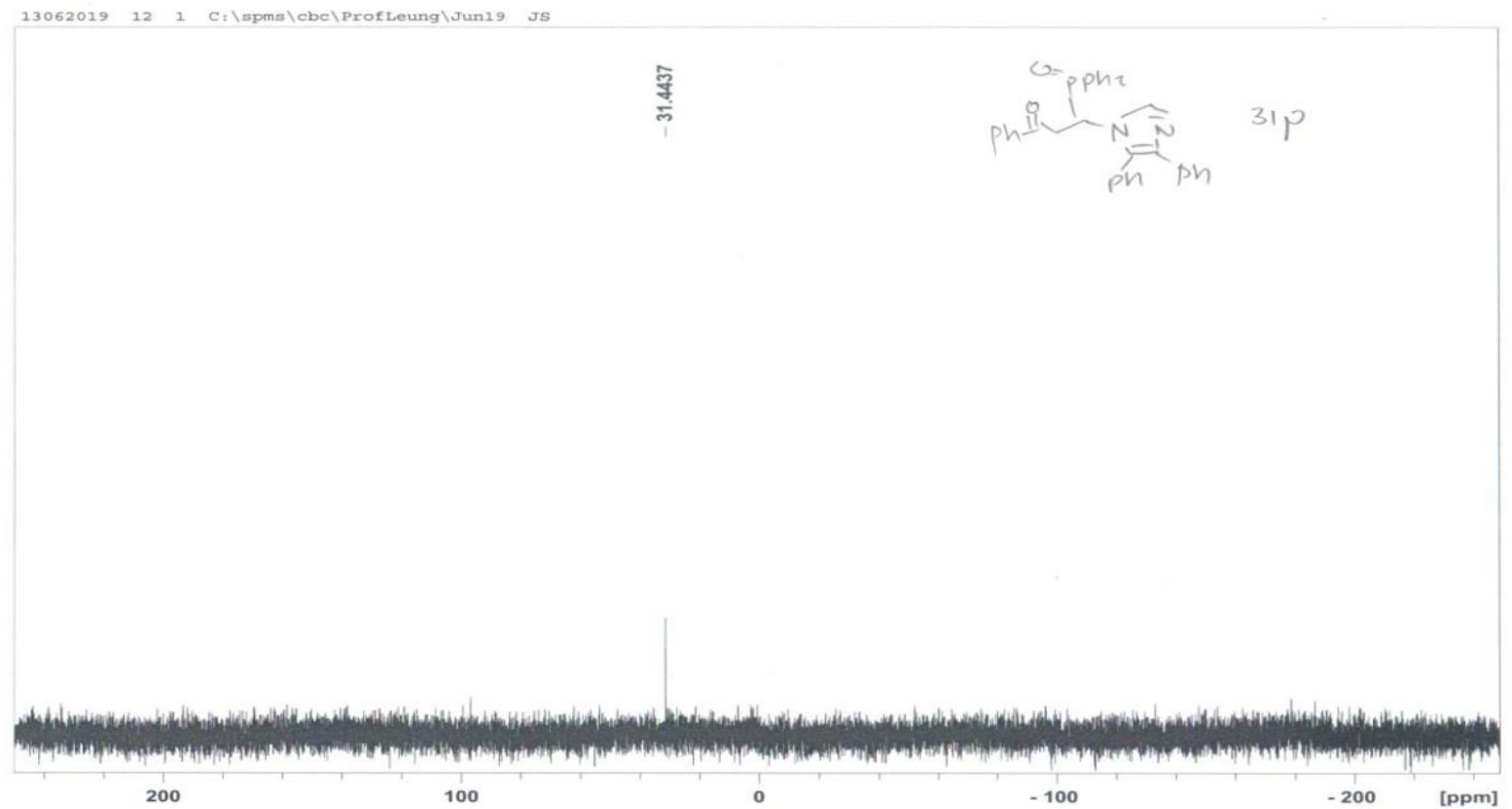

Figure S46: ${ }^{31} \mathrm{P}\left\{{ }^{1} \mathrm{H}\right\}$ NMR spectrum of $\mathbf{3 e}$ 
ID column, $1 \mathrm{~mL} / \mathrm{min}, 60$ Hexane: $40 \mathrm{i} \mathrm{PrOH}, 30^{\circ} \mathrm{C}$

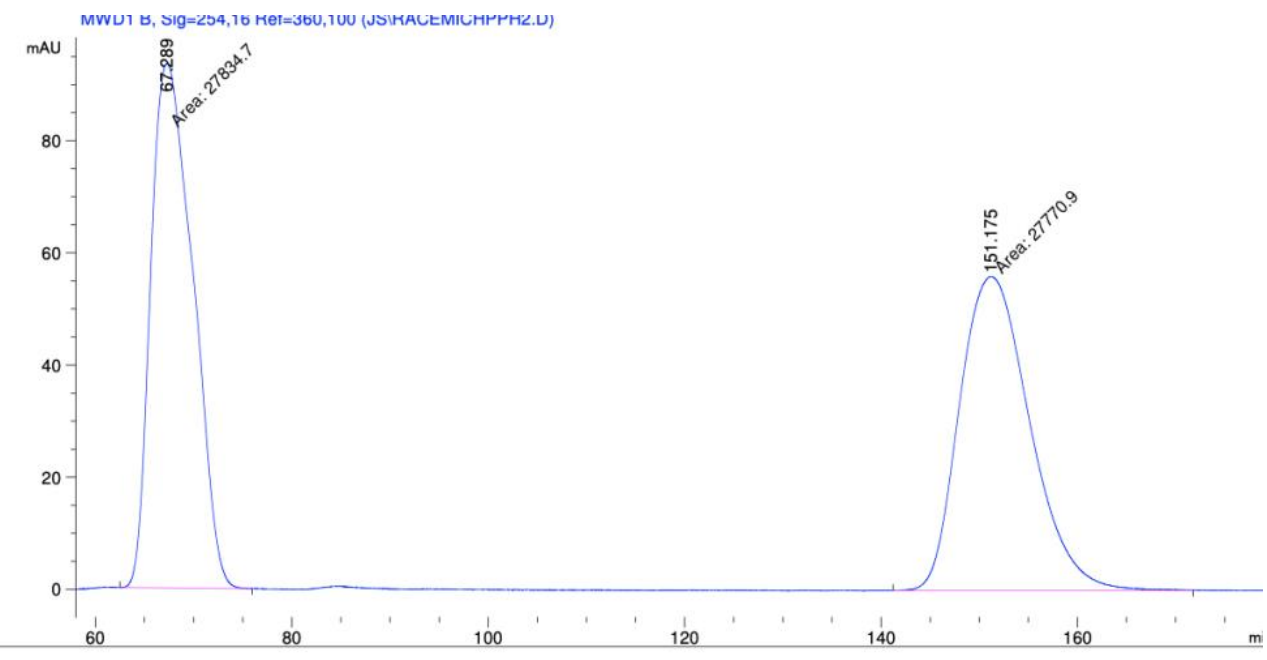

\begin{tabular}{|c|c|c|c|c|c|c|}
\hline $\begin{array}{c}\text { Peak } \\
\#\end{array}$ & $\begin{array}{l}\text { RetTime } \\
\text { [min] }\end{array}$ & Type & $\begin{array}{l}\text { Width } \\
\text { [min] }\end{array}$ & $\begin{array}{c}\text { Area } \\
{\left[\mathrm{mAU}^{\star} \mathrm{s}\right]}\end{array}$ & $\begin{array}{l}\text { Height } \\
\text { [MAU] }\end{array}$ & $\begin{array}{c}\text { Area } \\
\frac{\circ}{0}\end{array}$ \\
\hline 1 & 67.289 & MM T & 4.9627 & $2.78347 \mathrm{e} 4$ & 93.47956 & 50.0573 \\
\hline 2 & 151.175 & MM T & 8.2682 & $2.77709 \mathrm{e} 4$ & 55.97930 & 49.9427 \\
\hline ot & 10 & & & $5.56055 \mathrm{e} 4$ & 149.45886 & \\
\hline
\end{tabular}

Figure S47: Chiral HPLC spectrum of racemic 3e

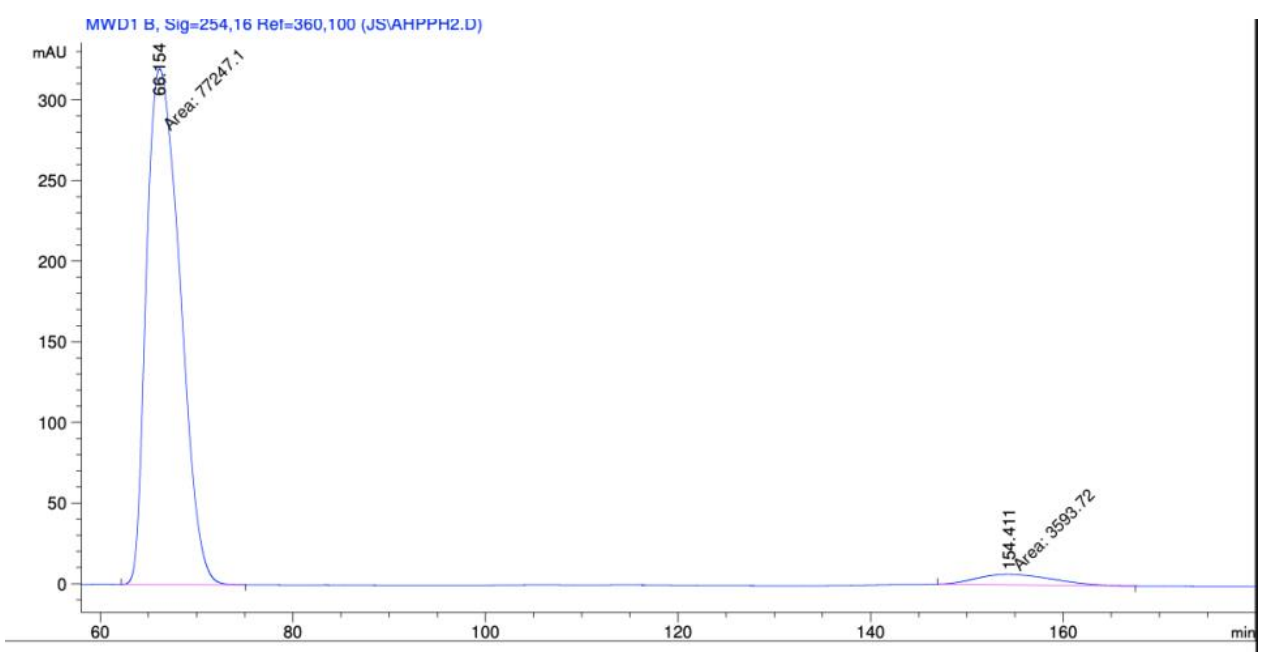

\begin{tabular}{|c|c|c|c|c|c|c|}
\hline eak & $\begin{array}{c}\text { RetTime } \\
\text { [min] }\end{array}$ & Type & $\begin{array}{c}\text { Width } \\
\text { [min] }\end{array}$ & $\begin{array}{c}\text { Area } \\
{\left[\mathrm{mAU}^{\star} \mathrm{s}\right]}\end{array}$ & $\begin{array}{l}\text { Height } \\
{[\mathrm{mAU}]}\end{array}$ & $\begin{array}{c}\text { Area } \\
\%\end{array}$ \\
\hline & & & & & & \\
\hline 1 & & $\mathbb{M} \mathrm{T}$ & 5 & $7.72471 \mathrm{e} 4$ & 319.90396 & 546 \\
\hline 2 & 154.411 & $\mathrm{M} \mathrm{T}$ & 9.1432 & 3593.72290 & 6.55082 & 4.4454 \\
\hline
\end{tabular}

Figure S48: Chiral HPLC spectrum of enantioenriched 3e 
<smiles>CCCCCCCCOC(=O)Cn1cnc(C)c1C</smiles>

Figure S49. Structure of $(S)-3 \mathbf{f}$

(S)-3f. White solid. Yield: $0.0081 \mathrm{~g}, 0.0190 \mathrm{mmol}, 33 \%$. $[\alpha]_{\mathrm{D}}\left(21{ }^{\circ} \mathrm{C}\right)=-3.1^{\circ} ;{ }^{1} \mathrm{H}$ NMR $\left(\mathrm{CD}_{2} \mathrm{Cl}_{2}, 300 \mathrm{MHz}\right): \delta 1.84$ (s, 3H, Me), 1.93 (s, 3H, Me), 3.42 (ddd, $1 \mathrm{H}, \mathrm{J}_{\mathrm{HH}}=18.3 \mathrm{~Hz}, 2.5$ $\left.\mathrm{Hz}, \mathrm{J}_{\mathrm{HP}}=7.1 \mathrm{~Hz}\right), 4.04\left(\mathrm{ddd}, 1 \mathrm{H}, \mathrm{J}_{\mathrm{HH}}=18.3 \mathrm{~Hz}, 10.3 \mathrm{~Hz}, \mathrm{~J}_{\mathrm{HP}}=4.4 \mathrm{~Hz}\right), 5.45\left(\mathrm{ddd}, 1 \mathrm{H}, \mathrm{J}_{\mathrm{HH}}=\right.$ $\left.10.2 \mathrm{~Hz}, 2.6 \mathrm{~Hz}, \mathrm{~J}_{\mathrm{HP}}=3.6 \mathrm{~Hz}\right), 7.35-7.62(\mathrm{~m}, 11 \mathrm{H}), 7.76-7.95(\mathrm{~m}, 5 \mathrm{H}) ;{ }^{13} \mathrm{C}\left\{{ }^{1} \mathrm{H}\right\} \mathrm{NMR}\left(\mathrm{CDCl}_{3}\right.$, $100 \mathrm{MHz}): \delta 7.94,11.0,39.4,50.6,51.3,124.8,127.2,128.3,128.9,129.0,129.5,129.6,130.7$ $(\mathrm{d}, \mathrm{J}=9.6 \mathrm{~Hz}), 131.4(\mathrm{~d}, \mathrm{~J}=8.6 \mathrm{~Hz}), 133.1,133.4,134.3,135.3,137.2,194.5(\mathrm{~d}, \mathrm{~J}=11.0 \mathrm{~Hz})$; ${ }^{31} \mathrm{P}\left\{{ }^{1} \mathrm{H}\right\}$ NMR $\left(\mathrm{CH}_{2} \mathrm{Cl}_{2}, 121 \mathrm{MHz}\right): \delta$ 34.18; HRMS (+ESI) $\mathrm{m} / \mathrm{z}:(\mathrm{M}+\mathrm{H})+$ calcd for $\mathrm{C}_{26} \mathrm{H}_{26} \mathrm{~N}_{2} \mathrm{O}_{2} \mathrm{P}, 429.1732$; found, 429.1736 .

$1 \mathrm{H}(\mathrm{CD} 2 \mathrm{Cl} 2,300) \mathrm{Me} 2 \mathrm{P}=\mathrm{O}$
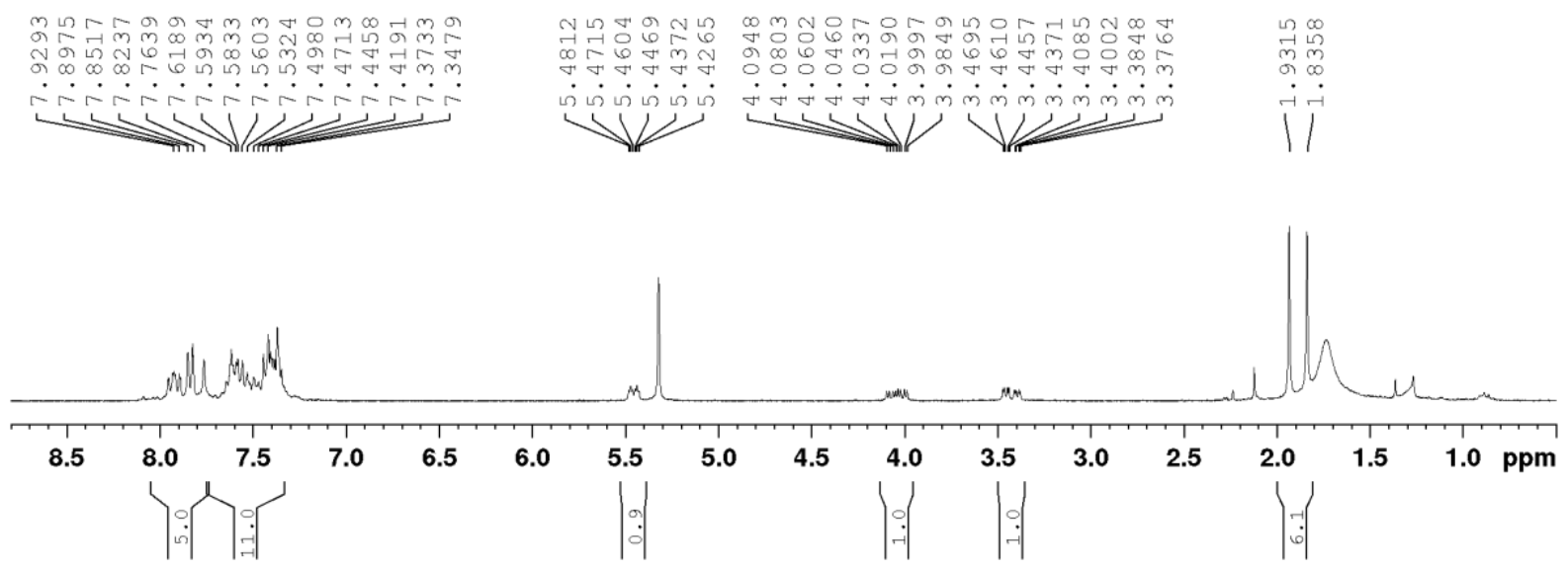

Figure S50: ${ }^{1} \mathrm{H}$ NMR spectrum of $\mathbf{3 f}$ 


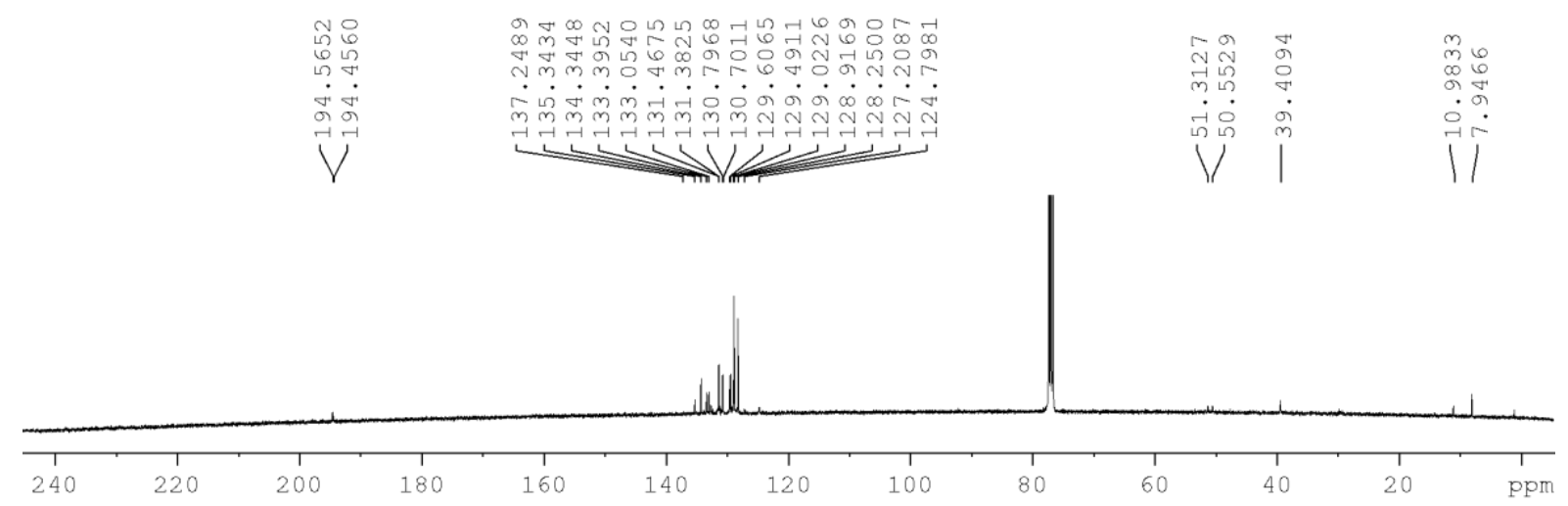

Figure S51: ${ }^{13} \mathrm{C}$ NMR spectrum of $\mathbf{3 f}$

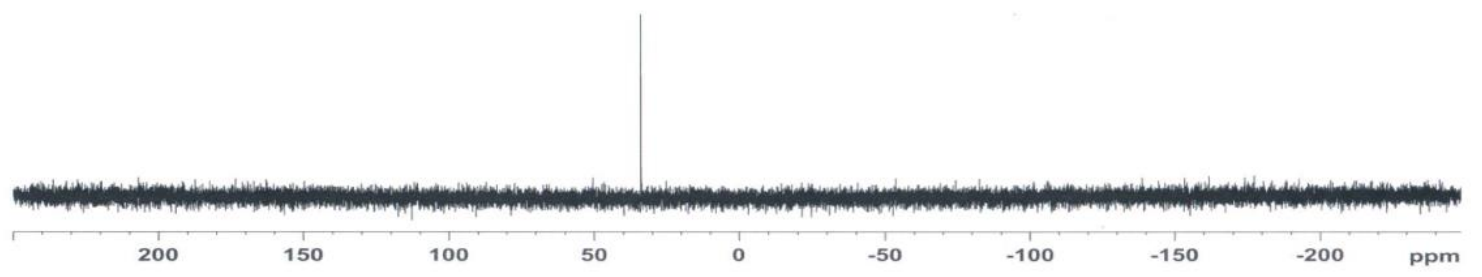

Figure S52: ${ }^{31} \mathrm{P}\left\{{ }^{1} \mathrm{H}\right\}$ NMR spectrum of $\mathbf{3 f}$ 
ID column, $1 \mathrm{~mL} / \mathrm{min}, 60$ Hexane: $40 \mathrm{i} \mathrm{PrOH}, 30^{\circ} \mathrm{C}$

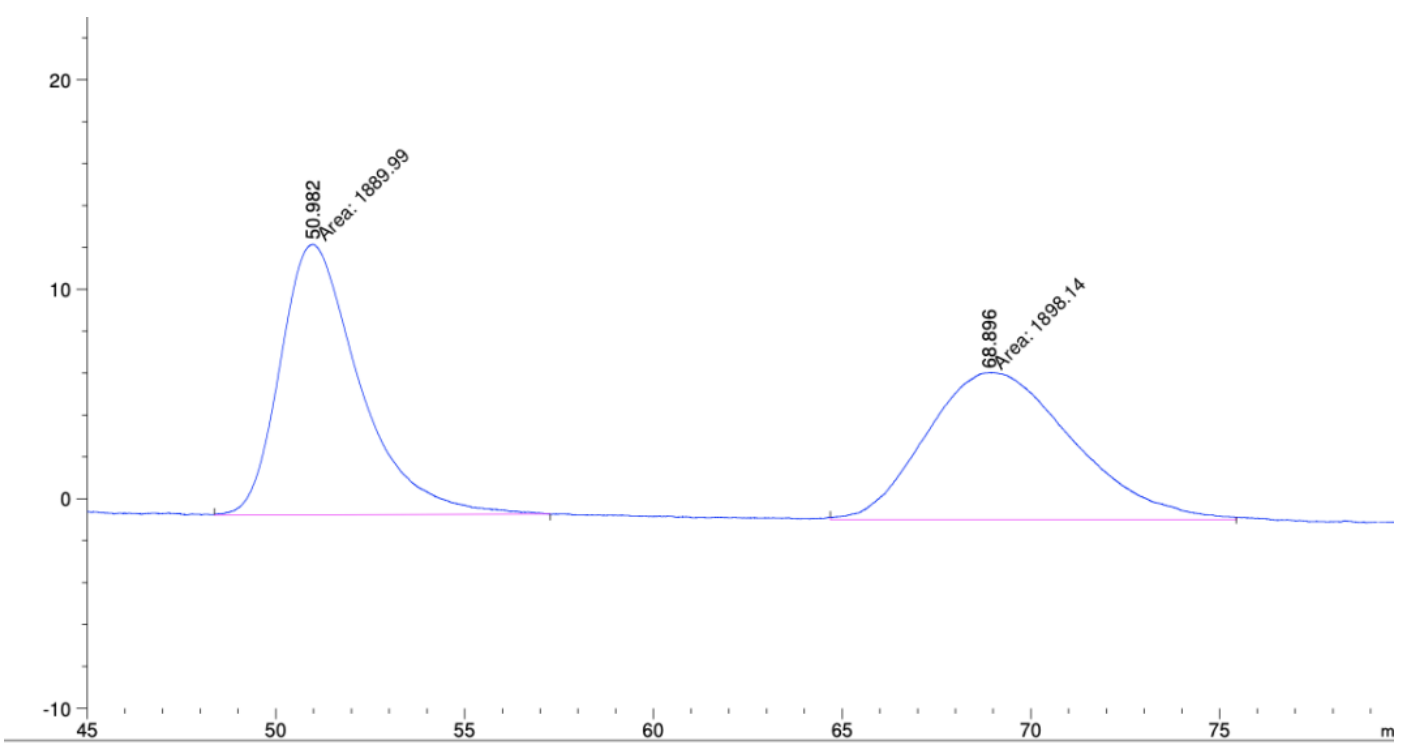

\begin{tabular}{|c|c|c|c|c|c|c|}
\hline $\begin{array}{c}\text { Peak } \\
\#\end{array}$ & $\begin{array}{c}\text { RetTime } \\
\text { [min] }\end{array}$ & Type & $\begin{array}{c}\text { Width } \\
\text { [min] }\end{array}$ & $\begin{array}{c}\text { Area } \\
{\left[\mathrm{mAU}^{\star} \mathrm{s}\right]}\end{array}$ & $\begin{array}{l}\text { Height } \\
\text { [mAU] }\end{array}$ & $\begin{array}{c}\text { Area } \\
\frac{\circ}{0}\end{array}$ \\
\hline--1 & 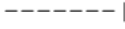 & 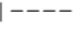 & -- & -------- & ------- & -- \\
\hline 1 & 50.982 & MM T & 2.4390 & 1889.98608 & 12.91510 & 49.8923 \\
\hline 2 & 68.896 & $\mathrm{MM} \mathrm{T}$ & 4.5065 & 1898.14355 & 7.02007 & 50.1077 \\
\hline Total & : & & & 3788.12964 & 19.93517 & \\
\hline
\end{tabular}

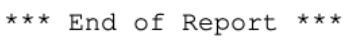

Figure S53: Chiral HPLC spectrum of racemic 3f 


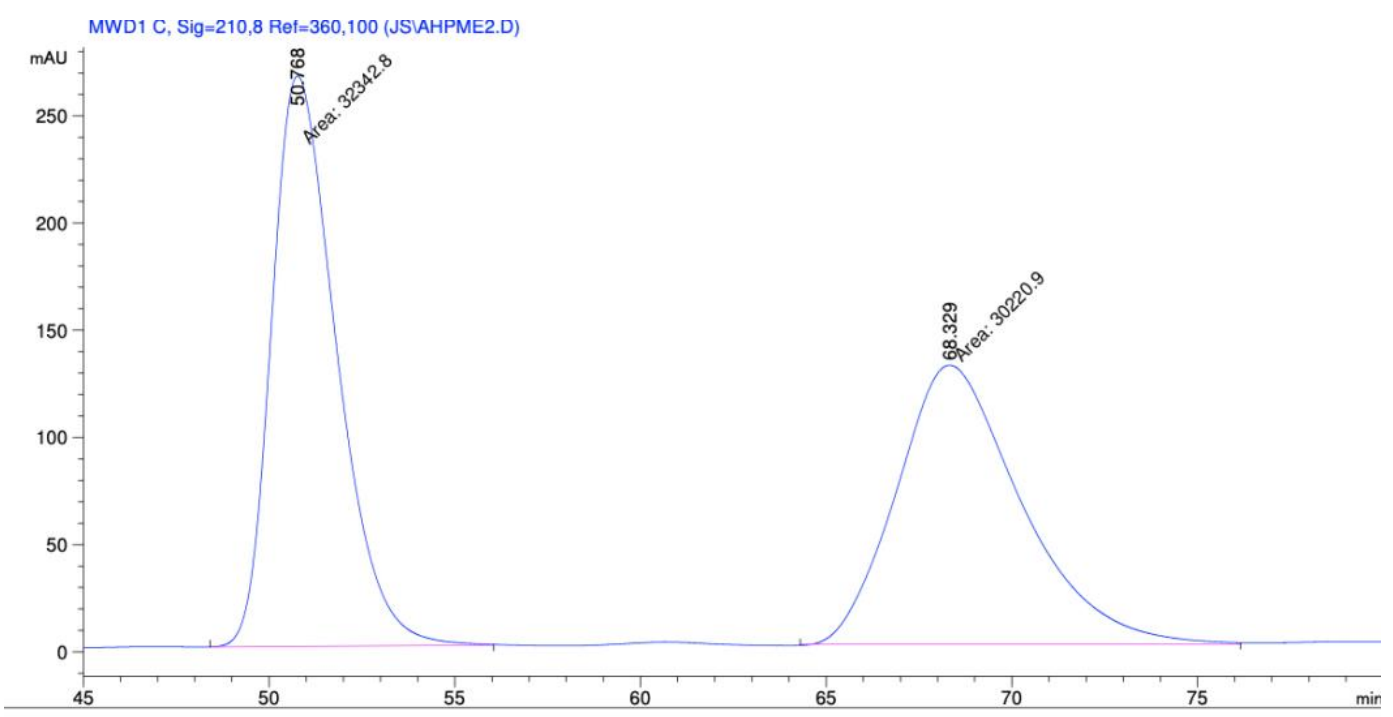

Signal 1: MWD1 C, Sig=210,8 Ref $=360,100$

\begin{tabular}{|c|c|c|c|c|c|c|}
\hline $\begin{array}{c}\text { eak } \\
\#\end{array}$ & $\begin{array}{c}\text { RetTime } \\
\text { [min] }\end{array}$ & Type & $\begin{array}{c}\text { Width } \\
\text { [min] }\end{array}$ & $\begin{array}{c}\text { Area } \\
{\left[\mathrm{mAU}{ }^{\star} \mathrm{s}\right]}\end{array}$ & $\begin{array}{l}\text { Height } \\
{[\mathrm{mAU}]}\end{array}$ & $\begin{array}{c}\text { Area } \\
\frac{\circ}{\delta}\end{array}$ \\
\hline I & & & & & & \\
\hline & 68 & $M T$ & 7 & $02209 e 4$ & 130.05841 & 3042 \\
\hline
\end{tabular}

Figure S54: Chiral HPLC spectrum of enantioenriched $3 f$ 
<smiles>O=C(CCn1cnc2ccccc21)c1ccccc1</smiles>

Figure S55. Structure of $(S)-3 g$

(S)-3g. White solid. Yield: $0.0210 \mathrm{~g}, 0.0466 \mathrm{mmol}, 81 \%$. $[\alpha]_{\mathrm{D}}\left(21{ }^{\circ} \mathrm{C}\right)=7.6^{\circ} ;{ }^{1} \mathrm{H}$ NMR $\left(\mathrm{CD}_{2} \mathrm{Cl}_{2}, 300 \mathrm{MHz}\right): \delta 3.61\left(\mathrm{ddd}, 1 \mathrm{H}, \mathrm{J}_{\mathrm{HH}}=18.5 \mathrm{~Hz}, 2.3 \mathrm{~Hz}, \mathrm{~J}_{\mathrm{HP}}=7.5 \mathrm{~Hz}\right), 4.19$ (ddd, $1 \mathrm{H}, \mathrm{J}_{\mathrm{HH}}$ $\left.=18.5 \mathrm{~Hz}, 9.7 \mathrm{~Hz}, \mathrm{~J}_{\mathrm{HP}}=4.2 \mathrm{~Hz}\right), 6.09\left(\mathrm{ddd}, 1 \mathrm{H}, \mathrm{J}_{\mathrm{HH}}=9.5 \mathrm{~Hz}, 2.4 \mathrm{~Hz}, \mathrm{~J}_{\mathrm{HP}}=4.0 \mathrm{~Hz}\right), 7.13-7.41$ $(\mathrm{m}, 7 \mathrm{H}), 7.50-7.65(\mathrm{~m}, 7 \mathrm{H}), 7.74-7.83(\mathrm{~m}, 3 \mathrm{H}), 8.02(\mathrm{t}, 2 \mathrm{H}, \mathrm{J} \mathrm{HH}=9.3 \mathrm{~Hz}), 8.28(\mathrm{~s}, 1 \mathrm{H}) ;{ }^{13} \mathrm{C}\left\{{ }^{1} \mathrm{H}\right\}$ NMR $\left(\mathrm{CDCl}_{3}, 100 \mathrm{MHz}\right): \delta 38.3,50.3,51.1,110.8,120.3,122.4,122.8,123.3,128.3,128.7$ $(\mathrm{d}, \mathrm{J}=11.9 \mathrm{~Hz}), 128.9,129.5(\mathrm{~d}, \mathrm{~J}=11.6 \mathrm{~Hz}), 130.6(\mathrm{~d}, \mathrm{~J}=9.7 \mathrm{~Hz}), 131.4(\mathrm{~d}, \mathrm{~J}=8.9 \mathrm{~Hz})$, 132.7 (d, J = 2.6 Hz), 133.1 (d, J = 2.3 Hz), 133.8, 134.1, 135.6, 142.7, 143.1, 194.9 (d, J = $10.3 \mathrm{~Hz}) ;{ }^{31} \mathrm{P}\left\{{ }^{1} \mathrm{H}\right\} \mathrm{NMR}\left(\mathrm{CH}_{2} \mathrm{Cl}_{2}, 121 \mathrm{MHz}\right): \delta 31.32$; HRMS (+ESI) m/z: $(\mathrm{M}+\mathrm{H})+$ calcd for $\mathrm{C}_{28} \mathrm{H}_{24} \mathrm{~N}_{2} \mathrm{O}_{2} \mathrm{P}, 451.1575$; found, 451.1575.

$1 \mathrm{H}(\mathrm{CD} 2 \mathrm{Cl2}, 300 \mathrm{MHz}) \mathrm{Bn} \mathrm{P}=\mathrm{O}$

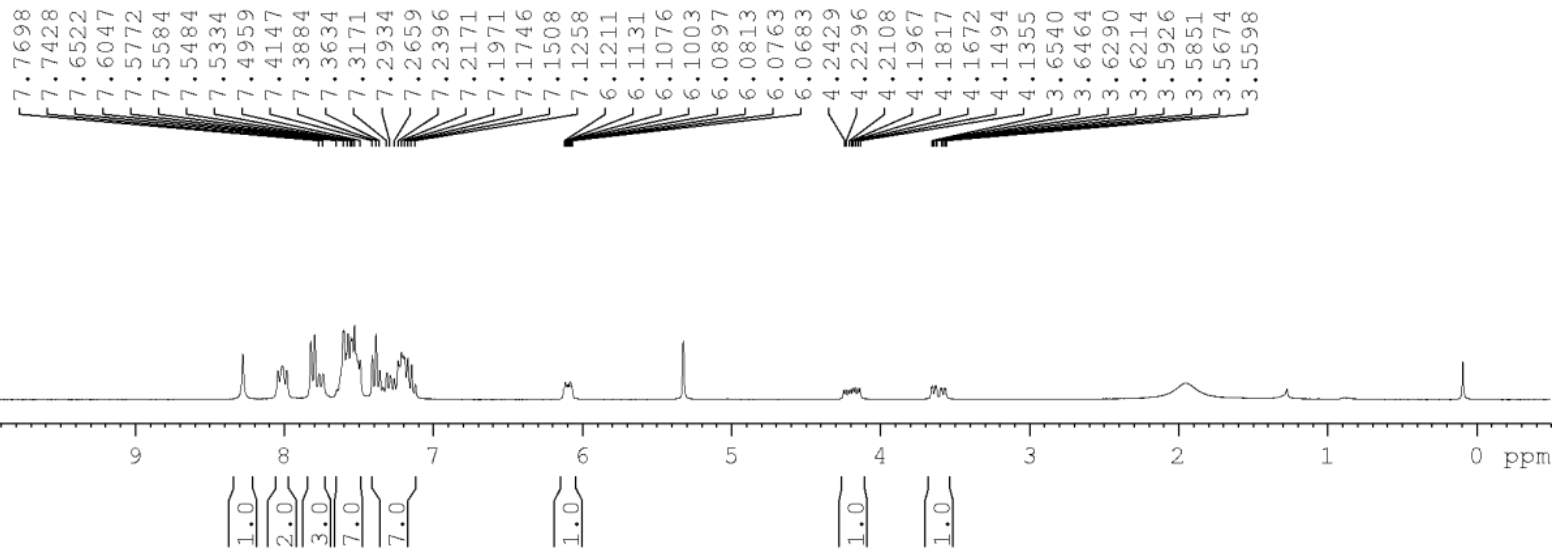

Figure S56: ${ }^{1} \mathrm{H}$ NMR spectrum of $\mathbf{3 g}$ 


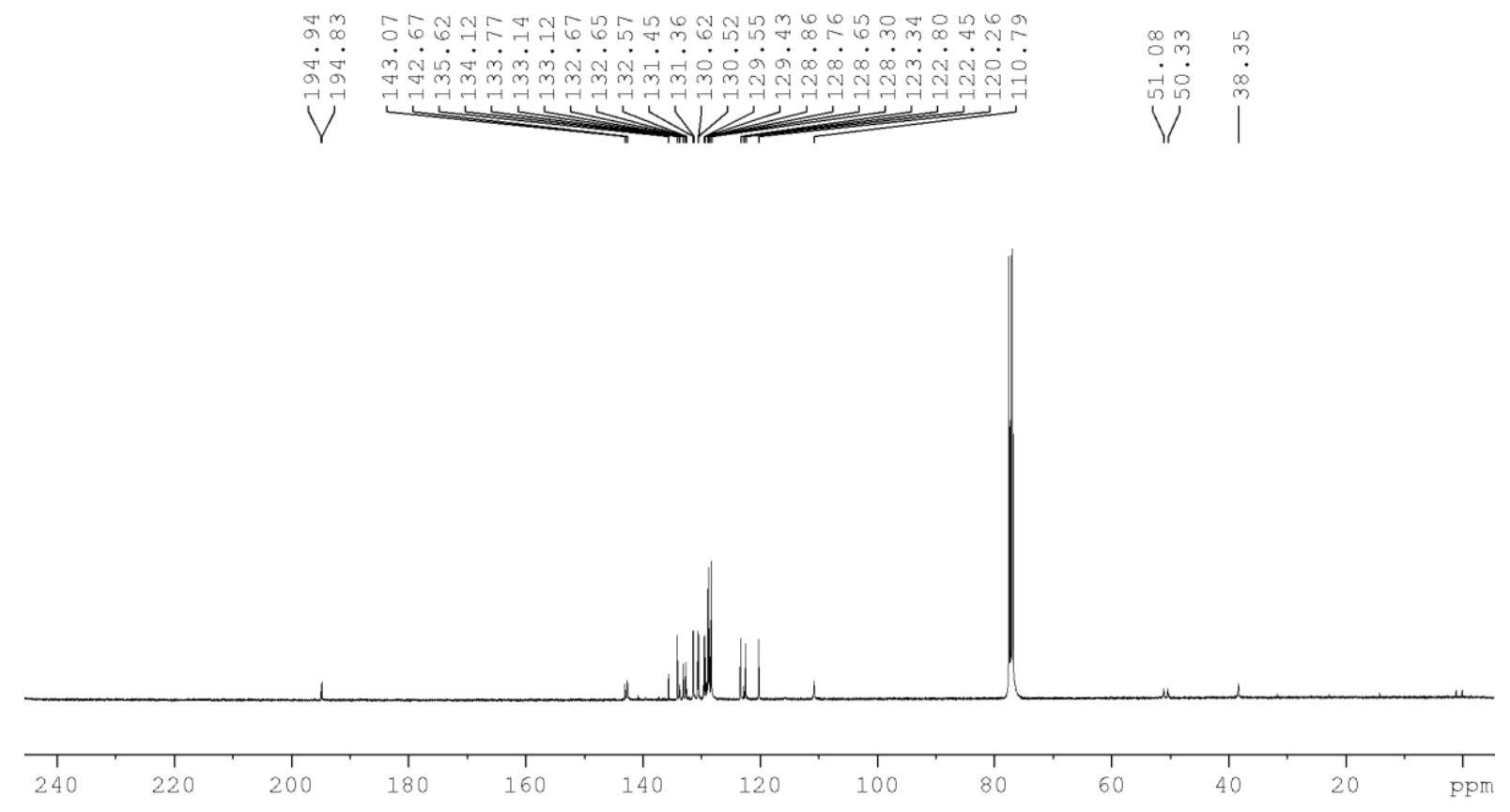

Figure S57: ${ }^{13} \mathrm{C}$ NMR spectrum of $\mathbf{3 g}$

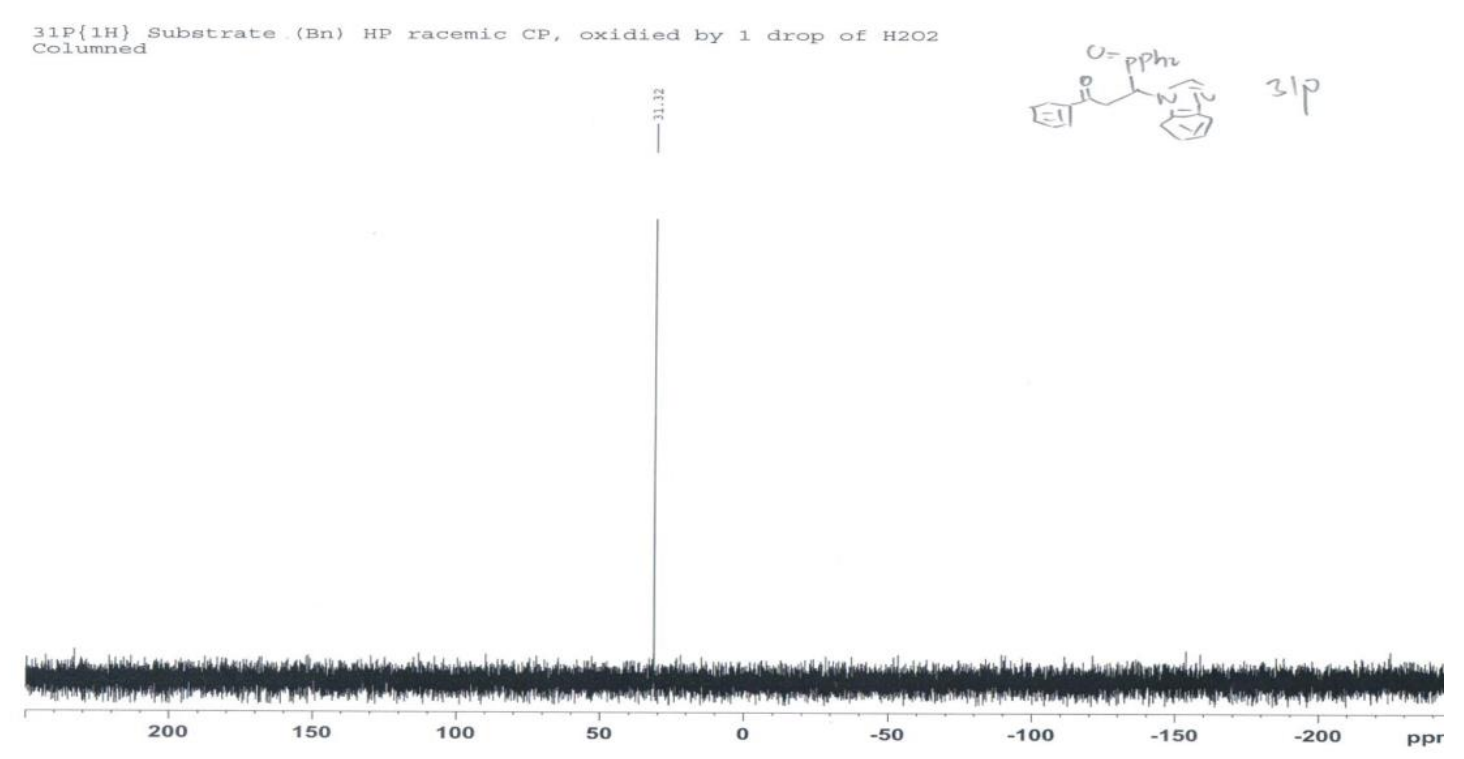

Figure S58: ${ }^{31} \mathrm{P}\left\{{ }^{1} \mathrm{H}\right\}$ NMR spectrum of $\mathbf{3 g}$ 
ID column, $1 \mathrm{~mL} / \mathrm{min}, 60$ Hexane: $40 \mathrm{i} \mathrm{PrOH}, 30^{\circ} \mathrm{C}$
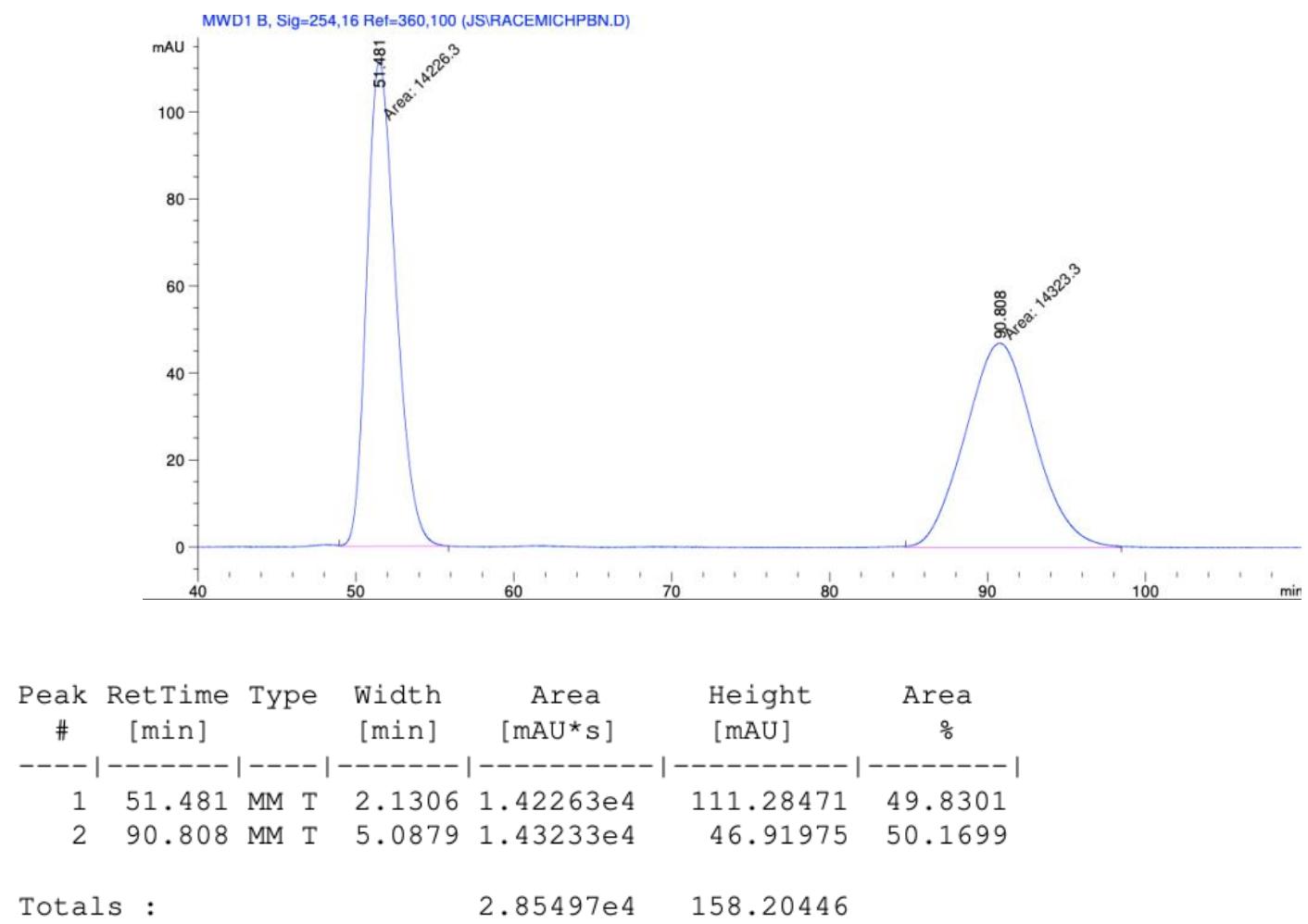

Figure S59: Chiral HPLC spectrum of racemic 3g
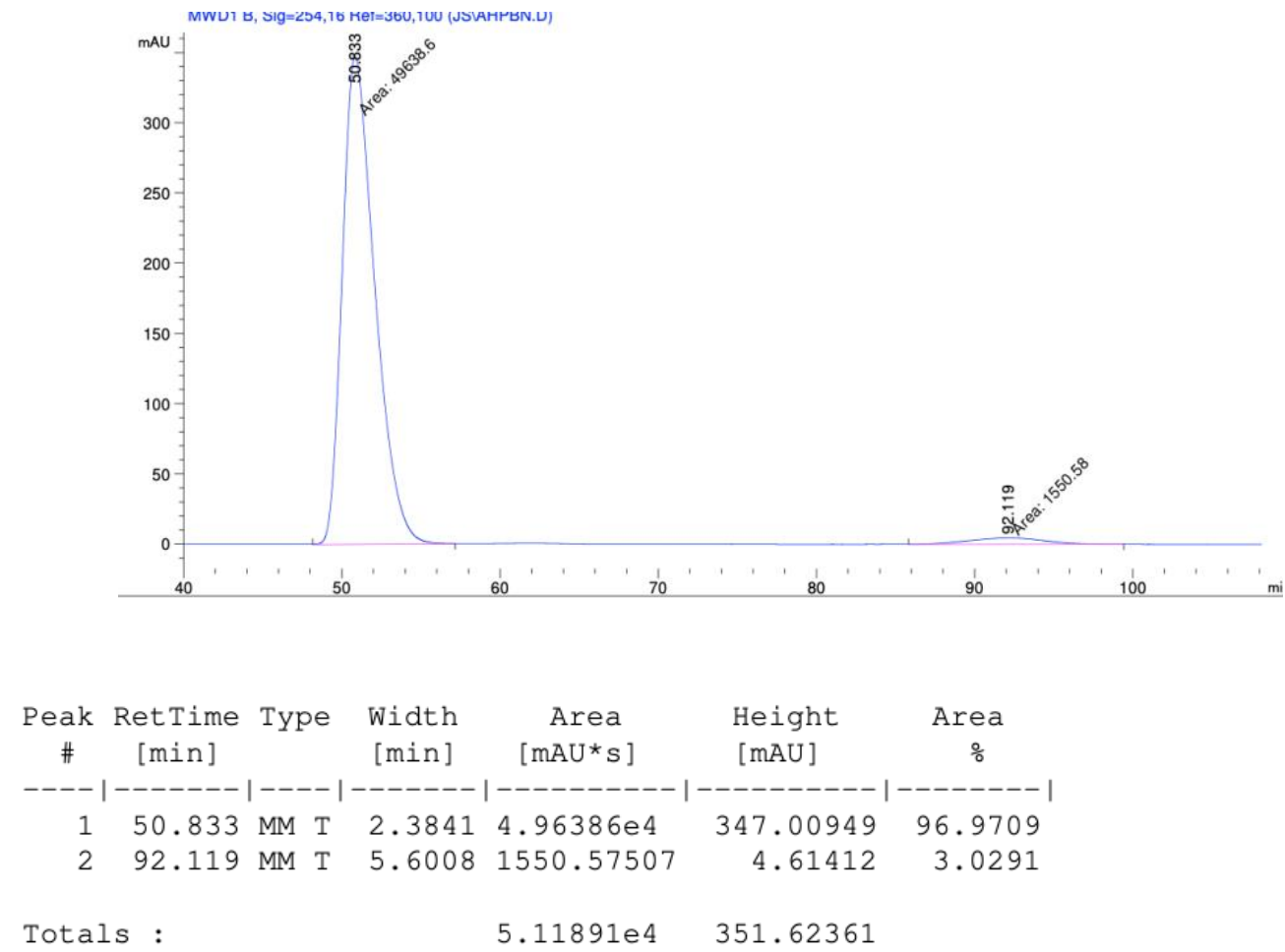

Figure S60: Chiral HPLC spectrum of enantioenriched 3g 


\section{General synthesis of complex $(S)-5 g$}

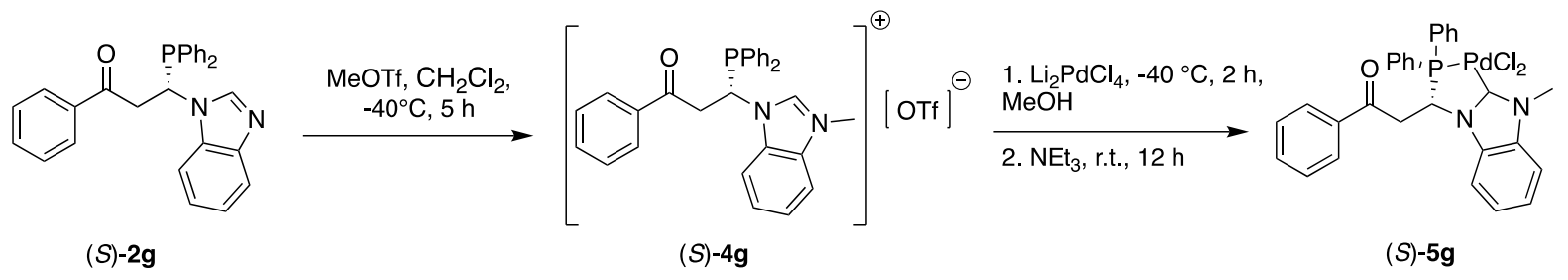

Scheme S6: Synthesis of $(S)-5 g$.

Diphenylphosphine (10 uL, $0.0575 \mathrm{mmol}, 1$ equiv), $\mathrm{CH}_{2} \mathrm{Cl}_{2}(1150 \mathrm{uL})$ and $(R)-\mathbf{C 3}(0.0009 \mathrm{~g}$, $2.5 \mathrm{~mol} \%$ ) were added to an oven-dried $10 \mathrm{~mL}$ storage tube and cooled to $-78^{\circ} \mathrm{C}$. Substrate $1 \mathrm{~g}(0.0575 \mathrm{mmol}, 1$ equiv) and tetramethylethylenediamine $(8.6 \mathrm{uL}, 1$ equiv) were added sequentially into the mixture and the reaction mixture was stirred at $-78{ }^{\circ} \mathrm{C}$ for the stipulated time. After which, the reaction mixture was filtered through a short plug of celite to give $(S)$ 2g. Solvent and base were fully removed by heating the mixture to $50{ }^{\circ} \mathrm{C}$ under strong vacuum for $1 \mathrm{hr}$. $\mathrm{CH}_{2} \mathrm{Cl}_{2}(1150 \mathrm{uL})$ was then added and the reaction mixture was cooled back to $-40{ }^{\circ} \mathrm{C}$. MeOTf $(6.5 \mathrm{uL}, 0.0590 \mathrm{mmol})$ was added and the mixture was left to stir for $5 \mathrm{hrs}$ to give $(S)$ $\mathbf{4 g}$ which was not isolated due to difficulty in purifying the air sensitive sticky compound. A methanolic solution of $\mathrm{Li}_{2} \mathrm{PdCl}_{4}(0.0575 \mathrm{mmol}, 1$ equiv. $)$ was subsequently added and the solution was left to stir for another $2 \mathrm{hrs}$ before triethylamine ( $8 \mathrm{uL}, 1$ equiv) was added. Finally, the mixture was warmed up to room temperature and stirred overnight. Volatiles were removed under reduced pressure and the crude mixture was purified via repeated recrystallizations $\left(\mathrm{CH}_{2} \mathrm{Cl}_{2} /\right.$ hexane) to afford the enantiopure $(S)-5 \mathrm{~g}$. Complex $(S)-\mathbf{5 g}$ was observed to decompose with significant loss in yield on silica gel and was hence not purified by column chromatography.

\section{Characterization of complex $(S)-5 g$}

(S)-5g. Pale yellow solid. Yield: $0.0065 \mathrm{~g}, 18 \%$. $[\alpha]_{\mathrm{D}}\left(2{ }^{\circ} \mathrm{C}\right)=209.3^{\circ} ;{ }^{1} \mathrm{H}$ NMR $\left(\mathrm{CD}_{2} \mathrm{Cl}_{2}, 500\right.$ MHz): $\delta 3.45(\mathrm{td}, 1 \mathrm{H}, \mathrm{J}=27.9 \mathrm{~Hz}, 2.9 \mathrm{~Hz}), 4.02-4.10(\mathrm{~m}, 1 \mathrm{H}), 4.47$ (s, 3H, Me), 6.21-6.26 (m, $1 \mathrm{H}), 7.16\left(\mathrm{t}, 3 \mathrm{H}, \mathrm{J}_{\mathrm{HH}}=2.4 \mathrm{~Hz}\right), 7.35\left(\mathrm{t}, 2 \mathrm{H}, \mathrm{J}_{\mathrm{HH}}=7.9 \mathrm{~Hz}\right), 7.43-7.48(\mathrm{~m}, 2 \mathrm{H}), 7.53-7.62(\mathrm{~m}$, $8 \mathrm{H}), 7.83-7.87(\mathrm{~m}, 2 \mathrm{H}), 8.18-8.22(\mathrm{~m}, 2 \mathrm{H}) ;{ }^{13} \mathrm{C}\left\{{ }^{1} \mathrm{H}\right\} \mathrm{NMR}\left(\mathrm{CD}_{2} \mathrm{Cl}_{2}, 75 \mathrm{MHz}\right): \delta 36.0,51.2(\mathrm{~d}$, $\mathrm{J}=6.5 \mathrm{~Hz}), 111.3,112.1,123.8,124.6(\mathrm{~d}, \mathrm{~J}=4.8 \mathrm{~Hz}), 125.1,125.3,127.9,128.2,128.4,128.5$, $129.6(\mathrm{~d}, \mathrm{~J}=11.4 \mathrm{~Hz}), 130.8(\mathrm{~d}, \mathrm{~J}=11.7 \mathrm{~Hz}), 132.7$ (d, J = 13.0 Hz), 133.7 (d, J = 11.3 Hz), 134.1, 134.8, 135.8, 135.9, 135.9, 172.1, 194.4 (d, J = 1.8 Hz); ${ }^{31} \mathrm{P}\left\{{ }^{1} \mathrm{H}\right\} \mathrm{NMR}\left(\mathrm{CD}_{2} \mathrm{Cl}_{2}, 202\right.$ $\mathrm{MHz}): \delta 59.2$. 


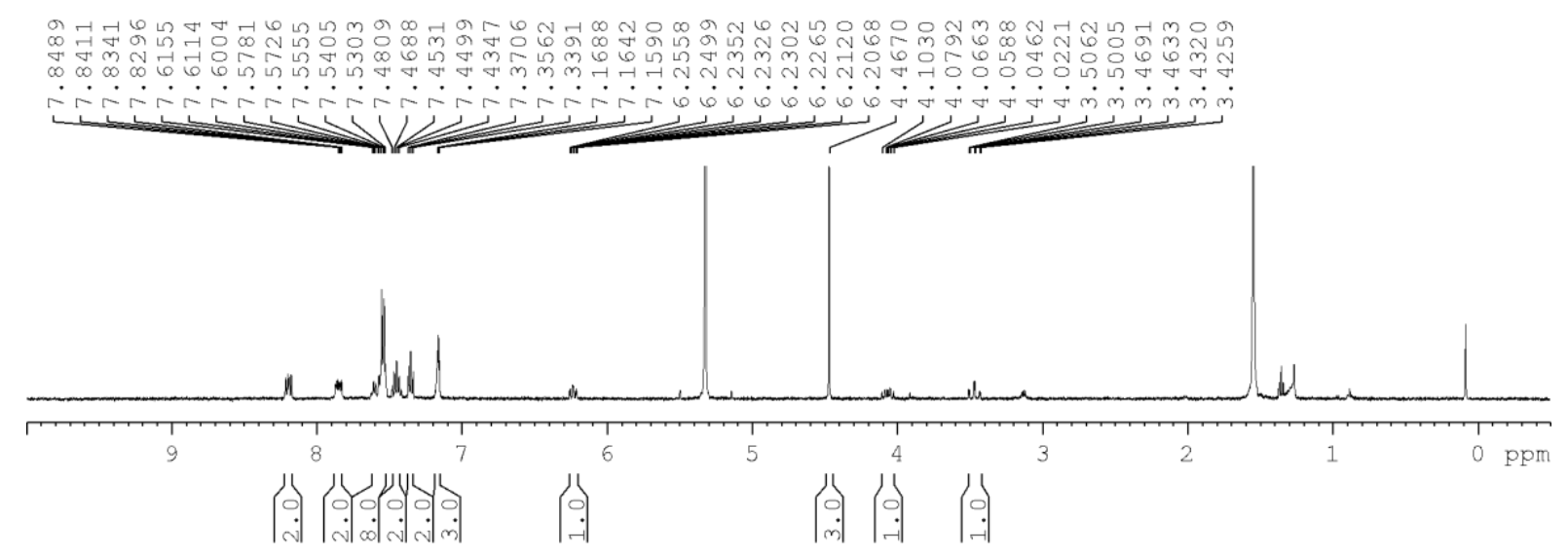

Figure S61: ${ }^{1} \mathrm{H}$ NMR spectrum of $\mathbf{5 g}$

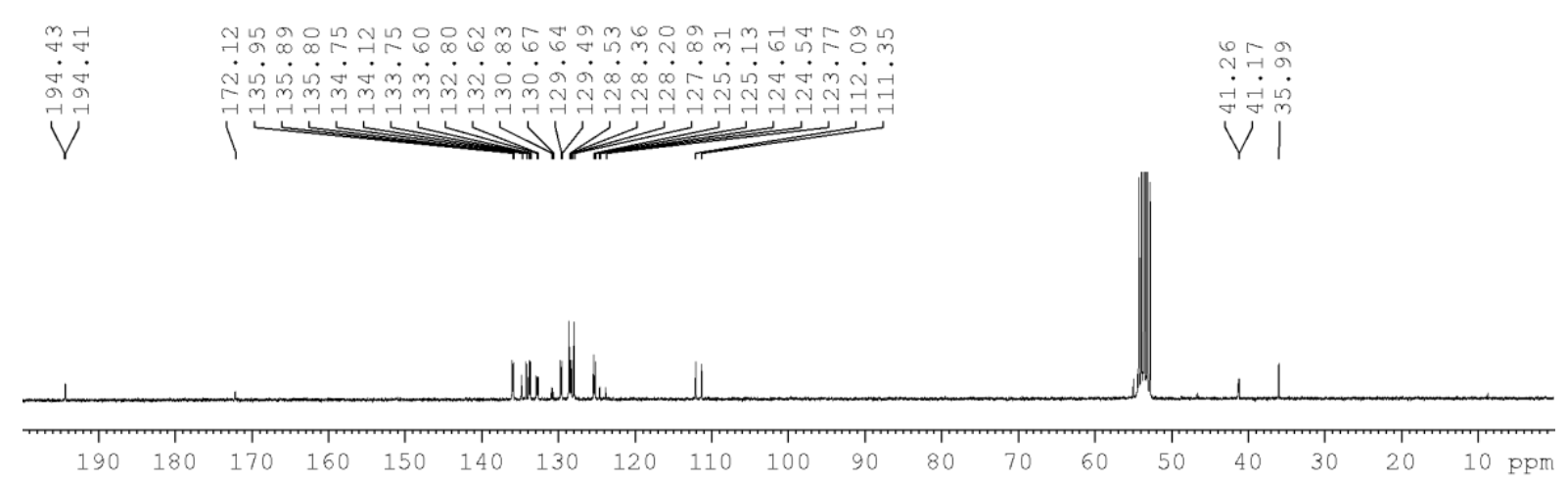

Figure S62: ${ }^{13} \mathrm{C}$ NMR spectrum of $\mathbf{5 g}$

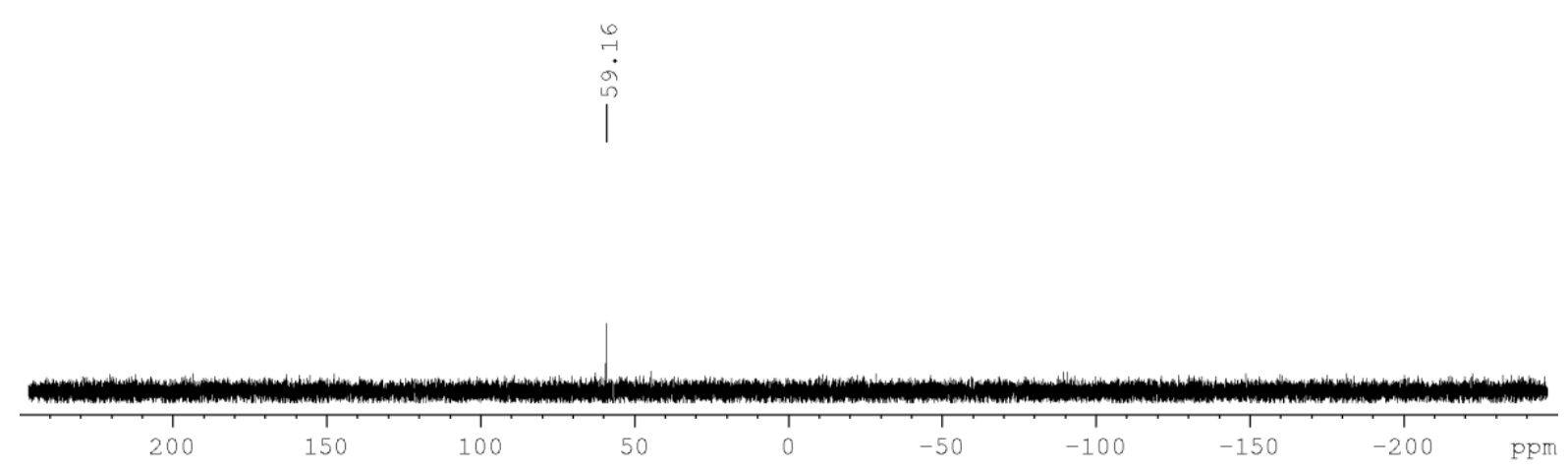

Figure S63: ${ }^{31} \mathrm{P}\left\{{ }^{1} \mathrm{H}\right\}$ NMR spectrum of $\mathbf{5 g}$ 


\section{Crystallographic Information}

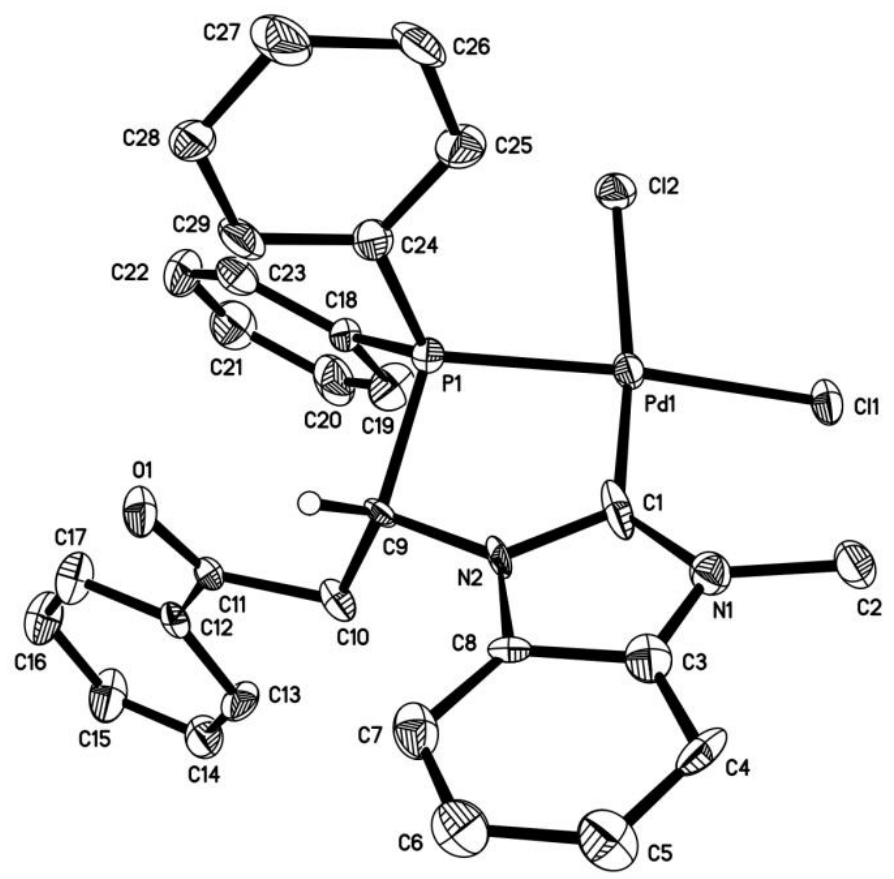

Figure S64. Molecular structure of chiral chelating P-NHC Pd(II) chloride complex $(S)-5 g$ with thermal ellipsoids shown at 50\% probability (CCDC Ref.: 2035450). Hydrogen atoms except $\mathrm{H}(\mathrm{C} 9)$ are omitted for clarity

\section{A) Crystal Data}

Chemical formula

Formula weight

Temperature

Wavelength

Crystal size

Crystal habit

Crystal system

Space group

Unit cell dimensions

\section{Volume}

Z

Density (calculated)

Absorption coefficient

F(000)
$\mathrm{C}_{29} \mathrm{H}_{25} \mathrm{Cl}_{2} \mathrm{~N}_{2} \mathrm{OPPd}$

$625.78 \mathrm{~g} / \mathrm{mol}$

100(2) K

$0.71073 \AA$

$0.100 \times 0.140 \times 0.200 \mathrm{~mm}$

yellow block

monoclinic

P 1211

$a=15.573(2) \AA \quad \alpha=90^{\circ}$

$\mathrm{b}=12.0833(16) \AA \quad \beta=90.106(3)^{\circ}$

$\mathrm{c}=20.842(3) \AA \quad \gamma=90^{\circ}$

3921.9(9) $\AA^{3}$

6

$1.590 \mathrm{~g} / \mathrm{cm}^{3}$

$1.002 \mathrm{~mm}^{-1}$

1896 


\section{B) Bond lengths}

\begin{tabular}{|c|c|c|c|}
\hline $\mathrm{C} 1-\mathrm{N} 2$ & $1.39(2)$ & $\mathrm{C} 1-\mathrm{N} 1$ & $1.39(3)$ \\
\hline $\mathrm{C} 1-\mathrm{Pd} 1$ & $1.93(2)$ & $\mathrm{C} 2-\mathrm{N} 1$ & $1.45(2)$ \\
\hline $\mathrm{C} 2-\mathrm{H} 2 \mathrm{~A}$ & 0.98 & $\mathrm{C} 2-\mathrm{H} 2 \mathrm{~B}$ & 0.98 \\
\hline $\mathrm{C} 2-\mathrm{H} 2 \mathrm{C}$ & 0.98 & $\mathrm{C} 3-\mathrm{N} 1$ & $1.370(18)$ \\
\hline $\mathrm{C} 3-\mathrm{C} 4$ & 1.39 & $\mathrm{C} 3-\mathrm{C} 8$ & 1.39 \\
\hline $\mathrm{C} 4-\mathrm{C} 5$ & 1.39 & $\mathrm{C} 4-\mathrm{H} 4$ & 0.95 \\
\hline C5-C6 & 1.39 & $\mathrm{C} 5-\mathrm{H} 5$ & 0.95 \\
\hline C6-C7 & 1.39 & C6-H6 & 0.95 \\
\hline C7-C8 & 1.39 & $\mathrm{C} 7-\mathrm{H} 7$ & 0.95 \\
\hline $\mathrm{C} 8-\mathrm{N} 2$ & $1.364(18)$ & $\mathrm{C} 9-\mathrm{N} 2$ & $1.46(2)$ \\
\hline C9-C10 & $1.52(2)$ & C9-P1 & $1.864(18)$ \\
\hline C9-H9 & 1.0 & C10-C11 & $1.51(3)$ \\
\hline C10-H10A & 0.99 & C10-H10B & 0.99 \\
\hline $\mathrm{C} 11-\mathrm{O} 1$ & $1.21(2)$ & C11-C12 & $1.50(2)$ \\
\hline C12-C13 & 1.39 & C12-C17 & 1.39 \\
\hline C13-C14 & 1.39 & C13-H13 & 0.95 \\
\hline C14-C15 & 1.39 & C14-H14 & 0.95 \\
\hline C15-C16 & 1.39 & $\mathrm{C} 15-\mathrm{H} 15$ & 0.95 \\
\hline C16-C17 & 1.39 & C16-H16 & 0.95 \\
\hline C17-H17 & 0.95 & C18-C19 & 1.39 \\
\hline $\mathrm{C} 18-\mathrm{C} 23$ & 1.39 & C18-P1 & $1.804(11)$ \\
\hline C19-C20 & 1.39 & C19-H19 & 0.95 \\
\hline C20-C21 & 1.39 & $\mathrm{C} 20-\mathrm{H} 20$ & 0.95 \\
\hline $\mathrm{C} 21-\mathrm{C} 22$ & 1.39 & $\mathrm{C} 21-\mathrm{H} 21$ & 0.95 \\
\hline $\mathrm{C} 22-\mathrm{C} 23$ & 1.39 & $\mathrm{C} 22-\mathrm{H} 22$ & 0.95 \\
\hline $\mathrm{C} 23-\mathrm{H} 23$ & 0.95 & $\mathrm{C} 24-\mathrm{C} 25$ & 1.39 \\
\hline C24-C29 & 1.39 & C24-P1 & $1.810(9)$ \\
\hline $\mathrm{C} 25-\mathrm{C} 26$ & 1.39 & $\mathrm{C} 25-\mathrm{H} 25$ & 0.95 \\
\hline C26-C27 & 1.39 & C26-H26 & 0.95 \\
\hline C27-C28 & 1.39 & $\mathrm{C} 27-\mathrm{H} 27$ & 0.95 \\
\hline C28-C29 & 1.39 & $\mathrm{C} 28-\mathrm{H} 28$ & 0.95 \\
\hline C29-H29 & 0.95 & $\mathrm{C} 30-\mathrm{N} 3$ & $1.34(2)$ \\
\hline C30-N4 & $1.35(2)$ & $\mathrm{C} 30-\mathrm{Pd} 2$ & $2.011(19)$ \\
\hline C31-N3 & $1.48(2)$ & C31-H31A & 0.98 \\
\hline C31-H31B & 0.98 & C31-H31C & 0.98 \\
\hline C32-C33 & 1.39 & C32-C37 & 1.39 \\
\hline C32-N3 & $1.392(17)$ & C33-C34 & 1.39 \\
\hline C33-H33 & 0.95 & C34-C35 & 1.39 \\
\hline C34-H34 & 0.95 & C $35-C 36$ & 1.39 \\
\hline
\end{tabular}




\begin{tabular}{|c|c|c|c|}
\hline C35-H35 & 0.95 & C36-C37 & 1.39 \\
\hline C36-H36 & 0.95 & C37-N4 & $1.389(16)$ \\
\hline C38-N4 & $1.45(2)$ & C38-C39 & $1.51(3)$ \\
\hline C38-P2 & $1.85(2)$ & C38-H38 & 1.0 \\
\hline C39-C40 & $1.52(2)$ & C39-H39A & 0.99 \\
\hline C39-H39B & 0.99 & $\mathrm{C} 40-\mathrm{O} 2$ & $1.24(2)$ \\
\hline C40-C41 & $1.51(2)$ & $\mathrm{C} 41-\mathrm{C} 42$ & 1.39 \\
\hline C41-C46 & 1.39 & C42-C43 & 1.39 \\
\hline C42-H42 & 0.95 & C43-C44 & 1.39 \\
\hline C43-H43 & 0.95 & C44-C45 & 1.39 \\
\hline C44-H44 & 0.95 & $\mathrm{C} 45-\mathrm{C} 46$ & 1.39 \\
\hline C45-H45 & 0.95 & C46-H46 & 0.95 \\
\hline C47-C48 & 1.39 & C47-C52 & 1.39 \\
\hline C47-P2 & $1.824(9)$ & C48-C49 & 1.39 \\
\hline C48-H48 & 0.95 & C49-C50 & 1.39 \\
\hline C49-H49 & 0.95 & C50-C51 & 1.39 \\
\hline C50-H50 & 0.95 & C51-C52 & 1.39 \\
\hline C51-H51 & 0.95 & C52-H52 & 0.95 \\
\hline C53-C54 & 1.39 & C53-C58 & 1.39 \\
\hline C53-P2 & $1.804(16)$ & C54-C55 & 1.39 \\
\hline C54-H54 & 0.95 & C55-C56 & 1.39 \\
\hline C55-H55 & 0.95 & C56-C57 & 1.39 \\
\hline C56-H56 & 0.95 & C57-C58 & 1.39 \\
\hline C57-H57 & 0.95 & C58-H58 & 0.95 \\
\hline $\begin{array}{l}\text { C53A- } \\
\text { C54A }\end{array}$ & $1.35(5)$ & $\begin{array}{l}\text { C53A- } \\
\text { C58A }\end{array}$ & $1.42(4)$ \\
\hline C53A-P2 & $1.795(17)$ & $\begin{array}{l}\text { C54A- } \\
\text { C55A }\end{array}$ & $1.40(5)$ \\
\hline $\begin{array}{l}\text { C54A- } \\
\text { H54A }\end{array}$ & 0.95 & $\begin{array}{l}\text { C55A- } \\
\text { C56A }\end{array}$ & $1.32(5)$ \\
\hline $\begin{array}{l}\text { C55A- } \\
\text { H55A }\end{array}$ & 0.95 & $\begin{array}{l}\text { C56A- } \\
\text { C57A }\end{array}$ & $1.33(5)$ \\
\hline $\begin{array}{l}\text { C56A- } \\
\text { H56A }\end{array}$ & 0.95 & $\begin{array}{l}\text { C57A- } \\
\text { C58A }\end{array}$ & $1.45(5)$ \\
\hline $\begin{array}{l}\text { C57A- } \\
\text { H57A }\end{array}$ & 0.95 & $\begin{array}{l}\text { C58A- } \\
\text { H58A }\end{array}$ & 0.95 \\
\hline C59-N5 & $1.33(2)$ & C59-N6 & $1.36(2)$ \\
\hline C59-Pd3 & $2.023(17)$ & C60-N5 & $1.44(2)$ \\
\hline C60-H60A & 0.98 & C60-H60B & 0.98 \\
\hline C60-H60C & 0.98 & C61-N5 & $1.371(16)$ \\
\hline C61-C62 & 1.39 & C61-C66 & 1.39 \\
\hline C62-C63 & 1.39 & C62-H62 & 0.95 \\
\hline
\end{tabular}




\begin{tabular}{|c|c|c|c|}
\hline C63-C64 & 1.39 & C63-H63 & 0.95 \\
\hline C64-C65 & 1.39 & C64-H64 & 0.95 \\
\hline C65-C66 & 1.39 & C65-H65 & 0.95 \\
\hline C66-N6 & $1.375(17)$ & C67-N6 & $1.46(2)$ \\
\hline C67-C68 & $1.51(3)$ & C67-P3 & $1.87(2)$ \\
\hline C67-H67 & 1.0 & C68-C69 & $1.53(3)$ \\
\hline C68-H68A & 0.99 & C68-H68B & 0.99 \\
\hline C69-O3 & $1.20(2)$ & C69-C70 & $1.46(2)$ \\
\hline C70-C71 & 1.39 & C70-C75 & 1.39 \\
\hline C71-C72 & 1.39 & $\mathrm{C} 71-\mathrm{H} 71$ & 0.95 \\
\hline C72-C73 & 1.39 & C72-H72 & 0.95 \\
\hline C73-C74 & 1.39 & C73-H73 & 0.95 \\
\hline C74-C75 & 1.39 & C74-H74 & 0.95 \\
\hline C75-H75 & 0.95 & C76-C77 & 1.39 \\
\hline C76-C81 & 1.39 & C76-P3 & $1.815(14)$ \\
\hline C77-C78 & 1.39 & C77-H77 & 0.95 \\
\hline C78-C79 & 1.39 & C78-H78 & 0.95 \\
\hline C79-C80 & 1.39 & C79-H79 & 0.95 \\
\hline C80-C81 & 1.39 & C80-H80 & 0.95 \\
\hline C81-H81 & 0.95 & $\begin{array}{l}\text { C76A- } \\
\text { C77A }\end{array}$ & 1.39 \\
\hline $\begin{array}{l}\text { C76A- } \\
\text { C81A }\end{array}$ & 1.39 & C76A-P3 & $1.785(15)$ \\
\hline $\begin{array}{l}\text { C77A- } \\
\text { C78A }\end{array}$ & 1.39 & $\begin{array}{l}\text { C77A- } \\
\text { H77A }\end{array}$ & 0.95 \\
\hline $\begin{array}{l}\text { C78A- } \\
\text { C79A }\end{array}$ & 1.39 & $\begin{array}{l}\text { C78A- } \\
\text { H78A }\end{array}$ & 0.95 \\
\hline $\begin{array}{l}\text { C79A- } \\
\text { C80A }\end{array}$ & 1.39 & $\begin{array}{l}\text { C79A- } \\
\text { H79A }\end{array}$ & 0.95 \\
\hline $\begin{array}{l}\text { C80A- } \\
\text { C81A }\end{array}$ & 1.39 & $\begin{array}{l}\text { C80A- } \\
\text { H80A }\end{array}$ & 0.95 \\
\hline $\begin{array}{l}\text { C81A- } \\
\text { H81A }\end{array}$ & 0.95 & C82-C83 & 1.39 \\
\hline C82-C87 & 1.39 & C82-P3 & $1.841(12)$ \\
\hline C83-C84 & 1.39 & C83-H83 & 0.95 \\
\hline C84-C85 & 1.39 & C84-H84 & 0.95 \\
\hline C85-C86 & 1.39 & C85-H85 & 0.95 \\
\hline C86-C87 & 1.39 & C86-H86 & 0.95 \\
\hline C87-H87 & 0.95 & $\begin{array}{l}\text { C82A- } \\
\text { C83A }\end{array}$ & 1.39 \\
\hline $\begin{array}{l}\text { C82A- } \\
\text { C87A }\end{array}$ & 1.39 & C82A-P3 & $1.757(15)$ \\
\hline
\end{tabular}




\begin{tabular}{|c|c|c|c|}
\hline $\begin{array}{l}\text { C83A- } \\
\text { C84A }\end{array}$ & 1.39 & $\begin{array}{l}\text { C83A- } \\
\text { H83A }\end{array}$ & 0.95 \\
\hline $\begin{array}{l}\text { C84A- } \\
\text { C85A }\end{array}$ & 1.39 & $\begin{array}{l}\text { C84A- } \\
\text { H84A }\end{array}$ & 0.95 \\
\hline $\begin{array}{l}\text { C85A- } \\
\text { C86A }\end{array}$ & 1.39 & $\begin{array}{l}\text { C85A- } \\
\text { H85A }\end{array}$ & 0.95 \\
\hline $\begin{array}{l}\text { C86A- } \\
\text { C87A }\end{array}$ & 1.39 & $\begin{array}{l}\text { C86A- } \\
\text { H86A }\end{array}$ & 0.95 \\
\hline $\begin{array}{l}\text { C87A- } \\
\text { H87A }\end{array}$ & 0.95 & Cl1-Pd1 & $2.368(4)$ \\
\hline $\mathrm{Cl2}-\mathrm{Pd} 1$ & $2.335(6)$ & $\mathrm{Cl} 3-\mathrm{Pd} 2$ & $2.364(4)$ \\
\hline $\mathrm{Cl} 4-\mathrm{Pd} 2$ & $2.335(5)$ & $\mathrm{C} 15-\mathrm{Pd} 3$ & $2.371(5)$ \\
\hline Cl6-Pd3 & $2.357(5)$ & P1-Pd1 & $2.206(4)$ \\
\hline $\mathrm{P} 2-\mathrm{Pd} 2$ & $2.203(5)$ & P3-Pd3 & $2.216(5)$ \\
\hline
\end{tabular}

$\begin{array}{llll}\text { N2-C1-N1 } & 103.7(17) & \text { N2-C1-Pd1 } & 122.1(15) \\ \text { N1-C1-Pd1 } & 132.8(14) & \text { N1-C2-H2A } & 109.5 \\ \text { N1-C2-H2B } & 109.5 & \text { H2A-C2-H2B } & 109.5 \\ \text { N1-C2-H2C } & 109.5 & \text { H2A-C2-H2C } & 109.5 \\ \text { H2B-C2-H2C } & 109.5 & \text { N1-C3-C4 } & 131.8(10) \\ \text { N1-C3-C8 } & 108.2(10) & \text { C4-C3-C8 } & 120.0 \\ \text { C3-C4-C5 } & 120.0 & \text { C3-C4-H4 } & 120.0 \\ \text { C5-C4-H4 } & 120.0 & \text { C4-C5-C6 } & 120.0 \\ \text { C4-C5-H5 } & 120.0 & \text { C6-C5-H5 } & 120.0 \\ \text { C7-C6-C5 } & 120.0 & \text { C7-C6-H6 } & 120.0 \\ \text { C5-C6-H6 } & 120.0 & \text { C6-C7-C8 } & 120.0 \\ \text { C6-C7-H7 } & 120.0 & \text { C8-C7-H7 } & 120.0 \\ \text { N2-C8-C7 } & 134.3(10) & \text { N2-C8-C3 } & 105.7(10) \\ \text { C7-C8-C3 } & 120.0 & \text { N2-C9-C10 } & 112.6(15) \\ \text { N2-C9-P1 } & 101.5(11) & \text { C10-C9-P1 } & 114.5(13) \\ \text { N2-C9-H9 } & 109.3 & \text { C10-C9-H9 } & 109.3 \\ \text { P1-C9-H9 } & 109.3 & \text { C11-C10-C9 } & 112.2(16) \\ \text { C11-C10-H10A } 109.2 & \text { C9-C10-H10A } & 109.2 \\ \text { C11-C10-H10B } & 109.2 & \text { C9-C10-H10B } & 109.2 \\ \text { H10A-C10- } & 107.9 & \text { O1-C11-C12 } & 122.5(17) \\ \text { H10B } & & & \\ \text { O1-C11-C10 } & 119.0(18) & \text { C12-C11-C10 } & 118.5(15) \\ \text { C13-C12-C17 } & 120.0 & \text { C13-C12-C11 } & 122.3(10) \\ \text { C17-C12-C11 } & 117.7(10) & \text { C14-C13-C12 } & 120.0 \\ \text { C14-C13-H13 } & 120.0 & \text { C12-C13-H13 } & 120.0\end{array}$




$\begin{array}{llll}\text { C13-C14-C15 } & 120.0 & \text { C13-C14-H14 } & 120.0 \\ \text { C15-C14-H14 } & 120.0 & \text { C14-C15-C16 } & 120.0 \\ \text { C14-C15-H15 } & 120.0 & \text { C16-C15-H15 } & 120.0 \\ \text { C17-C16-C15 } & 120.0 & \text { C17-C16-H16 } & 120.0 \\ \text { C15-C16-H16 } & 120.0 & \text { C16-C17-C12 } & 120.0 \\ \text { C16-C17-H17 } & 120.0 & \text { C12-C17-H17 } & 120.0 \\ \text { C19-C18-C23 } & 120.0 & \text { C19-C18-P1 } & 117.1(7) \\ \text { C23-C18-P1 } & 122.8(7) & \text { C20-C19-C18 } & 120.0 \\ \text { C20-C19-H19 } & 120.0 & \text { C18-C19-H19 } & 120.0 \\ \text { C21-C20-C19 } & 120.0 & \text { C21-C20-H20 } & 120.0 \\ \text { C19-C20-H20 } & 120.0 & \text { C20-C21-C22 } & 120.0 \\ \text { C20-C21-H21 } & 120.0 & \text { C22-C21-H21 } & 120.0 \\ \text { C21-C22-C23 } & 120.0 & \text { C21-C22-H22 } & 120.0 \\ \text { C23-C22-H22 } & 120.0 & \text { C22-C23-C18 } & 120.0 \\ \text { C22-C23-H23 } & 120.0 & \text { C18-C23-H23 } & 120.0 \\ \text { C25-C24-C29 } & 120.0 & \text { C25-C24-P1 } & 119.2(6) \\ \text { C29-C24-P1 } & 120.8(6) & \text { C24-C25-C26 } & 120.0 \\ \text { C24-C25-H25 } & 120.0 & \text { C26-C25-H25 } & 120.0 \\ \text { C27-C26-C25 } & 120.0 & \text { C27-C26-H26 } & 120.0 \\ \text { C25-C26-H26 } & 120.0 & \text { C26-C27-C28 } & 120.0 \\ \text { C26-C27-H27 } & 120.0 & \text { C28-C27-H27 } & 120.0 \\ \text { C29-C28-C27 } & 120.0 & \text { C29-C28-H28 } & 120.0 \\ \text { C27-C28-H28 } & 120.0 & \text { C28-C29-C24 } & 120.0 \\ \text { C28-C29-H29 } & 120.0 & \text { C24-C29-H29 } & 120.0 \\ \text { N3-C30-N4 } & 108.6(16) & \text { N3-C30-Pd2 } & 129.7(14) \\ \text { N4-C30-Pd2 } & 120.0(13) & \text { N3-C31-H31A } & 109.5 \\ \text { N3-C31-H31B } & 109.5 & \text { H31A-C31- } & 109.5 \\ & & \text { H31B } & \\ \text { N3-C31-H31C } & 109.5 & \text { H31A-C31- } & 109.5 \\ \text { H31B-C31- } & & \text { H31C } & \\ \text { H31C } & 109.5 & \text { C33-C32-C37 } & 120.0 \\ \text { C33-C32-N3 } & 132.9(10) & \text { C37-C32-N3 } & 107.1(10) \\ \text { C32-C33-C34 } & 120.0 & \text { C32-C33-H33 } & 120.0 \\ \text { C34-C33-H33 } & 120.0 & \text { C35-C34-C33 } & 120.0 \\ \text { C35-C34-H34 } & 120.0 & \text { C33-C34-H34 } & 120.0 \\ \text { C36-C35-C34 } & 120.0 & \text { C36-C35-H35 } & 120.0 \\ \text { C34-C35-H35 } & 120.0 & \text { C35-C36-C37 } & 120.0 \\ \text { C35-C36-H36 } & 120.0 & \text { C37-C36-H36 } & 120.0 \\ \text { N4-C37-C36 } & 133.9(9) & \text { N4-C37-C32 } & 105.9(9) \\ \text { C36-C37-C32 } & 120.0 & \text { N4-C38-C39 } & 112.4(16) \\ \text { N4-C38-P2 } & 102.6(12) & \text { C39-C38-P2 } & 114.3(13) \\ & & & \\ & & \end{array}$




\begin{tabular}{|c|c|c|c|}
\hline N4-C38-H38 & 109.1 & C39-C38-H38 & 109.1 \\
\hline P2-C38-H38 & 109.1 & C38-C39-C40 & $112.1(16)$ \\
\hline C38-C39-H39A & 109.2 & C40-C39-H39A & 109.2 \\
\hline C38-C39-H39B & 109.2 & С40-С39-Н39B & 109.2 \\
\hline $\begin{array}{l}\text { H39A-C39- } \\
\text { H39B }\end{array}$ & 107.9 & $\mathrm{O} 2-\mathrm{C} 40-\mathrm{C} 41$ & 119.7(16) \\
\hline O2-C40-C39 & $119.0(18)$ & C41-C40-C39 & $121.2(15)$ \\
\hline C42-C41-C46 & 120.0 & $\mathrm{C} 42-\mathrm{C} 41-\mathrm{C} 40$ & $120.2(11)$ \\
\hline C46-C41-C40 & $119.8(11)$ & C43-C42-C41 & 120.0 \\
\hline C43-C42-H42 & 120.0 & $\mathrm{C} 41-\mathrm{C} 42-\mathrm{H} 42$ & 120.0 \\
\hline C42-C43-C44 & 120.0 & $\mathrm{C} 42-\mathrm{C} 43-\mathrm{H} 43$ & 120.0 \\
\hline C44-C43-H43 & 120.0 & $\mathrm{C} 45-\mathrm{C} 44-\mathrm{C} 43$ & 120.0 \\
\hline C45-C44-H44 & 120.0 & C43-C44-H44 & 120.0 \\
\hline C46-C45-C44 & 120.0 & $\mathrm{C} 46-\mathrm{C} 45-\mathrm{H} 45$ & 120.0 \\
\hline C44-C45-H45 & 120.0 & $\mathrm{C} 45-\mathrm{C} 46-\mathrm{C} 41$ & 120.0 \\
\hline C45-C46-H46 & 120.0 & $\mathrm{C} 41-\mathrm{C} 46-\mathrm{H} 46$ & 120.0 \\
\hline C48-C47-C52 & 120.0 & C48-C47-P2 & $123.6(6)$ \\
\hline C52-C47-P2 & $116.2(6)$ & C47-C48-C49 & 120.0 \\
\hline C47-C48-H48 & 120.0 & C49-C48-H48 & 120.0 \\
\hline C50-C49-C48 & 120.0 & C50-C49-H49 & 120.0 \\
\hline C48-C49-H49 & 120.0 & C49-C50-C51 & 120.0 \\
\hline C49-C50-H50 & 120.0 & C51-C50-H50 & 120.0 \\
\hline C52-C51-C50 & 120.0 & C52-C51-H51 & 120.0 \\
\hline C50-C51-H51 & 120.0 & C51-C52-C47 & 120.0 \\
\hline C51-C52-H52 & 120.0 & C47-C52-H52 & 120.0 \\
\hline C54-C53-C58 & 120.0 & C54-C53-P2 & 119.(3) \\
\hline C58-C53-P2 & 121.(3) & C53-C54-C55 & 120.0 \\
\hline C53-C54-H54 & 120.0 & C55-C54-H54 & 120.0 \\
\hline C56-C55-C54 & 120.0 & C56-C55-H55 & 120.0 \\
\hline C54-C55-H55 & 120.0 & C55-C56-C57 & 120.0 \\
\hline C55-C56-H56 & 120.0 & C57-C56-H56 & 120.0 \\
\hline C58-C57-C56 & 120.0 & C58-C57-H57 & 120.0 \\
\hline C56-C57-H57 & 120.0 & C57-C58-C53 & 120.0 \\
\hline C57-C58-H58 & 120.0 & C53-C58-H58 & 120.0 \\
\hline $\begin{array}{l}\text { C54A-C53A- } \\
\text { C58A }\end{array}$ & 118.(3) & C54A-C53A-P2 & $119 .(4)$ \\
\hline C58A-C53A-P & 122.(4) & $\begin{array}{l}\text { C53A-C54A- } \\
\text { C55A }\end{array}$ & 122.(3) \\
\hline $\begin{array}{l}\text { C53A-C54A- } \\
\text { H54A }\end{array}$ & 119.2 & $\begin{array}{l}\text { C55A-C54A- } \\
\text { H54A }\end{array}$ & 119.2 \\
\hline $\begin{array}{l}\text { C56A-C55A- } \\
\text { C54A }\end{array}$ & 119.(3) & $\begin{array}{l}\text { C56A-C55A- } \\
\text { H55A }\end{array}$ & 120.5 \\
\hline
\end{tabular}




\begin{tabular}{|c|c|c|c|}
\hline $\begin{array}{l}\text { C54A-C55A- } \\
\text { H55A }\end{array}$ & 120.5 & $\begin{array}{l}\text { C55A-C56A- } \\
\text { C57A }\end{array}$ & 124.(4) \\
\hline $\begin{array}{l}\text { C55A-C56A- } \\
\text { H56A }\end{array}$ & 117.9 & $\begin{array}{l}\text { C57A-C56A- } \\
\text { H56A }\end{array}$ & 117.9 \\
\hline $\begin{array}{l}\text { C56A-C57A- } \\
\text { C58A }\end{array}$ & 118.(3) & $\begin{array}{l}\text { C56A-C57A- } \\
\text { H57A }\end{array}$ & 121.1 \\
\hline $\begin{array}{l}\text { C58A-C57A- } \\
\text { H57A }\end{array}$ & 121.1 & $\begin{array}{l}\text { C53A-C58A- } \\
\text { C57A }\end{array}$ & 119.(3) \\
\hline $\begin{array}{l}\text { C53A-C58A- } \\
\text { H58A }\end{array}$ & 120.7 & $\begin{array}{l}\text { C57A-C58A- } \\
\text { H58A }\end{array}$ & 120.7 \\
\hline N5-C59-N6 & $107.6(15)$ & N5-C59-Pd3 & $132.0(13)$ \\
\hline N6-C59-Pd3 & $118.6(13)$ & N5-C60-H60A & 109.5 \\
\hline N5-C60-H60B & 109.5 & $\begin{array}{l}\text { H60A-C60- } \\
\text { H60B }\end{array}$ & 109.5 \\
\hline N5-C60-H60C & 109.5 & $\begin{array}{l}\text { H60A-C60- } \\
\text { H60C }\end{array}$ & 109.5 \\
\hline $\begin{array}{l}\text { H60B-C60- } \\
\text { H60C }\end{array}$ & 109.5 & N5-C61-C62 & $132.8(10)$ \\
\hline N5-C61-C66 & $107.2(10)$ & C62-C61-C66 & 120.0 \\
\hline C61-C62-C63 & 120.0 & C61-C62-H62 & 120.0 \\
\hline C63-C62-H62 & 120.0 & C64-C63-C62 & 120.0 \\
\hline C64-C63-H63 & 120.0 & C62-C63-H63 & 120.0 \\
\hline C63-C64-C65 & 120.0 & C63-C64-H64 & 120.0 \\
\hline C65-C64-H64 & 120.0 & C64-C65-C66 & 120.0 \\
\hline C64-C65-H65 & 120.0 & C66-C65-H65 & 120.0 \\
\hline N6-C66-C65 & $134.3(10)$ & N6-C66-C61 & $105.7(10)$ \\
\hline C65-C66-C61 & 120.0 & N6-C67-C68 & $111.0(16)$ \\
\hline N6-C67-P3 & $102.6(12)$ & C68-C67-P3 & $115.7(15)$ \\
\hline N6-C67-H67 & 109.1 & C68-C67-H67 & 109.1 \\
\hline P3-C67-H67 & 109.1 & C67-C68-C69 & $112.5(18)$ \\
\hline C67-C68-H68A & 109.1 & C69-C68-H68A & 109.1 \\
\hline C67-C68-H68B & 109.1 & C69-C68-H68B & 109.1 \\
\hline $\begin{array}{l}\text { H68A-C68- } \\
\text { H68B }\end{array}$ & 107.8 & O3-C69-C70 & $124.1(18)$ \\
\hline O3-C69-C68 & 118.(2) & C70-C69-C68 & $118.1(17)$ \\
\hline C71-C70-C75 & 120.0 & C71-C70-C69 & $116.0(11)$ \\
\hline C75-C70-C69 & $124.0(11)$ & C70-C71-C72 & 120.0 \\
\hline C70-C71-H71 & 120.0 & C72-C71-H71 & 120.0 \\
\hline C71-C72-C73 & 120.0 & C71-C72-H72 & 120.0 \\
\hline C73-C72-H72 & 120.0 & C74-C73-C72 & 120.0 \\
\hline C74-C73-H73 & 120.0 & C72-C73-H73 & 120.0 \\
\hline C75-C74-C73 & 120.0 & C75-C74-H74 & 120.0 \\
\hline C73-C74-H74 & 120.0 & C74-C75-C70 & 120.0 \\
\hline
\end{tabular}




\begin{tabular}{|c|c|c|c|}
\hline C74-C75-H75 & 120.0 & C70-C75-H75 & 120.0 \\
\hline C77-C76-C81 & 120.0 & C77-C76-P3 & $121.3(16)$ \\
\hline C81-C76-P3 & 118.7(16) & C76-C77-C78 & 120.0 \\
\hline C76-C77-H77 & 120.0 & C78-C77-H77 & 120.0 \\
\hline C79-C78-C77 & 120.0 & C79-C78-H78 & 120.0 \\
\hline C77-C78-H78 & 120.0 & C78-C79-C80 & 120.0 \\
\hline C78-C79-H79 & 120.0 & C80-C79-H79 & 120.0 \\
\hline C81-C80-C79 & 120.0 & C81-C80-H80 & 120.0 \\
\hline C79-C80-H80 & 120.0 & C80-C81-C76 & 120.0 \\
\hline C80-C81-H81 & 120.0 & C76-C81-H81 & 120.0 \\
\hline $\begin{array}{l}\text { C77A-C76A- } \\
\text { C81A }\end{array}$ & 120.0 & C77A-C76A-P3 & $125.2(18)$ \\
\hline C81A-C76A-P3 & $114.1(18)$ & $\begin{array}{l}\text { C76A-C77A- } \\
\text { C78A }\end{array}$ & 120.0 \\
\hline $\begin{array}{l}\text { C76A-C77A- } \\
\text { H77A }\end{array}$ & 120.0 & $\begin{array}{l}\text { C78A-C77A- } \\
\text { H77A }\end{array}$ & 120.0 \\
\hline $\begin{array}{l}\text { C77A-C78A- } \\
\text { C79A }\end{array}$ & 120.0 & $\begin{array}{l}\text { C77A-C78A- } \\
\text { H78A }\end{array}$ & 120.0 \\
\hline $\begin{array}{l}\text { C79A-C78A- } \\
\text { H78A }\end{array}$ & 120.0 & $\begin{array}{l}\text { C80A-C79A- } \\
\text { C78A }\end{array}$ & 120.0 \\
\hline $\begin{array}{l}\text { C80A-C79A- } \\
\text { H79A }\end{array}$ & 120.0 & $\begin{array}{l}\text { C78A-C79A- } \\
\text { H79A }\end{array}$ & 120.0 \\
\hline $\begin{array}{l}\text { C79A-C80A- } \\
\text { C81A }\end{array}$ & 120.0 & $\begin{array}{l}\text { C79A-C80A- } \\
\text { H80A }\end{array}$ & 120.0 \\
\hline $\begin{array}{l}\text { C81A-C80A- } \\
\text { H80A }\end{array}$ & 120.0 & $\begin{array}{l}\text { C80A-C81A- } \\
\text { C76A }\end{array}$ & 120.0 \\
\hline $\begin{array}{l}\text { C80A-C81A- } \\
\text { H81A }\end{array}$ & 120.0 & $\begin{array}{l}\text { C76A-C81A- } \\
\text { H81A }\end{array}$ & 120.0 \\
\hline C83-C82-C87 & 120.0 & C83-C82-P3 & $118.4(12)$ \\
\hline C87-C82-P3 & $121.6(12)$ & $\mathrm{C} 84-\mathrm{C} 83-\mathrm{C} 82$ & 120.0 \\
\hline C84-C83-H83 & 120.0 & $\mathrm{C} 82-\mathrm{C} 83-\mathrm{H} 83$ & 120.0 \\
\hline C83-C84-C85 & 120.0 & C83-C84-H84 & 120.0 \\
\hline C85-C84-H84 & 120.0 & C86-C85-C84 & 120.0 \\
\hline C86-C85-H85 & 120.0 & C84-C85-H85 & 120.0 \\
\hline C85-C86-C87 & 120.0 & C85-C86-H86 & 120.0 \\
\hline C87-C86-H86 & 120.0 & C86-C87-C82 & 120.0 \\
\hline C86-C87-H87 & 120.0 & C82-C87-H87 & 120.0 \\
\hline $\begin{array}{l}\text { C83A-C82A- } \\
\text { C87A }\end{array}$ & 120.0 & C83A-C82A-P & $115 .(2)$ \\
\hline C87A-C82A-P3 & $125 .(2)$ & $\begin{array}{l}\text { C84A-C83A- } \\
\text { C82A }\end{array}$ & 120.0 \\
\hline $\begin{array}{l}\text { C84A-C83A- } \\
\text { H83A }\end{array}$ & 120.0 & $\begin{array}{l}\text { C82A-C83A- } \\
\text { H83A }\end{array}$ & 120.0 \\
\hline
\end{tabular}




\begin{tabular}{|c|c|c|c|}
\hline $\begin{array}{l}\text { C83A-C } \\
\text { C85A }\end{array}$ & 120.0 & $\begin{array}{l}\text { C83A-C84A- } \\
\text { H84A }\end{array}$ & 120.0 \\
\hline $\begin{array}{l}\text { C85A-C84A- } \\
\text { H84A }\end{array}$ & 120.0 & $\begin{array}{l}\text { C86A-C85A- } \\
\text { C84A }\end{array}$ & 120.0 \\
\hline $\begin{array}{l}\text { C86A-C85A- } \\
\text { H85A }\end{array}$ & 120.0 & $\begin{array}{l}\text { C84A-C85A- } \\
\text { H85A }\end{array}$ & 120.0 \\
\hline $\begin{array}{l}\text { C87A-C86A- } \\
\text { C85A }\end{array}$ & 120.0 & $\begin{array}{l}\text { C87A-C86A- } \\
\text { H86A }\end{array}$ & 120.0 \\
\hline $\begin{array}{l}\text { C85A-C86A- } \\
\text { H86A }\end{array}$ & 120.0 & $\begin{array}{l}\text { C86A-C87A- } \\
\text { C82A }\end{array}$ & 120.0 \\
\hline $\begin{array}{l}\text { C86A-C87A- } \\
\text { H87A }\end{array}$ & 120.0 & $\begin{array}{l}\text { C82A-C87A- } \\
\text { H87A }\end{array}$ & 120.0 \\
\hline C3-N1-C1 & $110.2(14)$ & $\mathrm{C} 3-\mathrm{N} 1-\mathrm{C} 2$ & $120.2(15)$ \\
\hline C1-N1-C2 & 129.6(16) & C8-1 & $112.1(15)$ \\
\hline C8-N2-C9 & $125.8(13)$ & C1-N2-C9 & $122.0(16)$ \\
\hline C30-N3-C32 & $108.8(14)$ & C30-N3-C31 & $130.9(16)$ \\
\hline $\mathrm{C} 32-\mathrm{N}$ & $120.3(14)$ & $\mathrm{C} 30-$ & (13) \\
\hline C30-N4-C38 & $123.3(15)$ & C37-N4-C38 & $127.0(14)$ \\
\hline C59-N5-C61 & $109.8(13)$ & C59-N5-C60 & $128.5(16)$ \\
\hline C61-N & $121.6(15)$ & C59-N6-C66 & $109.6(14)$ \\
\hline C59-N6-C67 & $124.6(16)$ & C66-N6-C67 & $125.3(14)$ \\
\hline C18-P1-C24 & $109.5(6)$ & C18-P1-C9 & $107.1(7)$ \\
\hline C24-P1-C9 & $103.8(8)$ & C18-P1-Pd1 & $113.6(4)$ \\
\hline C24-P1-Pd1 & $117.2(4)$ & C9-P1-Pd1 & $104.6(6)$ \\
\hline C53A-P2-C47 & 113.(2) & C53-P2-C47 & 109.(2) \\
\hline C53A-P2-C38 & 103.(2) & C53-P2-C38 & 101.(2) \\
\hline C47-P2-C38 & $108.4(7)$ & C53A-P2-Pd2 & 114.(2) \\
\hline C53-P2-Pd2 & 119.(2) & C47-P2-Pd2 & $114.0(4)$ \\
\hline C38-P2-Pd2 & $103.9(6)$ & C82A-P3-C76A & $104.1(16)$ \\
\hline C76-P3-C82 & $115.2(13)$ & C82A-P3-C67 & $105.6(14)$ \\
\hline C76A-P3-C67 & $108.9(12)$ & C76-P3-C67 & $105.6(11)$ \\
\hline C82-P3-C67 & $101.2(10)$ & C82A-P3-Pd3 & $116.6(12)$ \\
\hline C76A-P3-Pd3 & $117.3(11)$ & C76-P3-Pd3 & $112.9(10)$ \\
\hline C82-P3-Pd3 & $116.2(7)$ & C67-P3-Pd3 & $103.6(6)$ \\
\hline C1-Pd1-P1 & $82.0(6)$ & $\mathrm{C} 1-\mathrm{Pd} 1-\mathrm{Cl} 2$ & $166.7(5)$ \\
\hline $\mathrm{P} 1-\mathrm{Pd} 1-\mathrm{Cl} 2$ & $88.73(18)$ & C1-Pd1-Cl1 & $100.2(6)$ \\
\hline P1-Pd1-Cl1 & $170.32(18)$ & $\mathrm{Cl} 2-\mathrm{Pd} 1-\mathrm{Cl} 1$ & $90.53(18)$ \\
\hline $\mathrm{C} 30-\mathrm{Pd} 2-\mathrm{P} 2$ & $81.8(5)$ & $\mathrm{C} 30-\mathrm{Pd} 2-\mathrm{Cl} 4$ & $164.3(6)$ \\
\hline $\mathrm{P} 2-\mathrm{Pd} 2-\mathrm{Cl} 4$ & $87.27(18)$ & $\mathrm{C} 30-\mathrm{Pd} 2-\mathrm{Cl} 3$ & $101.0(5)$ \\
\hline $\mathrm{P} 2-\mathrm{Pd} 2-\mathrm{Cl} 3$ & $172.18(19)$ & $\mathrm{Cl} 4-\mathrm{Pd} 2-\mathrm{Cl} 3$ & $91.38(18)$ \\
\hline C59-Pd3-P3 & $82.7(5)$ & C59-Pd3-Cl6 & $166.9(6)$ \\
\hline P3-Pd3-Cl6 & $89.38(19)$ & C59-Pd3-Cl5 & $98.8(5)$ \\
\hline
\end{tabular}


P3-Pd3-Cl5 171.3(2) Cl6-Pd3-Cl5 90.55(17) 
Complete optimizations of 1a using $(R)-C 3$<smiles>O=C(/C=C/n1ccnc1)c1ccccc1</smiles>

$1 \mathrm{a}$
1. (R)-C3, $\mathrm{HPPh}_{2}$, base, solvent, $T, 8 \mathrm{~h}$ 2. $\mathrm{H}_{2} \mathrm{O}_{2}(\mathrm{aq})$, r.t., 5 min<smiles>CCOPC(CC(=O)c1ccccc1)n1ccnc1</smiles>

$(S)-3 a$

Table S1: Optimization $(S)-\mathbf{3 a}$

\begin{tabular}{|c|c|c|c|c|c|c|c|c|}
\hline entry $^{[a]}$ & base & $\begin{array}{c}\text { base } \\
\text { loading/ } \\
\text { equiv. }\end{array}$ & $\begin{array}{l}\text { HPPh2 } \\
\text { loading/ } \\
\text { equiv. }\end{array}$ & solvent & $\begin{array}{l}\mathbf{T} / \\
{ }^{\circ} \mathbf{C}\end{array}$ & $\begin{array}{c}(R)-C 3 \\
\text { loading/ } \\
\text { mol\% }\end{array}$ & $\operatorname{conv}^{[b]} / \%$ & $\mathrm{ee}^{[\mathrm{c}] / \%}$ \\
\hline 1 & NEt3 & 1 & 1 & THF & r.t. & 5 & $>99$ & 78 \\
\hline 2 & $\mathrm{NEt}_{3}$ & 1 & 1 & $\mathrm{CH}_{2} \mathrm{Cl}_{2}$ & r.t. & 5 & $>99$ & 80 \\
\hline 3 & $\mathrm{NEt}_{3}$ & 1 & 1 & $\mathrm{Me}_{2} \mathrm{CO}$ & r.t. & 5 & $>99$ & 51 \\
\hline 4 & $\mathrm{NEt}_{3}$ & 1 & 1 & NCMe & r.t. & 5 & $>99$ & 65 \\
\hline 5 & $\mathrm{NEt}_{3}$ & 1 & 1 & $\mathrm{EtOH}$ & r.t. & 5 & $>99$ & 13 \\
\hline 6 & $\mathrm{NEt}_{3}$ & 1 & 1 & $\mathrm{CHCl}_{3}$ & r.t. & 5 & $>99$ & 75 \\
\hline 7 & $\mathrm{NEt}_{3}$ & 1 & 1 & EtOAc & r.t. & 5 & $>99$ & 59 \\
\hline 8 & ${ }^{i} \operatorname{Pr}_{2} \mathrm{NH}$ & 1 & 1 & $\mathrm{CH}_{2} \mathrm{Cl}_{2}$ & r.t. & 5 & $>99$ & 51 \\
\hline 9 & TMEDA & 1 & 1 & $\mathrm{CH}_{2} \mathrm{Cl}_{2}$ & r.t. & 5 & $>99$ & 82 \\
\hline 10 & DBU & 1 & 1 & $\mathrm{CH}_{2} \mathrm{Cl}_{2}$ & r.t. & 5 & $>99$ & 2 \\
\hline 11 & pyridine & 1 & 1 & $\mathrm{CH}_{2} \mathrm{Cl}_{2}$ & r.t. & 5 & $>99$ & n.d. ${ }^{[d]}$ \\
\hline 12 & $\mathrm{NaOAc}$ & 1 & 1 & $\mathrm{CH}_{2} \mathrm{Cl}_{2}$ & r.t. & 5 & $>99$ & 73 \\
\hline 13 & $\mathrm{Na}_{2} \mathrm{CO}_{3}$ & 1 & 1 & $\mathrm{CH}_{2} \mathrm{Cl}_{2}$ & r.t. & 5 & $>99$ & 69 \\
\hline 14 & TMEDA & 1 & 1 & $\mathrm{CH}_{2} \mathrm{Cl}_{2}$ & 0 & 5 & $>99$ & 92 \\
\hline 15 & TMEDA & 1 & 1 & $\mathrm{CH}_{2} \mathrm{Cl}_{2}$ & -40 & 5 & $>99$ & 93 \\
\hline 16 & TMEDA & 1 & 1 & $\mathrm{CH}_{2} \mathrm{Cl}_{2}$ & -78 & 5 & $>99$ & 96 \\
\hline 17 & TMEDA & 1 & 1 & $\mathrm{CH}_{2} \mathrm{Cl}_{2}$ & -78 & 10 & $>99$ & 96 \\
\hline 18 & TMEDA & 1 & 1 & $\mathrm{CH}_{2} \mathrm{Cl}_{2}$ & -78 & 2.5 & $>99$ & 96 \\
\hline 19 & TMEDA & 1 & 1 & $\mathrm{CH}_{2} \mathrm{Cl}_{2}$ & -78 & 1 & 40 & 96 \\
\hline 20 & TMEDA & 2 & 1 & $\mathrm{CH}_{2} \mathrm{Cl}_{2}$ & -78 & 2.5 & $>99$ & 96 \\
\hline 21 & TMEDA & 1 & 1 & $\mathrm{CH}_{2} \mathrm{Cl}_{2}$ & -78 & - & 0 & n.d. ${ }^{\mathrm{e}}$ \\
\hline
\end{tabular}

[a] Reactions were carried out using vinyl azole $1 \mathrm{a}(0.0575 \mathrm{mmol}, 1$ equiv.) with the stated equivalence of diphenylphosphine, $(R)$-C3 and respective base in the listed solvent (575 uL) at stated temperature for $8 \mathrm{~h}$ before being oxidized with one drop of $30 \% \mathrm{w} / \mathrm{w}$ aqueous hydrogen peroxide at the stated temperature.

${ }^{[b]}$ Obtained via integration of $30.2 \mathrm{ppm}$ from ${ }^{31} \mathrm{P}\left\{{ }^{1} \mathrm{H}\right\}$ NMR spectrum of crude product mixture.

${ }^{[c]}$ Determined by chiral HPLC.

[d] Product 3a was not formed. Product could not be isolated.

${ }^{[\mathrm{e}]}$ No reaction. 
1. Hoffmann, S.; Hartung, K.-J.; Hanh, N. T.; Mewes, R.; Baluzow, W., Synthesen vinyloger N-Acyl-azole (Vinazolide). Z. Anorg. Allg. Chem. 1986, 26 (3), 105-106. 